\title{
Spin-5 Casimir operator its three-point functions with two scalars
}

\author{
Changhyun Ahn and Hyunsu Kim \\ Dept. of Physics, Kyungpook National University, \\ Tae-Hak-Ro 80, Buk-Gu, Taegu, 702-701, South Korea \\ E-mail: ahn@knu.ac.kr, kimhyun@knu.ac.kr
}

ABSTRACT: By calculating the second-order pole in the operator product expansion (OPE) between the spin-3 Casimir operator and the spin-4 Casimir operator known previously, the spin-5 Casimir operator is obtained in the coset model based on $\left(A_{N-1}^{(1)} \oplus A_{N-1}^{(1)}, A_{N-1}^{(1)}\right)$ at level $(k, 1)$. This spin-5 Casimir operator consisted of the quintic, quartic (with one derivative) and cubic (with two derivatives) WZW currents contracted with $\mathrm{SU}(N)$ invariant tensors. The three-point functions with two scalars for all values of 't Hooft coupling in the large $N$ limit were obtained by analyzing the zero-mode eigenvalue equations carefully. These three-point functions were dual to those in $A d S_{3}$ higher spin gravity theory with matter. Furthermore, the exact three-point functions that hold for any finite $N$ and $k$ are obtained. The zero mode eigenvalue equations for the spin-5 current in CFT coincided with those of the spin- 5 field in asymptotic symmetry algebra of the higher spin theory on the $A d S_{3}$. This paper also describes the structure constant appearing in the spin- 4 Casimir operator from the OPE between the spin-3 Casimir operator and itself for $N=4,5$ in the more general coset minimal model with two arbitrary levels $\left(k_{1}, k_{2}\right)$.

Keywords: AdS-CFT Correspondence, Conformal and W Symmetry

ARXIV EPRINT: 1308.1726 


\section{Contents}

1 Introduction 1

2 Generalization of the GKO coset construction: review 4

3 Spin-5 Casimir operator $\quad 6$

3.1 Review on the spin-3 and spin-4 currents 6

$\begin{array}{lll}3.2 & \text { The spin-5 current } & 7\end{array}$

4 Three-point functions $\quad \mathbf{1 0}$

4.1 Eigenvalue equation of the zero mode of spin-5 current acting on the state $|(f ; 0)\rangle$ in the large $N$ limit $\quad 10$

$\begin{array}{ll}\text { 4.1.1 Eigenvalue equation } & 10\end{array}$

4.2 Eigenvalue equation of the zero mode of the spin-5 current acting on the state $|(0 ; f)\rangle$ in the large $N$ limit 13

$\begin{array}{lll}4.3 & \text { Three-point functions in the large } N \text { limit } & 15\end{array}$

4.4 Eigenvalue equations and three-point functions at a finite $(N, k) \quad 17$

$\begin{array}{ll}4.5 & \text { More general coset model } \\ 20\end{array}$

5 Conclusions and outlook $\quad 21$

$\begin{array}{ll}\text { A Coefficient functions appearing in the spin-4 current } & 23\end{array}$

B Intermediate spin-5 field contents in the second-order pole of the OPE $\begin{array}{ll}W^{(3)}(z) W^{(4)}(w) & 26\end{array}$

C Details of the fully normal ordered spin-5 operator 30

D Explicit 52 coefficient functions of spin-5 Casimir operator 33

E Eigenvalue equations for the spin-5 current on the perturbative state 43

F Eigenvalue equations for other higher spin currents of spins $s=2,3,4 \quad 47$

\section{Introduction}

Simple examples of the AdS/CFT correspondence allow a detailed study of holography that would not be possible in a string theory setup. Recent progress along these lines is based on an examination of higher spin theories of gravity in AdS space, which include a large number of fields with spins $s=2,3, \cdots, N$. In three bulk dimensions, the higher spin theory is quite simple. The massless sector is given semiclassically by the Chern-Simons 
action. Therefore, the graviton and its higher spin fields have no propagating modes. Twodimensional conformal field theories are well-established quantum field theories and have a range of applications in different areas of physics. A high degree of analytical control over these CFTs can provide a rich source of CFTs with nontrivial bulk AdS duals. The simple theory is described using large $N$ vector models. This will give rise to a controlled environment in which to study the puzzle of the emergence of a gravity dual.

The $W_{N}$ minimal model conformal field theory (CFT) is dual in the 't Hooft $\frac{1}{N}$ expansion to the higher spin theory of Vasiliev on the $A d S_{3}$ coupled with one complex scalar $[1,2]$. The CFTs have the following diagonal coset, $\frac{G}{H}$, which is expressed as

$$
\frac{G}{H}=\frac{\widehat{S U}(N)_{k} \oplus \widehat{S U}(N)_{1}}{\widehat{S U}(N)_{k+1}} .
$$

The higher spin-s Casimir currents of degree $s$ with $s=3, \cdots, N$ can be obtained from polynomial combinations of the individual numerator $\mathrm{SU}(N)$ currents of spin-1 [3]. The diagonal denominator $\mathrm{SU}(N)$ currents of spin-1 commute with these higher spin- $s$ Casimir currents. One of the levels for the spin- 1 current is fixed by the positive integer $k$ and the other is fixed by 1 in the numerator of the coset CFT (1.1). One of the specialties of the above coset model (1.1) is that the additional currents generated in the OPEs for the general level $l$ in the second numerator $\mathrm{SU}(N)$ current become null fields for the level $l=1$ and hence decouple [4]. See recent review papers $[5,6]$ for the above duality.

The spin-3 (Casimir) current was reported in [4]. The four independent cubic terms were made from the two spin- 1 currents in the numerator of the coset (1.1). The spin- 4 (Casimir) current was generated [7] by calculating the OPE between this spin-3 Casimir current and itself, which consists of quartic terms and quadratic terms with two derivatives in the above spin- 1 currents. The next step is to determine how one can construct the next higher spin-5 Casimir current. Because the complete form of the spin- 3 and spin- 4 Casimir currents is known, the OPE between the spin- 3 current and spin- 4 current can be calculated using the current algebra in the numerator $\mathrm{SU}(N)$ currents of spin-1. The spin-5 Casimir current can be extracted explicitly from the second-order pole of this OPE. ${ }^{1}$

The explicit form for the spin- 5 current is needed because it was necessary in reference [2] to obtain the commutator between the spin-3 Laurent mode and the spin-4 Laurent mode and determine the minimal representation $[2,5]$ of the asymptotic quantum symmetry algebra $\mathcal{W}_{\infty}[\mu]$ of the higher spin theory on the $A d S_{3}$ space, where $\mu$ is a parameter of the algebra. ${ }^{2}$ The zero mode eigenvalue equations of the spin-5 Casimir current in the

\footnotetext{
${ }^{1}$ This will continue to the generation of general higher spin-s $(s \geq 3)$ currents. In other words, once the structure of the higher spin- $s$ current is known, the OPE between the spin-3 current and this spin- $s$ current will provide the structure of the higher spin- $(s+1)$ current at the second order pole.

${ }^{2}$ The eigenvalues of the zero mode of the spin- 3 and spin- 4 fields were expressed as the conformal spin $h$ of the ground state and the central charge $c$, which is also a parameter of the algebra. Furthermore, the eigenvalue of the zero mode of the spin- 5 field was described as the eigenvalues of the zero mode of the spin-3 and spin- 4 fields, the conformal spin $h$ of the ground state, the central charge $c$ and $\mu$-dependent structure constant appearing in front of the spin-3 field Laurent mode in the right hand side of the above commutator. The cubic equation for the conformal spin $h$ contains two solutions in terms of the two parameters $(\mu, c)$. Under these conditions, $\mu=N$ and $c=c_{N, k}$, where $c_{N, k}$ is equal to (2.8), the above two conformal spins are exactly those of the physical representations, coset primaries denoted by $(f ; 0)$ and $(0 ; f)[1,5]$.
} 
2-dimensional coset model (1.1) behave like those of the spin-5 field in asymptotic quantum symmetry algebra $\mathcal{W}_{\infty}[\mu]$ of the higher spin theory on the $A d S_{3}$ space.

In this paper, the spin-5 Casimir current was obtained and consists of quintic, quartic (with one derivative) and cubic (with two derivatives) WZW currents in the coset (1.1) contracted with $\mathrm{SU}(N)$ invariant tensors. The relative 52 coefficients depend on $N$ and $k$ explicitly. To arrive at this spin-5 current, one should realize that the second order pole of the OPE between the spin- 3 and spin- 4 currents contains the other part as well as the new spin- 5 current. ${ }^{3}$

The structure constant mentioned above can be determined explicitly because this study was interested in the specific WZW model and the basic current algebra is known explicitly. Furthermore, in general, the second order pole after calculating the various OPEs between the spin-3 current with four terms and spin- 4 current with 19 terms contains many unwanted normal ordered products between the fields. They should be rearranged in terms of the fully normal ordered products [34]. This step is necessary because the zero mode for any given multiple product of the fields should be taken very carefully, and the zero mode can be obtained from this fully normal ordered product systematically. The rearrangement lemmas in [34] are useful for obtaining the desired normal ordered product.

The zero modes of the spin- 1 currents that play the role of $\mathrm{SU}(N)$ generators act on the coset primaries. The singlet condition suggests that the zero mode of the spin- 1 current with level $k$ in the numerator of (1.1) acting on the state $|(0 ; f)\rangle$, where the element 0 means a trivial representation of the coset, vanishes while the zero mode of the diagonal spin- 1 current in the denominator of (1.1) acting on the state $|(f ; 0)\rangle$ vanishes [13]. These are the eigenvalue equations, and the rightmost zero mode should be taken first when this singlet condition is applied to the multiple product (quintic, quartic and cubic terms) of zero modes. After that, the $\mathrm{SU}(N)$ generators appear. Then the next rightmost zero mode can be repeated with the singlet condition (each zero mode disappears and the generators with $\mathrm{SU}(N)$ group indices do appear). ${ }^{4}$ At the final stage after calculating this procedure, we are left with the eigenvalue, which has the form of a trace of the $\mathrm{SU}(N)$ generators contracted group indices with a range of $\mathrm{SU}(N)$ invariant tensors, times the state. The eigenvalue depends on $N$.

The relative 52 coefficients appearing in the spin- 5 current are very complicated expressions in terms of $N$ and $k$. This is because they arise from each coefficient function in the spin- 3 current and in the spin- 4 current. The latter consists of rather complicated fractional functions, whereas the former has a simple factorized form. Furthermore, the final

\footnotetext{
${ }^{3}$ How does one subtract this extra piece from the second order pole to obtain the correct spin-5 primary current? Any OPE between the two quasi-primary fields with given spins produce other quasi-primary fields with fixed spins in the right hand side of this OPE [8-12]. Of course, the structure constant is generally unknown. On the other hand, the coefficients arising in the descendant fields of this OPE are determined completely by the spins of these three quasi-primary fields (two of them are the operators in the left hand side and one is the operator in the right hand side) and the number of derivatives in the quasi-primary field in the right hand side.

${ }^{4}$ Note that the state $|(0 ; f)\rangle$ transforms as a fundamental representation with respect to the zero mode of the second numerator current with level 1 and the state $|(f ; 0)\rangle$ transforms as a fundamental representation with respect to the zero mode of the first numerator current with level $k$.
} 
expression for the coefficient functions is rather involved due to ordering in the composite fields. Although each 52 coefficient function is complicated, the eigenvalue equations for the spin- 5 current have very simple factorized forms. ${ }^{5}$

In section 2, after reviewing the GKO coset construction for the spin-2 current, the generalization to the spin-3 and spin-4 currents is described.

In section 3, from the explicit expressions for the spin-3 and spin-4 Casimir currents, the second order pole of the OPE between them is obtained. The new spin-5 Casimir current is found by subtracting both the quasi primary field of spin- 5 and the descendant field (of spin-5) of spin-3 Casimir current with correct coefficient functions.

Section 4 analyzes the zero mode eigenvalue equations of spin- 5 Casimir current in the large $N$ limit. The three-point functions are obtained. The exact eigenvalue equations and the exact three-point functions are also described.

Section 5 presents a summary of this work as well as a discussion on the future directions.

Appendices $A-F$ provide detailed descriptions of sections 3 and 4.

\section{Generalization of the GKO coset construction: review}

The diagonal coset WZW model is given by the following coset $\frac{G}{H}$, which is expressed as

$$
\frac{G}{H}=\frac{\widehat{S U}(N)_{k} \oplus \widehat{S U}(N)_{1}}{\widehat{S U}(N)_{k+1}} .
$$

The numerator spin- 1 currents are denoted by $K^{a}(z)$ of level $k$ and $J^{a}(z)$ of level 1 , whereas the denominator spin- 1 current is denoted by $J^{\prime a}(z)$ of level $(k+1)$. The standard OPE between $J^{a}(z)$ and $J^{b}(w)$ is

$$
J^{a}(z) J^{b}(w)=-\frac{1}{(z-w)^{2}} k_{1} \delta^{a b}+\frac{1}{(z-w)} f^{a b c} J^{c}(w)+\cdots,
$$

where the level is characterized by the positive integer $k_{1}$, which is fixed as 1 in the coset model (2.1). The other spin-1 current with level $k$ living in the other $\mathrm{SU}(N)$ factor of the above coset model (2.1) satisfies the following OPE between $K^{a}(z)$ and $K^{b}(w)$,

$$
K^{a}(z) K^{b}(w)=-\frac{1}{(z-w)^{2}} k_{2} \delta^{a b}+\frac{1}{(z-w)} f^{a b c} K^{c}(w)+\cdots
$$

where the level is given by $k_{2}$, which is rewritten as $k$ in the coset (2.1). These two spin-1 currents are independent of each other because the OPE satisfies $J^{a}(z) K^{b}(w)=0$ and there

\footnotetext{
${ }^{5}$ To reveal the duality between the higher spin theory on the $A d S_{3}$ and $W_{N}$ minimal model coset CFT, the eigenvalues for the higher spin currents in the coset model can be compared with those of the higher spin fields in the asymptotic quantum symmetry algebra $\mathcal{W}_{\infty}[\mu]$ of the higher spin theory on the $A d S_{3}$. As described before, the CFT computations for the eigenvalue equations of the higher spin- 5 current coincide with those in $\mathcal{W}_{\infty}[\mu]$. The corresponding eigenvalue equations for the spin-3 and spin-4 currents (including the spin-2 current) can be calculated at finite $N$ and $k$ (the large $N$ limit result can be obtained by taking the limit appropriately).
} 
are no singular terms between them. The diagonal spin-1 current living in the denominator of the coset (2.1) is the sum of the above two numerator currents

$$
J^{\prime a}(z)=J^{a}(z)+K^{a}(z) .
$$

By adding the OPEs (2.2) and (2.3), the following OPE between the currents (2.4) satisfy

$$
J^{\prime a}(z) J^{\prime b}(w)=-\frac{1}{(z-w)^{2}} k^{\prime} \delta^{a b}+\frac{1}{(z-w)} f^{a b c} J^{\prime c}(w)+\cdots, \quad k^{\prime} \equiv k_{1}+k_{2} \equiv k+1 .
$$

By construction, the level of the denominator current in (2.5) is the sum of each level of the numerator current.

The stress energy tensor with arbitrary levels $\left(k_{1}, k_{2}\right)$ via a Sugawara construction is expressed as

$$
T(z)=-\frac{1}{2\left(k_{1}+N\right)} J^{a} J^{a}(z)-\frac{1}{2\left(k_{2}+N\right)} K^{a} K^{a}(z)+\frac{1}{2\left(k_{1}+k_{2}+N\right)} J^{\prime a} J^{\prime a}(z) .
$$

The corresponding stress energy tensor in the coset model (2.1) can be read off from (2.6). The OPE of the stress energy tensor (2.6) with itself from the OPEs (2.2), (2.3) and (2.5) is given by

$$
T(z) T(w)=\frac{1}{(z-w)^{4}} \frac{c}{2}+\frac{1}{(z-w)^{2}} 2 T(w)+\frac{1}{(z-w)} \partial T(w)+\cdots,
$$

where $c$ in the fourth-order pole of (2.7) denotes the central charge in the coset (2.1) that depends on $N$ and $k$ and is given by

$$
c=(N-1)\left[1-\frac{N(N+1)}{(N+k)(N+k+1)}\right] .
$$

The higher spin-3 current consists of cubic WZW currents contracted with the $\mathrm{SU}(N)$ completely symmetric traceless invariant $d^{a b c}$ tensor. The relative coefficient functions were fixed by two conditions: 1) this spin- 3 current transforms as a primary field under the above stress energy tensor $(2.6)$ with levels $(1, k)$; and 2$)$ the OPE between this spin-3 current and the diagonal current (2.4) does not have any singular terms. Finally, the overall coefficient can be fixed by calculating the highest-order singular term in the spin-3 spin-3 OPE, which is equal to $\frac{c}{3}$ with $(2.8)$.

A higher spin-4 current can be constructed similarly, and consists of quartic, cubic (with one derivative) and quadratic (with two derivative) WZW currents. This spin-4 current can be obtained in two ways. One is to consider the most general spin- 4 current and determine the various unknown coefficient functions using the above two requirements. The other is to calculate the OPE between the above spin-3 current and itself and read off the second-order pole structure. The normalization of the spin- 4 current is related to the normalization of the spin-3 current and the structure constant appearing in the spin- 4 current from the OPE between the spin-3 current and itself. In this case, the structure of the spin- 4 current is determined completely except for the normalization constant because the full expression of the spin-3 current is known. 


\section{Spin-5 Casimir operator}

Subsection 3.1 reviews the relevant facts about the spin-3 and spin- 4 currents first. This subsection will focus on the second-order pole in the OPE between the spin-3 current and the spin- 4 current. Subsection 3.2 then constructs the next higher spin- 5 current from the known spin-3 and spin-4 currents explicitly.

\subsection{Review on the spin-3 and spin- 4 currents}

For later convenience, let us introduce spin-3 operators

$$
Q(z) \equiv d^{a b c} J^{a} J^{b} J^{c}(z), \quad R(z) \equiv d^{a b c} K^{a} K^{b} K^{c}(z),
$$

and spin-2 operators

$$
Q^{a}(z) \equiv d^{a b c} J^{b} J^{c}(z), \quad R^{a}(z) \equiv d^{a b c} K^{b} K^{c}(z) .
$$

As described before, the $d$ symbol appearing in (3.1) and (3.2) is a completely symmetrical traceless $\mathrm{SU}(N)$ invariant tensor of rank 3. The operators $Q(z)$ and $Q^{a}(z)$ are the primary fields of spin-2 and spin-3, respectively, under the stress energy tensor defined in the second numerator $\mathrm{SU}(N)_{1}$ factor in coset (2.1), corresponding to the first term of (2.6). Similarly, the operators, $R(z)$ and $R^{a}(z)$, are the primary fields of spin-2 and spin-3, respectively, under the stress energy tensor defined in the first numerator $\mathrm{SU}(N)_{k}$ factor, corresponding to the second term of (2.6). This can also be generalized to the spin- 4 operators but the $d$ symbol of rank 4 is reduced to the product of the $d$ symbol of rank 3 and the $\delta$ symbol of rank 2 with the appropriate contractions. See equation (A.3).

Calculating the following OPEs is straightforward $[4,34]$

$$
\begin{aligned}
J^{a}(z) Q^{b}(w) & =-\frac{1}{(z-w)^{2}}\left(2 k_{1}+N\right) d^{a b c} J^{c}(w)+\frac{1}{(z-w)} f^{a b c} Q^{c}(w)+\cdots \\
J^{a}(z) Q(w) & =-\frac{1}{(z-w)^{2}} 3\left(k_{1}+N\right) Q^{a}(w)+\cdots \\
K^{a}(z) R^{b}(w) & =-\frac{1}{(z-w)^{2}}\left(2 k_{2}+N\right) d^{a b c} K^{c}(w)+\frac{1}{(z-w)} f^{a b c} R^{c}(w)+\cdots \\
K^{a}(z) R(w) & =-\frac{1}{(z-w)^{2}} 3\left(k_{2}+N\right) R^{a}(w)+\cdots
\end{aligned}
$$

Occasionally, some of the OPEs can be obtained from the known OPEs by taking the symmetries between the currents and levels. For example, the OPE (3.5) can be obtained from the OPE (3.3) using $J^{a} \rightarrow K^{a}$ (and $Q^{a} \rightarrow R^{a}$ ) and $k_{1} \rightarrow k_{2}$ without calculating (3.5) independently and vice versa. Similarly, the OPE (3.6) is related to the OPE (3.4) with $J^{a} \leftrightarrow K^{a}$ and $k_{1} \leftrightarrow k_{2}$. Therefore, some OPEs and some operators are expressed with arbitrary levels $\left(k_{1}, k_{2}\right)$ rather than fixing them.

The coset spin-3 primary field can be expressed as

$$
\begin{aligned}
W^{(3)}(z) & =d^{a b c}\left(A_{1} J^{a} J^{b} J^{c}(z)+A_{2} J^{a} J^{b} K^{c}(z)+A_{3} J^{a} K^{b} K^{c}(z)+A_{4} K^{a} K^{b} K^{c}(z)\right) \\
& \equiv A_{1} Q(z)+A_{2} K^{a} Q^{a}(z)+A_{3} J^{a} R^{a}(z)+A_{4} R(z),
\end{aligned}
$$


where the coefficient functions that depend on $k_{1}, k_{2}$ or $N$ were determined elsewhere [4]

$$
\begin{array}{ll}
A_{1} \equiv k_{2}\left(N+k_{2}\right)\left(N+2 k_{2}\right), & A_{2} \equiv-3\left(N+k_{1}\right)\left(N+k_{2}\right)\left(N+2 k_{2}\right), \\
A_{3} \equiv 3\left(N+k_{1}\right)\left(N+k_{2}\right)\left(N+2 k_{1}\right), & A_{4} \equiv-k_{1}\left(N+k_{1}\right)\left(N+2 k_{1}\right) .
\end{array}
$$

Actually, there is an overall normalization factor $B\left(N, k_{1}, k_{2}\right)$ in $(3.7)$, but $B\left(N, k_{1}, k_{2}\right)$ is not considered. Compared to the stress energy tensor (2.6), the spin-3 current is not the difference between the spin-3 current in the numerator and the spin-3 current in the denominator in the coset model (2.1). As described previously, by assuming the possible terms of spin-3, all the relative coefficient functions are fixed by the above two conditions. The normalization factor $B\left(N, k_{1}, k_{2}\right)$ is determined by calculating the OPE of the spin-3 current and itself. See the equation (4.33) for the explicit form.

By substituting (A.2) and (A.4) into (A.1), the following form of the spin-4 current was obtained

$$
\begin{aligned}
W^{(4)}(z)= & d^{a b e} d^{c d e}\left(h_{6} J^{a} J^{b} J^{c} J^{d}+h_{7} J^{a} J^{b} J^{c} K^{d}+h_{8} J^{a} J^{b} K^{c} K^{d}+h_{9} J^{a} K^{b} K^{c} K^{d}\right. \\
& \left.+h_{10} K^{a} K^{b} K^{c} K^{d}\right)(z)+h_{11} J^{a} J^{a} J^{b} J^{b}(z)+h_{12} J^{a} J^{a} J^{b} K^{b}(z)+h_{13} J^{a} J^{a} K^{b} K^{b}(z) \\
& +h_{14} J^{a} K^{a} K^{b} K^{b}(z)+h_{15} K^{a} K^{a} K^{b} K^{b}(z)+h_{17} \partial^{2} J^{a} K^{a}(z)+h_{20} \partial J^{a} \partial K^{a}(z)+h_{22} J^{a} \partial^{2} K^{a}(z) \\
& +h_{23} J^{a} J^{b} K^{a} K^{b}(z)+h_{24} d^{a c e} d^{b d e} J^{a} J^{b} K^{c} K^{d}(z)+h_{25} f^{a b c} \partial J^{a} J^{b} K^{c}(z)+h_{26} f^{a b c} \partial K^{a} K^{b} J^{c}(z) \\
& +h_{27} \partial^{2} J^{a} J^{a}(z)+h_{28} \partial^{2} K^{a} K^{a}(z)+h_{29} \partial J^{a} \partial J^{a}(z)+h_{30} \partial K^{a} \partial K^{a}(z),
\end{aligned}
$$

where the 21 coefficient functions are

$$
\begin{aligned}
& h_{6} \equiv c_{6}+3 c_{1}, \quad h_{7} \equiv c_{7}+3 c_{2}, \quad h_{8} \equiv c_{8}+c_{3}, \quad h_{9} \equiv c_{9}+3 c_{4}, \quad h_{10} \equiv c_{10}+3 c_{5}, \\
& h_{11} \equiv c_{11}-\frac{12\left(N^{2}-4\right)}{N\left(N^{2}+1\right)} c_{1}, \quad h_{12} \equiv c_{12}-\frac{12\left(N^{2}-4\right)}{N\left(N^{2}+1\right)} c_{2}, \quad h_{13} \equiv c_{13}-\frac{4\left(N^{2}-4\right)}{N\left(N^{2}+1\right)} c_{3}, \\
& h_{14} \equiv c_{14}-\frac{12\left(N^{2}-4\right)}{N\left(N^{2}+1\right)} c_{4}, \quad h_{15} \equiv c_{15}-\frac{12\left(N^{2}-4\right)}{N\left(N^{2}+1\right)} c_{5}, \\
& h_{17} \equiv c_{17}+\frac{2\left(N^{2}-4\right)\left(N^{2}-3\right)}{N^{2}+1} c_{2}, \quad h_{20} \equiv c_{20}-\frac{\left(N^{2}-4\right)\left(N^{2}-3\right)}{N^{2}+1} c_{3}, \\
& h_{22} \equiv c_{22}-\frac{\left(N^{2}-4\right)\left(N^{2}-3\right)}{N^{2}+1} c_{4}, \quad h_{23} \equiv c_{23}-\frac{8\left(N^{2}-4\right)}{N\left(N^{2}+1\right)} c_{3}, \quad h_{24} \equiv 2 c_{3}, \\
& h_{25} \equiv-\frac{3\left(N^{2}-4\right)\left(N^{2}-3\right)}{N\left(N^{2}+1\right)} c_{2}, \quad h_{26} \equiv \frac{3\left(N^{2}-4\right)\left(N^{2}-3\right)}{N\left(N^{2}+1\right)} c_{4}, \\
& h_{27} \equiv \frac{2\left(N^{2}-4\right)\left(N^{2}-3\right)}{N^{2}+1} c_{1}, \quad h_{28} \equiv \frac{2\left(N^{2}-4\right)\left(N^{2}-3\right)}{N^{2}+1} c_{5}, \\
& h_{29} \equiv-\frac{3\left(N^{2}-4\right)\left(N^{2}-3\right)}{N^{2}+1} c_{1}, \quad h_{30} \equiv-\frac{3\left(N^{2}-4\right)\left(N^{2}-3\right)}{N^{2}+1} c_{5} .
\end{aligned}
$$

The last seven-terms of (3.9) newly appear and the first five-terms of (A.1) disappear. From (3.10), the precise relationships between the spin-4 current (A.1) and its different versions (3.9) and the subscripts of the $h$ coefficients are kept unchanged.

\subsection{The spin-5 current}

The next step is to construct the spin-5 current from the spin-3 current (3.7) and spin-4 current (3.9). Although in principle the complete OPE can be calculated, this subsection focuses on the second order pole only to extract the spin- 5 current. The OPE between the 
spin-3 and spin-4 currents is given by [2]

$$
\begin{aligned}
W^{(3)}(z) W^{(4)}(w)= & \frac{1}{(z-w)^{4}} \eta_{W} W^{(3)}(w)+\frac{1}{(z-w)^{3}} \frac{1}{3} \eta_{W} \partial W^{(3)}(w) \\
& +\frac{1}{(z-w)^{2}}\left[\eta_{\Lambda}\left(T W^{(3)}-\frac{3}{14} \partial^{2} W^{(3)}\right)+\frac{\eta_{W}}{14} \partial^{2} W^{(3)}+W^{(5)}\right](w) \\
& +\left(\mathcal{O}(z-w)^{-1}\right)
\end{aligned}
$$

where the $(N, c)$-dependent coefficients are

$$
\eta_{\Lambda}=\frac{39}{(114+7 c)}\left(C_{33}^{4}\right)^{2}, \quad \eta_{W}=\frac{3}{4}\left(C_{33}^{4}\right)^{2} .
$$

Because the primary currents are not normalized properly, there is little difference from [2] in an overall factor

$$
\left.W^{(3)}(z) W^{(4)}(w)\right|_{\frac{1}{(z-w)^{2}}}=\eta_{\Lambda}\left(T W^{(3)}(w)-\frac{3}{14} \partial^{2} W^{(3)}(w)\right)+\frac{\eta_{W}}{14} \partial^{2} W^{(3)}(w)+W^{(5)}(w) .
$$

The combination $\left(T W^{(3)}-\frac{3}{14} \partial^{2} W^{(3)}\right)(w) \equiv \Lambda(w)$ in (3.12) is a quasi-primary field. ${ }^{6}$ The explicit $(N, c)$-dependence of the structure constant $C_{33}^{4}$ in (3.11) was obtained from the literature $[2,16]$

$$
\left(C_{33}^{4}\right)^{2}=\frac{64(c+2)(N-3)[c(N+3)+2(4 N+3)(N-1)]}{(5 c+22)(N-2)[c(N+2)+(3 N+2)(N-1)]} .
$$

Note that due to the $(N-3)$ factor in (3.13), the coset model (2.1) for $N=3$ does not produce the spin- 4 current. The spin- 5 current appearing in the OPE between the spin- 3 and the spin- 4 currents should contain the factor $(N-4)$ in its expression. The $N$ should be greater than 4 . This issue will be addressed later. See equation (4.33).

The spin-5 current can be obtained from

$$
W^{(5)}(w)=\left.W^{(3)}(z) W^{(4)}(w)\right|_{\frac{1}{(z-w)^{2}}}-\eta_{\Lambda}\left(T W^{(3)}(w)-\frac{3}{14} \partial^{2} W^{(3)}(w)\right)-\frac{\eta_{W}}{14} \partial^{2} W^{(3)}(w) .
$$

The stress energy tensor and the spin-3 current are given in (2.6) and (3.7). Furthermore, the relative coefficients are given in (3.11) together with (3.13). To ensure the right relative coefficients in the right hand side of (3.14), two arbitrary unknown constants can be introduced in front of $\eta_{\Lambda}$ and $\eta_{W}$. For $N=3$, they are vanishing by calculating the OPE between $T(z)$ and $W^{(5)}(w)$ and requiring $W^{(5)}(w)$ is a primary field of spin-5. This is consistent with the fact that the structure constant $C_{33}^{4}$ in (3.13) vanishes at $N=3$. For $N=4$ and $N=5$, these extra parameters are equal to 1 by solving the primary condition.

\footnotetext{
${ }^{6}$ The coefficient $\frac{3}{14}$ comes from general formula $\frac{3}{2(2 s+1)}$, where $s$ is the spin for the field $\Phi[9,12]$. In other words, the OPE $T(z) \Lambda(w)$ has no third order pole and the nonvanishing fourth order pole of this OPE is given by $\frac{1}{14}(114+7 c) W^{(3)}$. The numerical factor $\frac{1}{14}$ can be obtained from the formula $\frac{1}{n !} \prod_{x=0}^{n-1} \frac{\left(h_{i}-h_{j}+h_{k}+x\right)}{\left(2 h_{k}+x\right)}$ with $h_{i}=3, h_{j}=4, h_{k}=3$ and $n=2[9,12]$. Note that the equation, $C_{34}^{3}=\frac{3}{4} C_{33}^{4}$ holds for general $N$. Furthermore, for $N=4$, expression (3.12) without the spin-5 current is already known in [9, 14, 15].
} 
The next step is to calculate the second order pole for the $\operatorname{OPE} W^{(3)}(z) W^{(4)}(w)$ explicitly. Appendix $B$ presents all the detailed calculations. Appendix $C$ describes the fully normal ordering procedure. For $N=3$ or $N=4$, all the calculations in appendices $B$ and $C$ were checked explicitly using Thielemans package [17].

After the complicated calculations, the spin- 5 current is summarized by the following 52 terms:

$$
\begin{aligned}
& W^{(5)}(z)=a_{1} d^{a b f} d^{f c g} d^{g d e} J^{a} J^{b} J^{c} J^{d} J^{e}(z)+\left(a_{2} d^{a b f} d^{f c g} d^{g d e}+a_{3} d^{a b f} d^{f e g} d^{g c d}\right) J^{a} J^{b} J^{c} J^{d} K^{e}(z) \\
& +\left(a_{4} d^{a b f} d^{f c g} d^{g d e}+a_{5} d^{b d f} d^{f a g} d^{g c e}+a_{6} d^{a b f} d^{f d g} d^{g c e}\right) J^{a} J^{b} J^{c} K^{d} K^{e}(z) \\
& +\left(a_{7} d^{a b f} d^{f c g} d^{g d e}+a_{8} d^{b c f} d^{f a g} d^{g d e}+a_{9} d^{a c f} d^{f d g} d^{g b e}\right) J^{a} J^{b} K^{c} K^{d} K^{e}(z) \\
& +\left(a_{10} d^{a b f} d^{f c g} d^{g d e}+a_{11} d^{b c f} d^{f a g} d^{g d e}\right) J^{a} K^{b} K^{c} K^{d} K^{e}(z)+a_{12} d^{a b f} d^{f c g} d^{g d e} K^{a} K^{b} K^{c} K^{d} K^{e}(z) \\
& +a_{13} d^{a b c} \delta^{d e} J^{a} J^{b} J^{c} J^{d} J^{e}(z)+\left(a_{14} d^{a b c} \delta^{d e}+a_{15} d^{a b e} \delta^{c d}\right) J^{a} J^{b} J^{c} J^{d} K^{e}(z) \\
& +\left(a_{16} d^{a b c} \delta^{d e}+a_{17} d^{a b d} \delta^{c e}+a_{18} \delta^{a b} d^{c d e}\right) J^{a} J^{b} J^{c} K^{d} K^{e}(z) \\
& +\left(a_{19} d^{a b c} \delta^{d e}+a_{20} \delta^{a b} d^{c d e}+a_{21} \delta^{a c} d^{b d e}\right) J^{a} J^{b} K^{c} K^{d} K^{e}(z) \\
& +\left(a_{22} d^{a b c} \delta^{d e}+a_{23} \delta^{a b} d^{c d e}\right) J^{a} K^{b} K^{c} K^{d} K^{e}(z)+a_{24} d^{a b c} \delta^{d e} K^{a} K^{b} K^{c} K^{d} K^{e}(z) \\
& +a_{25} f^{a d e} d^{b c e} J^{a} J^{b} J^{c} \partial J^{d}(z)+a_{26} d^{a b e} f^{c d e} J^{a} J^{b} J^{c} \partial K^{d}(z)+\left(a_{27} d^{a b e} f^{c d e}+a_{28} d^{a c e} f^{b d e}\right. \\
& \left.+a_{29} d^{a d e} f^{b c e}\right) J^{a} J^{b} \partial J^{c} K^{d}(z)+\left(a_{30} d^{a b e} f^{c d e}+a_{31} d^{a c e} f^{b d e}+a_{32} d^{a d e} f^{b c e}\right) J^{a} J^{b} K^{c} \partial K^{d}(z) \\
& +\left(a_{33} d^{a c e} f^{b d e}+a_{34} f^{a d e} d^{b c e}+a_{35} f^{a b e} d^{c d e}\right) J^{a} \partial J^{b} K^{c} K^{d}(z)+\left(a_{36} d^{a b e} f^{c d e}+a_{37} f^{a d e} d^{b c e}\right. \\
& \left.+a_{38} f^{a c e} d^{b d e}\right) J^{a} K^{b} K^{c} \partial K^{d}(z)+a_{39} f^{a d e} d^{b c e} \partial J^{a} K^{b} K^{c} K^{d}(z)+a_{40} f^{a d e} d^{b c e} K^{a} K^{b} K^{c} \partial K^{d}(z) \\
& +a_{41} d^{a b c} J^{a} J^{b} \partial^{2} J^{c}(z)+a_{42} d^{a b c} J^{a} \partial J^{b} \partial J^{c}(z)+a_{43} d^{a b c} J^{a} \partial^{2} J^{b} K^{c}(z)+a_{44} d^{a b c} J^{a} J^{b} \partial^{2} K^{c}(z) \\
& +a_{45} d^{a b c} \partial J^{a} \partial J^{b} K^{c}(z)+a_{46} d^{a b c} J^{a} \partial J^{b} \partial K^{c}(z)+a_{47} d^{a b c} \partial^{2} J^{a} K^{b} K^{c}(z) \\
& +a_{48} d^{a b c} J^{a} K^{b} \partial^{2} K^{c}(z)+a_{49} d^{a b c} \partial J^{a} K^{b} \partial K^{c}(z)+a_{50} d^{a b c} J^{a} \partial K^{b} \partial K^{c}(z) \\
& +a_{51} d^{a b c} K^{a} K^{b} \partial^{2} K^{c}(z)+a_{52} d^{a b c} K^{a} \partial K^{b} \partial K^{c}(z),
\end{aligned}
$$

where the 52 coefficient functions $a_{i}$ are given in appendix $D$ (D.1). The spin- 5 current (3.15) at $N=5$ was checked to confirm that there are no singular terms (in the OPE between the stress energy tensor and the spin-5 current) with an order greater than 2. For consistency check, the OPE between the spin-5 current and the diagonal current was also calculated, which showed that are no singular terms when $N=4$. The primary condition with $N=4$ was checked until the third order singular terms. Furthermore, when $N=3$, the above regularity and primary conditions (up to the second order pole) were checked explicitly. The spin- 5 current consists of the first 24 quintic terms, the middle sixteen quartic terms with the $f$ symbol (with one derivative), and the remaining twelve cubic terms with the two derivatives. The last twelve-terms can be observed from the second derivative of spin-3 current $W^{(3)}(z)$. The twelve quintic terms containing the $\delta$ symbol also can be seen from the composite operator, $T W^{(3)}(z)$. The vacuum character (7.18) of [3], which is equal to $\frac{1}{\prod_{s=2}^{N} \prod_{n=s}^{\infty}\left(1-q^{n}\right)}=1+q^{2}+2 q^{3}+4 q^{4}+6 q^{5}+12 q^{6}+\mathcal{O}\left(q^{7}\right)$, contains the numerical factor, 6 , in front of the $q^{5}$ term. This suggests that there are six spin-5 fields, $T W^{(3)}(z), \partial^{2} W^{(3)}(z)$, $\partial W^{(4)}(z), \partial^{3} T(z), \partial T^{2}(z)$, and $W^{(5)}(z)$. Among these fields, the only primary spin-5 field is given by $W^{(5)}(z)$. For the next higher spin-6 fields, there are eleven nonprimary fields, 
which can be obtained from the higher spin currents of spin $s=3,4,5$, the stress energy tensor, and derivatives, and a single spin-6 primary current.

\section{Three-point functions}

\subsection{Eigenvalue equation of the zero mode of spin-5 current acting on the state $|(f ; 0)\rangle$ in the large $N$ limit}

This section describes the three-point functions with scalars for the spin-5 current found in the previous section. The large $N$ 't Hooft limit [1] is defined as

$$
N, k \rightarrow \infty, \quad \lambda \equiv \frac{N}{N+k} \quad \text { fixed }
$$

The product of the $\mathrm{SU}(N)$ generators has the following decompositions with $\delta, d$ and $f$ symbols (for example, see reference [7])

$$
T^{a} T^{b}=-\frac{1}{N} \delta^{a b}-\frac{i}{2} d^{a b c} T^{c}+\frac{1}{2} f^{a b c} T^{c} .
$$

A range of quintic products with three $d$ symbols can be obtained from equation (4.2) and the traceless condition for the anti-hermitian basis.

The zero modes of the current satisfy the commutation relations of the underlying finite dimensional Lie algebra $\mathrm{SU}(N)$. For the state $|(f ; 0)\rangle, T^{a}$ corresponds to $K_{0}^{a}$ and for the state $|(0 ; f)\rangle, T^{a}$ corresponds to $J_{0}^{a}$ as follows

$$
|(f ; 0)\rangle: \quad T^{a} \leftrightarrow K_{0}^{a}, \quad|(0 ; f)\rangle: \quad T^{a} \leftrightarrow J_{0}^{a} .
$$

\subsubsection{Eigenvalue equation}

Now the zero mode eigenvalue equation of the spin 5 primary field $W^{(5)}(z)$ acting on the primary state $(f ; 0)$ in the large $N$ 't Hooft limit can be calculated using the relations in appendix (D.3). The ground state transforms as a fundamental representation with respect to $K_{0}^{a}$ (and as an antifundamental representation with respect to $J_{0}^{a}$ ). For example, calculate the following eigenvalue equation corresponding to the first term of the spin- 5 current

$$
d^{a b f} d^{f c g} d^{g d e}\left(J^{a} J^{b} J^{c} J^{d} J^{e}\right)_{0}|(f ; 0)\rangle .
$$

How does one obtain the zero mode of the composite operator? Using the standard definition for the zero mode, (A.2) of [34],

$$
d^{a b f} d^{f c g} d^{g d e} J_{0}^{e} J_{0}^{d} J_{0}^{c} J_{0}^{b} J_{0}^{a}|(f ; 0)\rangle .
$$

That is, the zero mode is the product of each zero mode but the ordering is reversed (the indices of $a, b, c, d$, and $e$ go to the indices of $e, d, c, b$ and $a$ ). The next step is to use the eigenvalue equation. Because the singlet condition for the state $(f ; 0)$ is expressed as

$$
\left(J_{0}^{a}+K_{0}^{a}\right)|(f ; 0)\rangle=0
$$


The expression (4.4) is equivalent to

$$
-d^{a b f} d^{f c g} d^{g d e} J_{0}^{e} J_{0}^{d} J_{0}^{c} J_{0}^{b} K_{0}^{a}|(f ; 0)\rangle=-d^{a b f} d^{f c g} d^{g d e} K_{0}^{a} J_{0}^{e} J_{0}^{d} J_{0}^{c} J_{0}^{b}|(f ; 0)\rangle
$$

where the zero mode $K_{0}^{a}$ commutes with the zero mode $J_{0}^{b}$ and $K_{0}^{a}$ is moved to the left. The singlet condition (4.5) is applied to the rightmost zero mode $J_{0}^{b}(4.6)$ and the following result can be obtained

$$
d^{a b f} d^{f c g} d^{g d e} K_{0}^{a} J_{0}^{e} J_{0}^{d} J_{0}^{c} K_{0}^{b}|(f ; 0)\rangle=d^{a b f} d^{f c g} d^{g d e} K_{0}^{a} K_{0}^{b} J_{0}^{e} J_{0}^{d} J_{0}^{c}|(f ; 0)\rangle .
$$

This procedure can be repeated until all the $J_{0}^{a}$ 's are exchanged with the $K_{0}^{a}$ 's with the appropriate ordering. Therefore, the above eigenvalue equation (4.3) becomes

$$
\begin{aligned}
-d^{a b f} d^{f c g} d^{g d e} K_{0}^{a} K_{0}^{b} K_{0}^{c} K_{0}^{d} K_{0}^{e}|(f ; 0)\rangle & =-\frac{1}{N} d^{a b f} d^{f c g} d^{g d e} \operatorname{Tr}\left(T^{a} T^{b} T^{c} T^{d} T^{e}\right)|(f ; 0)\rangle \\
& \rightarrow i N^{4}|(f ; 0)\rangle .
\end{aligned}
$$

In the first line of (4.8), $\frac{1}{N}$ is multiplied because the eigenvalue is needed (not the trace). Of course, the zero mode eigenvalue equation can be expressed using the zero mode $J_{0}^{a}$ rather than $K_{0}^{a}$ with the corresponding $\mathrm{SU}(N)$ generator rather than $T^{a} \cdot{ }^{7}$ Here the following quantity (related to the first term of spin-5 current) can be used:

$$
\begin{aligned}
d^{a b f} d^{f c g} d^{g d e} \operatorname{Tr}\left(T^{a} T^{b} T^{c} T^{d} T^{e}\right) & =-\frac{i}{8} d^{a b f} d^{f c g} d^{g d e} d^{a b h} d^{h c i} d^{i d e} \\
& =-\frac{i}{8} \frac{2}{N}\left(N^{2}-4\right) \delta^{f h} \frac{2}{N}\left(N^{2}-4\right) \delta^{g i} d^{f c g} d^{h c i} \\
& =-i \frac{1}{N^{3}}\left(N^{2}-4\right)^{3}\left(N^{2}-1\right) \rightarrow-i N^{5},
\end{aligned}
$$

where the identities of appendix $A$ of [7], (A.3) and (A.4), are used. Note $\delta^{a a}=N^{2}-1$. For $N=3$, the above identity is checked explicitly. At the final stage, a large $N$ limit (4.1) is taken. Similarly, the second-, third-, fourth-, sixth-, seventh-, ninth-, tenth-, eleventhand twelfth-terms of the spin- 5 current can be analyzed.

One can proceed to calculate the remaining trace identities. For the fifth-term (and eighth-term) of the spin-5 current, the following calculation can be performed:

$$
\begin{aligned}
d^{a c f} d^{f b g} d^{g d e} \operatorname{Tr}\left(T^{a} T^{b} T^{c} T^{d} T^{e}\right) & =d^{a c f} d^{f b g} d^{g d e}\left(-\frac{i}{2 N} \delta^{a b} d^{c d e}-\frac{i}{8} d^{a b h} d^{h c i} d^{i d e}+\frac{i}{8} f^{a b h} f^{h c i} d^{i d e}\right) \\
& =\frac{4}{N^{3}} i\left(N^{2}-4\right)^{2}\left(N^{2}-1\right) \rightarrow 4 i N^{3} .
\end{aligned}
$$

${ }^{7}$ Similarly, the following eigenvalue equation corresponding to the ninth-term of the spin-5 current can be obtained

$$
\begin{aligned}
d^{a c f} d^{f d g} d^{g b e}\left(J^{a} J^{b} K^{c} K^{d} K^{e}\right)_{0}|(f ; 0)\rangle & =\frac{1}{N} d^{a b f} d^{f c g} d^{g d e} \operatorname{Tr}\left(T^{a} T^{b} T^{c} T^{d} T^{e}\right)|(f ; 0)\rangle \\
& \rightarrow-i N^{4}|(f ; 0)\rangle .
\end{aligned}
$$

In the first line of (4.9), the zero mode is obtained by changing the ordering of the current reversely. The singlet condition (4.5) is used in the second line. There is no change in sign because of the even number of $J_{0}^{a}$ 's. In the third line, $\frac{1}{N}$ is multiplied and the cyclic property of the trace is used. Finally, the previous result of (4.10) is used in the last line of (4.9). 
The $d d d$ product can be reduced to a single $d$ and the $d d f$ product can be written in terms of a single $f$ using the identities involving the $f$-and $d$-tensors of $\mathrm{SU}(N)$. For the $N=3$, the above identity has been checked explicitly. The large $N$ behavior of (4.11) is different from that of (4.10). The large $N$ limit for the coefficient functions was not considered. Once the $N$ behavior of these coefficient functions is included, (4.10) and (4.11) do to the final eigenvalue equations.

For the thirteenth-term (fourteenth-, $\cdots, 24$ th-terms) of the spin-5 current, the quintic product with $d$ symbol and $\delta$ symbol is obtained as follows:

$$
\begin{aligned}
d^{a b c} \delta^{d e} \operatorname{Tr}\left(T^{a} T^{b} T^{c} T^{d} T^{e}\right) & =d^{a b c} \delta^{d e}\left(-\frac{i}{2 N} d^{a b c} \delta^{d e}\right)=-\frac{i}{N^{2}}\left(N^{2}-4\right)\left(N^{2}-1\right)^{2} \\
& \rightarrow-i N^{4}
\end{aligned}
$$

Note that the $d d$ product becomes a single $\delta$ symbol. The large $N$ behavior of (4.12) is different from (4.10) or (4.11). For $N=3$, the above identity has been checked explicitly.

One has the quartic products with $d$ symbol and $f$ symbol for the 25th-term (26th-, 27th-, 29th-, 30th-, 31st-, 33rd-, 35th-, 36th-, 37th-, 39th-, 40th-terms) of the spin-5 current

$$
\begin{aligned}
d^{a b e} f^{c d e} \operatorname{Tr}\left(T^{a} T^{b} T^{c} T^{d}\right) & =\frac{i}{4} d^{a b e} f^{c d e} d^{a b f} f^{c d f}=i\left(N^{2}-4\right)\left(N^{2}-1\right) \\
& \rightarrow i N^{4}
\end{aligned}
$$

The large $N$ behavior of (4.13) is the same as that of (4.12).

For the 28th-term of the spin-5 current,

$$
\begin{aligned}
d^{a c e} f^{b d e} \operatorname{Tr}\left(T^{a} T^{b} T^{c} T^{d}\right) & =d^{a c e} f^{b d e}\left(\frac{i}{4} d^{a b f} f^{c d f}+\frac{i}{4} f^{a b f} d^{c d f}\right) \\
& =\frac{i}{4} d^{a c e}\left(f^{b d e} d^{a b f} f^{c d f}+f^{b d e} f^{a b f} d^{c d f}\right) \\
& =\frac{i}{4} d^{a c e}\left(-N d^{a c e}+N d^{a c e}\right)=0 .
\end{aligned}
$$

The $d f f$ product reduces to a single $d$ symbol. Furthermore, the contributions from the 32nd-, 34th-, and 38th-terms vanish. For $N=3,4$, the above identities (4.13) and (4.14) have been checked explicitly.

Finally, the cubic product with the $d$ symbol is calculated for the 41st-term of the spin-5 current (and 42nd-term, $\cdots$, 52nd-term),

$$
d^{a b c} \operatorname{Tr}\left(T^{a} T^{b} T^{c}\right)=\frac{i}{2} d^{a b c} d^{a b c}=\frac{i}{N}\left(N^{2}-4\right)\left(N^{2}-1\right) \rightarrow i N^{3} .
$$

Moreover, the large $N$ behaviors of spin- 5 coefficient functions $a_{i}$ in the large $N$ limit are given in appendix (D.3).

Let us move on the zero mode eigenvalue with one derivative corresponding to the 25 th-term of the spin-5 current

$$
\begin{aligned}
f^{\text {ade }} d^{\text {bce }}\left(J^{a} J^{b} J^{c} \partial J^{d}\right)_{0}|(f ; 0)\rangle & =f^{a d e} d^{b c e}\left(\partial J^{d}\right)_{0} J_{0}^{c} J_{0}^{b} J_{0}^{a}|(f ; 0)\rangle=-f^{a d e} d^{b c e} J_{0}^{d} J_{0}^{c} J_{0}^{b} J_{0}^{a}|(f ; 0)\rangle \\
& =-f^{a d e} d^{b c e} K_{0}^{a} K_{0}^{b} K_{0}^{c} K_{0}^{d}|(f ; 0)\rangle
\end{aligned}
$$




$$
=\frac{1}{N} d^{a b e} f^{c d e} \operatorname{Tr}\left(T^{a} T^{b} T^{c} T^{d}\right)|(f ; 0)\rangle \rightarrow i N^{3}|(f ; 0)\rangle .
$$

In the first line of (4.16), the zero mode is taken by reversing the ordering of the current as before. One then uses the following property, $\partial J^{a}(z)=\partial\left(\sum_{m} \frac{J_{m}^{a}}{z^{m+1}}\right)=\sum_{m} \frac{(-m-1) J_{m}^{a}}{z^{m+2}} \equiv$ $\sum_{m} \frac{\left(\partial J^{a}\right)_{m}}{z^{m+2}}$, which leads to the zero mode, $\left(\partial J^{a}\right)_{0}=-J_{0}^{a}$. In the second line, the singlet condition (4.5) is used, and there is no sign change due to the even number of the $J_{0}^{a}$ 's. Furthermore, the previous result (4.13) is used. ${ }^{8}$

In this way, the nonzero zero mode contributions of all the terms in $W^{(5)}(z)(3.15)$ can be obtained.

Let us describe the nonzero contributions. From the analysis in (E.1)(E.4), (E.6), (E.7), (E.9)-(E.11), these nine-terms behave as $N^{4}$ and each coefficient function in (D.4) has a factor, $N$. These terms then contribute to the final result in the large $N$ limit. Furthermore, the non-zero contributions from (E.25)-(E.27), (E.29)(E.31), (E.33), (E.35)-(E.37) and (E.39) have a factor, $N^{3}$, and the coefficient functions for these terms behave as $N^{2}$ from (D.4). Finally, from (E.41)-(E.50), these contributions are given by $N^{2}$ and the corresponding coefficients behave as $N^{3}$ from (D.4). By summing these contributions, the following eigenvalue equation can be obtained for the zero mode of the spin-5 current $W_{0}^{(5)}$ acting on the state $|(f ; 0)\rangle$ in the large $N$ limit

$$
W_{0}^{(5)}|(f ; 0)\rangle=-\left[\frac{24 i(1+\lambda)(3+\lambda)(4+\lambda)}{7 \lambda^{3}(2-\lambda)}\right] N^{5}|(f ; 0)\rangle .
$$

The eigenvalue has a very simple factorized form. The same eigenvalue equation at finite $N$ and $k$ will be evaluated.

\subsection{Eigenvalue equation of the zero mode of the spin-5 current acting on the state $|(0 ; f)\rangle$ in the large $N$ limit}

Next the zero mode eigenvalue of the spin-5 primary field $W^{(5)}(z)$ acting on the primary state $|(0 ; f)\rangle$ is described in a large $N$ 't Hooft limit using the above (4.10)-(4.15) relations and appendix (D.3). The ground state transforms as a fundamental representation with respect to $J_{0}^{a}$ and the zero mode $K_{0}^{a}$ has a vanishing eigenvalue equation (i.e. a fundamental representation with respect to the zero mode of the diagonal current)

$$
K_{0}^{a}|(0 ; f)\rangle=0 .
$$

${ }^{8}$ Consider the contribution from the 41 st-term of spin-5 current with two derivative terms

$$
\begin{aligned}
d^{a b c}\left(J^{a} J^{b} \partial^{2} J^{c}\right)_{0}|(f ; 0)\rangle & =d^{a b c}\left(\partial^{2} J^{c}\right)_{0} J_{0}^{b} J_{0}^{a}|(f ; 0)\rangle=2 d^{a b c} J_{0}^{c} J_{0}^{b} J_{0}^{a}|(f ; 0)\rangle \\
& =-2 d^{a b c} K_{0}^{a} K_{0}^{b} K_{0}^{c}|(f ; 0)\rangle=-\frac{2}{N} d^{a b c} \operatorname{Tr}\left(T^{a} T^{b} T^{c}\right)|(f ; 0)\rangle \\
& \rightarrow-2 i N^{2}|(f ; 0)\rangle .
\end{aligned}
$$

In the first line of (4.17), the zero mode is taken. The zero mode of the second derivative for the current can be obtained from the fact that $\partial^{2} J^{a}(z)=\partial^{2}\left(\sum_{m} \frac{J_{m}^{a}}{z^{m+1}}\right)=\sum_{m} \frac{(m+1)(m+2) J_{m}^{a}}{z^{m+3}} \equiv \sum_{m} \frac{\left(\partial^{2} J^{a}\right)_{m}}{z^{m+3}}$, which leads to $\left(\partial^{2} J^{a}\right)_{0}=2 J_{0}^{a}$. In the second line, the singlet condition (4.5) is used. Note the extra minus sign because of the odd number of the current. As before, $\frac{1}{N}$ is multiplied. At the final stage, the result (4.15) is used. Appendix $E$ describes the complete results for the eigenvalue equations in the spin- 5 zero modes. 
For example, calculate the following eigenvalue equation corresponding to the first-term of the spin-5 current

$$
\begin{aligned}
d^{a b f} d^{f c g} d^{g d e}\left(J^{a} J^{b} J^{c} J^{d} J^{e}\right)_{0}|(0 ; f)\rangle & =d^{a b f} d^{f c g} d^{g d e} J_{0}^{e} J_{0}^{d} J_{0}^{c} J_{0}^{b} J_{0}^{a}|(0 ; f)\rangle \\
& =\frac{1}{N} d^{a b f} d^{f c g} d^{g d e} \operatorname{Tr}\left(T^{e} T^{d} T^{c} T^{b} T^{a}\right)|(0 ; f)\rangle,
\end{aligned}
$$

where the previous result (4.4) is used. Equation (4.20) becomes the following expression using the property of the $d$ symbol

$$
\begin{aligned}
d^{a b f} d^{f c g} d^{g d e}\left(J^{a} J^{b} J^{c} J^{d} J^{e}\right)_{0}|(0 ; f)\rangle & =\frac{1}{N} d^{a b f} d^{f c g} d^{g d e} \operatorname{Tr}\left(T^{a} T^{b} T^{c} T^{d} T^{e}\right)|(0 ; f)\rangle \\
& \rightarrow-i N^{4}|(0 ; f)\rangle .
\end{aligned}
$$

The next non-zero contribution appears in the thirteenth-term. The following nontrivial contribution can be obtained from the thirteenth-term

$$
\begin{aligned}
d^{a b c} \delta^{d e}\left(J^{a} J^{b} J^{c} J^{d} J^{e}\right)_{0}|(0 ; f)\rangle & =d^{a b c} \delta^{d e} J_{0}^{e} J_{0}^{d} J_{0}^{c} J_{0}^{b} J_{0}^{a}|(0 ; f)\rangle \\
& =\frac{1}{N} d^{a b c} \delta^{d e} \operatorname{Tr}\left(T^{a} T^{b} T^{c} T^{d} T^{e}\right)|(0 ; f)\rangle \\
& \rightarrow-i N^{3}|(0 ; f)\rangle,
\end{aligned}
$$

where, as before, the trace property is used and the $\frac{1}{N}$ is multiplied to obtain the eigenvalue. The large $N$ limit is taken at the final stage in (4.22). On the other hand, due to the $N$ factor from (D.4), the final contribution becomes zero in the large $N$ limit because $N \times N^{3}=N^{4}$ (the leading large $N$ behavior has $N^{5}$ ).

The next nonzero contribution appears in the 25th-term as follows:

$$
\begin{aligned}
f^{a d e} d^{b c e}\left(J^{a} J^{b} J^{c} \partial J^{d}\right)_{0}|(0 ; f)\rangle & =-f^{a d e} d^{b c e} J_{0}^{d} J_{0}^{c} J_{0}^{b} J_{0}^{a}|(0 ; f)\rangle \\
& =-\frac{1}{N} d^{a b e} f^{c d e} \operatorname{Tr}\left(T^{a} T^{b} T^{c} T^{d}\right)|(0 ; f)\rangle \\
& \rightarrow-i N^{3}|(0 ; f)\rangle .
\end{aligned}
$$

In the first line, the previous relation (4.16) is used. The nonzero contribution in the 41st-term can be obtained

$$
\begin{aligned}
d^{a b c}\left(J^{a} J^{b} \partial^{2} J^{c}\right)_{0}|(0 ; f)\rangle & =2 d^{a b c} J_{0}^{a} J_{0}^{b} J_{0}^{c}|(0 ; f)\rangle=\frac{2}{N} d^{a b c} \operatorname{Tr}\left(T^{a} T^{b} T^{c}\right)|(0 ; f)\rangle \\
& \rightarrow 2 i N^{2}|(0 ; f)\rangle .
\end{aligned}
$$

The first line comes from (4.17). The final nonzero contribution comes from the next 42nd-term and the following is obtained:

$$
\begin{aligned}
d^{a b c}\left(J^{a} \partial J^{b} \partial J^{c}\right)_{0}|(0 ; f)\rangle & =d^{a b c} J_{0}^{a} J_{0}^{b} J_{0}^{c}|(0 ; f)\rangle=\frac{1}{N} d^{a b c} \operatorname{Tr}\left(T^{a} T^{b} T^{c}\right)|(0 ; f)\rangle \\
& \rightarrow i N^{2}|(0 ; f)\rangle .
\end{aligned}
$$

By combining (4.21), (4.23), (4.24) and (4.25) with the corresponding coefficients in the large $N$ limit (D.4), the final zero mode eigenvalue equation can be obtained as follows:

$$
W_{0}^{(5)}|(0 ; f)\rangle=\left[-i N^{4} \times N\left(\frac{6(\lambda-2)^{2}(\lambda-1)}{\lambda^{3}(\lambda+2)}\right)-i N^{3} \times N^{2}\left(-\frac{6(\lambda-2)(\lambda-1)}{\lambda^{3}}\right)\right.
$$




$$
\begin{aligned}
& \left.+i N^{2} \times N^{3}\left(\frac{24(\lambda-1)\left(3 \lambda^{2}-20\right)}{7 \lambda^{3}(\lambda+2)}-\frac{48(\lambda-1)\left(2 \lambda^{2}-11\right)}{7 \lambda^{3}(\lambda+2)}\right)\right]|(0 ; f)\rangle \\
= & {\left[\frac{24 i(1-\lambda)(3-\lambda)(4-\lambda)}{7 \lambda^{3}(2+\lambda)}\right] N^{5}|(0 ; f)\rangle . }
\end{aligned}
$$

The same eigenvalue equation at finite $N$ and $k$ can be determined later.

\subsection{Three-point functions in the large $N$ limit}

Consider the diagonal modular invariant, by pairing up identical representations on the left (holomorphic) and right (antiholomorphic) sectors. One of the primaries is given by $(f ; 0) \otimes(f ; 0)[18]$. From the previous result (4.18), the eigenvalue of the spin-5 zero mode for $(f ; 0) \otimes(f ; 0)$ is

$$
W_{0}^{(5)}\left|\mathcal{O}_{+}\right\rangle=-\left[\frac{24 i(1+\lambda)(3+\lambda)(4+\lambda)}{7 \lambda^{3}(2-\lambda)}\right] N^{5}\left|\mathcal{O}_{+}\right\rangle, \quad \mathcal{O}_{+} \equiv(f ; 0) \otimes(f ; 0) .
$$

In addition, the eigenvalue of the spin-5 zero mode for $(0 ; f) \otimes(0 ; f)$, which is another primary, can be obtained. In this case, only four terms with no $K^{a}$ can survive because $K_{0}^{a}|(0 ; f)\rangle=0$. Therefore, the result can be calculated from (4.26) as follows:

$$
W_{0}^{(5)}\left|\mathcal{O}_{-}\right\rangle=\left[\frac{24 i(1-\lambda)(3-\lambda)(4-\lambda)}{7 \lambda^{3}(2+\lambda)}\right] N^{5}\left|\mathcal{O}_{-}\right\rangle, \quad \mathcal{O}_{-} \equiv(0 ; f) \otimes(0 ; f) .
$$

Symmetry exists between (4.27) and (4.28). One can be obtained from the other by taking $\lambda \rightarrow-\lambda$ and vice versa. Similar relations can be obtained for the other primaries $(\bar{f} ; 0) \otimes(\bar{f} ; 0)$ and $(0 ; \bar{f}) \otimes(0 ; \bar{f})$ after taking the generators of $\mathrm{SU}(N)$ in the antifundamental representation carefully,

$$
\begin{array}{ll}
W_{0}^{(5)}\left|\overline{\mathcal{O}}_{+}\right\rangle=\left[\frac{24 i(1+\lambda)(3+\lambda)(4+\lambda)}{7 \lambda^{3}(2-\lambda)}\right] N^{5}\left|\overline{\mathcal{O}}_{+}\right\rangle, & \overline{\mathcal{O}}_{+} \equiv(\bar{f} ; 0) \otimes(\bar{f} ; 0), \\
W_{0}^{(5)}\left|\overline{\mathcal{O}}_{-}\right\rangle=-\left[\frac{24 i(1-\lambda)(3-\lambda)(4-\lambda)}{7 \lambda^{3}(2+\lambda)}\right] N^{5}\left|\overline{\mathcal{O}}_{-}\right\rangle, & \overline{\mathcal{O}}_{-} \equiv(0 ; \bar{f}) \otimes(0 ; \bar{f}) .
\end{array}
$$

Compared to (4.27) and (4.28), there is an overall sign change.

The three-point functions of spin-5 current with two scalars from (4.27) and (4.28) (or (4.29)) can then be expressed as

$$
\begin{aligned}
& \left\langle\overline{\mathcal{O}}_{+} \mathcal{O}_{+} W^{(5)}\right\rangle=-\left[\frac{24 i(1+\lambda)(3+\lambda)(4+\lambda)}{7 \lambda^{3}(2-\lambda)}\right] N^{5}, \\
& \left\langle\overline{\mathcal{O}}_{-} \mathcal{O}_{-} W^{(5)}\right\rangle=\left[\frac{24 i(1-\lambda)(3-\lambda)(4-\lambda)}{7 \lambda^{3}(2+\lambda)}\right] N^{5} .
\end{aligned}
$$

The following result can be obtained by dividing these two relations (4.30) and (4.31):

$$
\frac{\left\langle\overline{\mathcal{O}}_{+} \mathcal{O}_{+} W^{(5)}\right\rangle}{\left\langle\overline{\mathcal{O}}_{-} \mathcal{O}_{-} W^{(5)}\right\rangle}=-\frac{(1+\lambda)(2+\lambda)(3+\lambda)(4+\lambda)}{(1-\lambda)(2-\lambda)(3-\lambda)(4-\lambda)} .
$$

When $\lambda=\frac{1}{2}$, this becomes -9 , which is precisely the same as the bulk computation for spin-5 in [18]. For a general $\lambda$, the bulk result in [19] for spin-5 current agrees with (4.32). 
Recall that the ratio (4.32) containing constant 1 without a minus sign is precisely the ratio for the spin-2 current, the ratio (4.32) containing constants 1 and 2 is exactly the ratio for the spin-3 current and the ratio (4.32) containing constants 1,2,3 without a minus sign is exactly the ratio for the spin- 4 current. For the spin- 5 current, the ratio of the three-point function is obtained from that of the spin- 4 current by multiplying $-\frac{(4+\lambda)}{(4-\lambda)}$. See footnote 10. The ratio of three-point function for the spin- $s$ current can be expressed as $(-1)^{s} \prod_{n=1}^{s-1} \frac{(n+\lambda)}{(n-\lambda)} \cdot{ }^{9}$ This issue for the finite $N$ and $k$ will be addressed later.

Thus far, the normalization for the spin- 5 current is not considered. The normalized spin 5 primary field $\widetilde{W}^{(5)}(z)$ can be obtained using the unnormalized spin-5 current $W^{(5)}(z)(3.15)$ as follows:

$$
\begin{aligned}
\widetilde{W}^{(5)}(z) & \equiv\left[\frac{B}{C_{33}^{4} C_{34}^{5}}\right] W^{(5)}(z), \\
B^{2} & \equiv-\frac{N}{18(N+k)^{2}(1+N)^{2}(1+k+N)^{2}(N+2)(2 k+N)(2+2 k+3 N)\left(-4+N^{2}\right)}, \\
\left(C_{34}^{5}\right)^{2} & =\frac{25(5 c+22)(N-4)[c(N+4)+3(5 N+4)(N-1)]}{(7 c+114)(N-2)[c(N+2)+(3 N+2)(N-1)]}
\end{aligned}
$$

where $B$ is the overall coefficient function of the spin-3 current [4], $C_{34}^{5}$ is the structure constant and is given in $[2,20]$. Note the factor $(N-4)$ in this expression. This suggests that the $\mathrm{SU}(4)$ coset model does not produce a spin-5 current, as mentioned before. Moreover the structure constant $C_{33}^{4}$ was given in (3.13). The structure constant $C_{34}^{5}$ can be re-expressed as follows [2]: $\left(C_{34}^{5}\right)^{2}=25\left[\frac{\left(C_{33}^{4}\right)^{2}}{\left(\hat{C}_{33}^{4}\right)^{2}}-1\right]$, where $\hat{C}_{33}^{4}$ is the structure constant for $N=4$. Therefore, for $N=4$, the structure constant vanishes. Accordingly, how does one obtain the relative coefficient factor in $\widetilde{W}^{(5)}(z)$ in (4.33)? Originally, the spin-3 current has an overall factor $B$. One can multiply $B$ at both sides in (3.12). For the spin-4 current, this study did not consider the structure constant $C_{33}^{4}$ in [7] in front of $W^{(4)}(w)$ of the second order pole in the $\mathrm{OPE} W^{(3)}(z) W^{(3)}(w)$. This analysis checked that for $N=4$, the spin-4 current $\frac{W^{(4)}(z)}{C_{33}^{4}}$ provides the highest singular term, $\frac{c}{4}$, correctly. This suggests that one should divide out the structure constant $C_{33}^{4}$ in $(3.12) \cdot{ }^{10} \frac{B}{C_{33}^{4}} W^{(5)}(w)$ is on the right hand side. Once again, this quantity should be multiplied by the structure constant

\footnotetext{
${ }^{9}$ More explicitly, one can use the equations (4.51) and (4.53) of [19] to check the bulk result corresponding to (4.32). What does this imply in the bulk theory side? The three-point function in the bulk theory can be determined from the asymptotic behavior of a scalar field in $A d S_{3}$ as the radial coordinate goes to infinity. On the other hand, the CFT result of this paper (i.e. the equation (4.32)) indicates that for fixed spin $s=5$, the $\lambda$-dependent part of this scalar field asymptotics has very simple factorized forms $\pm \prod_{n=1}^{4}(n \pm \lambda)$. This provides the nontrivial information on the change in the $A d S_{3}$ scalar under the gauge transformation for the higher spin deformation in the bulk theory. Of course, this feature arises in section 4 of [19] via bulk theory computation. Therefore, one might think that the physical implication for the three-point function (4.32) is to indicate a hidden functional dependence of the change in the $A d S_{3}$ scalar (under the gauge transformation) on the deformation parameter $\lambda$ where the $\lambda=\frac{1}{2}$ in undeformed bulk theory.

${ }^{10}$ The eigenvalue equations can be calculated for the other currents. For the spin- 4 current, the large $N$ limit was given in (3.47) of [7]. By dividing $C_{33}^{4}$ with a large $N$ limit,
}

$$
\widetilde{W}_{0}^{(4)}\left|\mathcal{O}_{+}\right\rangle=\frac{\sqrt{5}}{20}(1+\lambda) \sqrt{\frac{(2+\lambda)(3+\lambda)}{(2-\lambda)(3-\lambda)}}\left|\mathcal{O}_{+}\right\rangle, \widetilde{W}_{0}^{(4)}\left|\mathcal{O}_{-}\right\rangle=\frac{\sqrt{5}}{20}(1-\lambda) \sqrt{\frac{(2-\lambda)(3-\lambda)}{(2+\lambda)(3+\lambda)}}\left|\mathcal{O}_{-}\right\rangle
$$


$C_{34}^{5}$ and divide it out. This leaves the quantity $\frac{B}{C_{33}^{4} C_{34}^{5}} W^{(5)}(w)$, which is denoted by $\widetilde{W}^{(5)}(w)$. The central charge term of the OPE between $\widetilde{W}^{(5)}$ and itself will have the form

$$
\widetilde{W}^{(5)}(z) \widetilde{W}^{(5)}(w)=\frac{1}{(z-w)^{10}} \frac{c}{5}+\cdots
$$

In this normalization (4.37), the previous eigenvalue equations (4.27) and (4.28) become

$$
\begin{aligned}
& \widetilde{W}_{0}^{(5)}\left|\mathcal{O}_{+}\right\rangle=\frac{\sqrt{14}}{70}(1+\lambda) \sqrt{\frac{(2+\lambda)(3+\lambda)(4+\lambda)}{(2-\lambda)(3-\lambda)(4-\lambda)}}\left|\mathcal{O}_{+}\right\rangle, \\
& \widetilde{W}_{0}^{(5)}\left|\mathcal{O}_{-}\right\rangle=-\frac{\sqrt{14}}{70}(1-\lambda) \sqrt{\frac{(2-\lambda)(3-\lambda)(4-\lambda)}{(2+\lambda)(3+\lambda)(4+\lambda)}}\left|\mathcal{O}_{-}\right\rangle .
\end{aligned}
$$

From the description of footnote 10, the general $\lambda$ dependent behavior for the eigenvalue equations of a higher spin- $s$ current can be read off and

$$
\begin{aligned}
& \widetilde{W}_{0}^{(s)}\left|\mathcal{O}_{+}\right\rangle=\left[(1+\lambda) \sqrt{\frac{(2+\lambda)(3+\lambda) \cdots(s-1+\lambda)}{(2-\lambda)(3-\lambda) \cdots(s-1-\lambda)}}\right]\left|\mathcal{O}_{+}\right\rangle \\
& \widetilde{W}_{0}^{(s)}\left|\mathcal{O}_{-}\right\rangle=(-1)^{s}\left[(1-\lambda) \sqrt{\frac{(2-\lambda)(3-\lambda) \cdots(s-1-\lambda)}{(2+\lambda)(3+\lambda) \cdots(s-1+\lambda)}}\right]\left|\mathcal{O}_{-}\right\rangle .
\end{aligned}
$$

In (4.39), the second equation can be obtained from the first equation by taking $\lambda \rightarrow-\lambda$ and vice versa up to an overall sign.

Note that the ratio of unnormalized spin-5 and normalized one in the large $N$ limit is

$$
\frac{W^{(5)}(z)}{\widetilde{W}^{(5)}(z)}=\frac{C_{33}^{4} C_{34}^{5}}{B} \rightarrow-i N^{5} \frac{120 \sqrt{14}}{7 \lambda^{3}} \sqrt{\frac{(3-\lambda)(3+\lambda)(4-\lambda)(4+\lambda)}{(2-\lambda)(2+\lambda)}}
$$

\subsection{Eigenvalue equations and three-point functions at a finite $(N, k)$}

At a finite $N$ and $k$, the 52 coefficient functions are given in terms of $(N, k)$ in (D.1) and the identities (4.10)-(4.15) hold for any $N$. The exact zero mode eigenvalue equations can be obtained. Surprisingly, the eigenvalue equations are simple factorized forms.

For the spin-3 current, by multiplying (4.22) or (4.20) of [13] by the constant $A_{1}$ (3.8) and constant $B(4.33)$ with a large $N$ limit, respectively, results in similar equations as follows:

$$
\widetilde{W}_{0}^{(3)}\left|\mathcal{O}_{+}\right\rangle=\frac{\sqrt{2}}{6}(1+\lambda) \sqrt{\frac{(2+\lambda)}{(2-\lambda)}}\left|\mathcal{O}_{+}\right\rangle, \quad \widetilde{W}_{0}^{(3)}\left|\mathcal{O}_{-}\right\rangle=-\frac{\sqrt{2}}{6}(1-\lambda) \sqrt{\frac{(2-\lambda)}{(2+\lambda)}}\left|\mathcal{O}_{-}\right\rangle .
$$

The $\lambda$-dependent part of the eigenvalue equation of spin-4 current in (4.34) can be obtained from that of the spin-3 current in (4.35) by multiplying $\sqrt{\frac{(3+\lambda)}{(3-\lambda)}}$ and $\sqrt{\frac{(3-\lambda)}{(3+\lambda)}}$, respectively, up to the sign. This feature holds for the spin-3 and spin-2 currents. That is, by multiplying $\sqrt{\frac{(2+\lambda)}{(2-\lambda)}}$ and $\sqrt{\frac{(2-\lambda)}{(2+\lambda)}}$ with the following spin-2 eigenvalue equations,

$$
T_{0}\left|\mathcal{O}_{+}\right\rangle=\frac{1}{2}(1+\lambda)\left|\mathcal{O}_{+}\right\rangle, \quad T_{0}\left|\mathcal{O}_{-}\right\rangle=\frac{1}{2}(1-\lambda)\left|\mathcal{O}_{-}\right\rangle,
$$

one obtains the above eigenvalue equations (4.35) up to the sign. 
For the primary state, $|(f ; 0)\rangle$, the 48 terms contribute to the final result. The traces corresponding to the $28 \mathrm{th}, 32 \mathrm{nd}, 34 \mathrm{th}$, and $38 \mathrm{th}$ terms of the spin- 5 current are identically zero. The eigenvalue equation can be expressed as follows:

$$
\begin{aligned}
W_{0}^{(5)}|(f ; 0)\rangle= & {\left[-24 i(k+1)(N-4)(N-3)(N-1)(N+1)^{2}(N+2)(k+N+1)(k+2 N)\right.} \\
& (k+2 N+1)(3 k+4 N+3)(4 k+5 N+4) /\left(N ^ { 3 } ( 2 k + N ) \left(7 k^{2} N+107 k^{2}+14 k N^{2}\right.\right. \\
& \left.\left.\left.+221 k N+107 k+114 N^{2}+114 N\right)\right)\right]|(f ; 0)\rangle .
\end{aligned}
$$

Note the factor $(N-4)(N-3)$ in the numerator of $(4.41)$. $(N-s+1)(N-s+2) \cdots(N-$ $4)(N-3)$ is expected in the general spin- $s$ eigenvalue equation. Of course, the large $N$ limit result (4.18) can be reproduced from the more general result (4.41).

For the other primary state $|(0 ; f)\rangle$, the eigenvalue equation becomes

$$
\begin{aligned}
W_{0}^{(5)}|(0 ; f)\rangle= & {\left[24 i k(k+1)(N-4)(N-3)(N-1)(N+1)^{2}(N+2)(k+N)(k+2 N)(3 k+2 N)\right.} \\
& (4 k+3 N) /\left(N ^ { 3 } ( 2 k + 3 N + 2 ) \left(7 k^{2} N+107 k^{2}+14 k N^{2}+221 k N+107 k+114 N^{2}\right.\right. \\
& +114 N))]|(0 ; f)\rangle .
\end{aligned}
$$

The factor, $(N-4)(N-3)$, in (4.42) appears in this case and the large $N$ limit (4.26) can be obtained from the more general result (4.42). The three-point functions from (4.41) and $(4.42)$ can be summarized as

$$
\begin{aligned}
\left\langle\overline{\mathcal{O}}_{+} \mathcal{O}_{+} W^{(5)}\right\rangle= & {\left[-24 i(k+1)(N-4)(N-3)(N-1)(N+1)^{2}(N+2)(k+N+1)(k+2 N)\right.} \\
& (k+2 N+1)(3 k+4 N+3)(4 k+5 N+4) /\left(N ^ { 3 } ( 2 k + N ) \left(7 k^{2} N+107 k^{2}+14 k N^{2}\right.\right. \\
& \left.\left.\left.+221 k N+107 k+114 N^{2}+114 N\right)\right)\right], \\
\left\langle\overline{\mathcal{O}}_{-} \mathcal{O}_{-} W^{(5)}\right\rangle= & {\left[24 i k(k+1)(N-4)(N-3)(N-1)(N+1)^{2}(N+2)(k+N)(k+2 N)(3 k+2 N)\right.} \\
& (4 k+3 N) /\left(N ^ { 3 } ( 2 k + 3 N + 2 ) \left(7 k^{2} N+107 k^{2}+14 k N^{2}+221 k N+107 k+114 N^{2}\right.\right. \\
& +114 N))] .
\end{aligned}
$$

Obviously, the previous results (4.30) and (4.31) can be obtained from (4.43) with the appropriate limit.

From (4.41) and (4.42) or (4.43), the ratio between the three-point functions at a finite $N$ and $k$, can be expressed as

$$
\frac{\left\langle\overline{\mathcal{O}}_{+} \mathcal{O}_{+} W^{(5)}\right\rangle}{\left\langle\overline{\mathcal{O}}_{-} \mathcal{O}_{-} W^{(5)}\right\rangle}=-\frac{(k+N+1)(k+2 N+1)(2 k+3 N+2)(3 k+4 N+3)(4 k+5 N+4)}{k(k+N)(2 k+N)(3 k+2 N)(4 k+3 N)} .
$$

Of course, the large $N$ behavior in (4.32) can be observed from this general expression by taking the appropriate limit as before. In other words, (4.32) is obtained once the numerical constants, 1,1,2,3,4 in the numerator of (4.44) are ignored. This result comes from the $W_{N}$ coset CFT at a finite $N$ and $k$, and corresponds to the ratio of three-point functions in the deformed $A d S_{3}$ bulk theory. ${ }^{11}$

\footnotetext{
${ }^{11}$ For the lower spin currents, the eigenvalues and three-point functions can also be analyzed. For the spin-4 current (A.1) or (3.9), the following result is described in appendix F (F.25)

$$
\begin{aligned}
& W_{0}^{(4)}|(f ; 0)\rangle=\left[\frac{2(k+1)(N-3)\left(N^{2}-1\right)(k+2 N)(k+2 N+1)(3 k+4 N+3)}{N^{2}(k+N)(2 k+N) d(N, k)}\right]|(f ; 0)\rangle, \\
& W_{0}^{(4)}|(0 ; f)\rangle=\left[\frac{2 k(k+1)(N-3)\left(N^{2}-1\right)(k+2 N)(3 k+2 N)}{N^{2}(k+N+1)(2 k+3 N+2) d(N, k)}\right]|(0 ; f)\rangle,
\end{aligned}
$$

where $d(N, k) \equiv 17 k+17 k^{2}+22 N+39 k N+5 k^{2} N+22 N^{2}+10 k N^{2}$ is introduced. Note the presence of
} 
In $[2,5]$, the minimal representation of $\mathcal{W}_{\infty}[\mu]$, where the fewest number of low lying states exists, was studied. Indirectly, the eigenvalue of the zero mode of the spin- 5 current, $x$, can be expressed in terms of the spin (or conformal dimension), $h$, the eigenvalue of spin3 current, $w$, the eigenvalue of spin- 4 current, $u$ (note that four times their spin- 4 field is equal to the spin- 4 current in the present study and see (A.22) of [2]), the central charge $c$, the constant, $N_{3}$, and the structure constant, $N_{4}=\frac{75}{16 \cdot 56} N_{3}^{2}\left(C_{33}^{4}\right)^{2}$, which depends on $(c, \mu)$. Explicitly, equations $(B .8),(B .15),(B .16)$ and (B.17) of [2] (where the version 2 of arXiv corrected some typographical errors that appeared in the version 1 of arXiv) contain the eigenvalue of the zero mode of the spin- 5 current, $x$. By substituting the following quantities

$$
\begin{aligned}
N_{4} & =\frac{6(1+k)(-3+N)(1+N)(k+2 N)(3 k+2 N)(3+3 k+4 N)}{7(-2+N)(2 k+N)(2+2 k+3 N) d(N, k)}, \\
d(N, k) & \equiv\left(17 k+17 k^{2}+22 N+39 k N+5 k^{2} N+22 N^{2}+10 k N^{2}\right), \\
N_{3} & =\frac{2}{5}, \\
h(f ; 0) & =\frac{(N-1)(k+2 N+1)}{2 N(k+N)}, \\
w(f ; 0) & =-\frac{i}{N^{2}}\left(N^{2}-4\right)\left(N^{2}-1\right)(k+N+1)(k+2 N+1)(2 k+3 N+2), \\
u(f ; 0) & =\frac{2(k+1)(N-3)\left(N^{2}-1\right)(k+2 N)(k+2 N+1)(3 k+4 N+3)}{N^{2}(k+N)(2 k+N) d(N, k)}, \\
c & =\frac{k(-1+N)(1+k+2 N)}{(k+N)(1+k+N)},
\end{aligned}
$$

into the formula $(B .15)$ of $[2]$

$$
20 \times w\left[\frac{4 N_{4}}{25 N_{3}}-\frac{208 N_{4}}{25 N_{3}\left(c+\frac{114}{7}\right)}\left(h+\frac{3}{7}\right)+\frac{1}{4} \times \frac{6 u}{5 h}\right],
$$

the eigenvalue given in (4.41) can be derived. Note that $B$ times a spin-5 current is equal to $4 \times 5(=20)$ times their spin-5 field. This is why the extra numerical factor 20 is placed

the factor $(N-3)$. All the terms in (A.1) or (3.9) contribute to the first eigenvalue, whereas three terms in (A.1) (or four terms in (3.9)) can survive in the second eigenvalue in (4.45). For the spin-3 current (3.7), the two eigenvalue equations from appendix $F($ F.26) are

$$
\begin{aligned}
W_{0}^{(3)}|(f ; 0)\rangle & =-\left[\frac{i}{N^{2}}\left(N^{2}-4\right)\left(N^{2}-1\right)(k+N+1)(k+2 N+1)(2 k+3 N+2)\right]|(f ; 0)\rangle, \\
W_{0}^{(3)}|(0 ; f)\rangle & =\left[\frac{i}{N^{2}} k\left(N^{2}-4\right)\left(N^{2}-1\right)(k+N)(2 k+N)\right]|(0 ; f)\rangle .
\end{aligned}
$$

The first term of (3.7) can contribute to the second eigenvalue of (4.46). Note the factor $(N-2)$ in (4.46). Finally, the spin-2 current (2.6) has the following eigenvalue equations with appendix $F$ (F.27)

$$
T_{0}|(f ; 0)\rangle=\left[\frac{(N-1)(k+2 N+1)}{2 N(k+N)}\right]|(f ; 0)\rangle, \quad T_{0}|(0 ; f)\rangle=\left[\frac{k(N-1)}{2 N(k+N+1)}\right]|(0 ; f)\rangle .
$$

The eigenvalue equations for the general spin- $s$ are difficult to obtain from (4.41), (4.42), (4.45), (4.46), and (4.47). 
in (4.49). In addition, the eigenvalues in the expressions (4.45), (4.46) and (4.47) are substituted in (4.48). Similarly, using the following eigenvalues with the other quantities in (4.48)

$$
\begin{aligned}
h(0 ; f) & =\frac{k(N-1)}{2 N(k+N+1)}, \\
w(0 ; f) & =\frac{i}{N^{2}} k\left(N^{2}-4\right)\left(N^{2}-1\right)(k+N)(2 k+N), \\
u(0 ; f) & =\frac{2 k(k+1)(N-3)\left(N^{2}-1\right)(k+2 N)(3 k+2 N)}{N^{2}(k+N+1)(2 k+3 N+2) d(N, k)},
\end{aligned}
$$

the expression (4.49) leads to the other eigenvalue in (4.42). Checking that the eigenvalues for the spin-2,3, 4 fields written in terms of the conformal dimension, $h$, and the central charge $c$ in [2] coincide with those expressed as $N$ and $k$ in (4.48) and (4.50) is quite simple.

The following ratios between the three-point functions for spin $s=2,3,4$ can be obtained from (4.47), (4.46), and (4.45)

$$
\begin{aligned}
\frac{\left\langle\overline{\mathcal{O}}_{+} \mathcal{O}_{+} T\right\rangle}{\left\langle\overline{\mathcal{O}}_{-} \mathcal{O}_{-} T\right\rangle} & =\frac{(k+N+1)(k+2 N+1)}{k(k+N)}, \\
\frac{\left\langle\overline{\mathcal{O}}_{+} \mathcal{O}_{+} W^{(3)}\right\rangle}{\left\langle\overline{\mathcal{O}}_{-} \mathcal{O}_{-} W^{(3)}\right\rangle} & =-\frac{(k+N+1)(k+2 N+1)(2 k+3 N+2)}{k(k+N)(2 k+N)}, \\
\frac{\left\langle\overline{\mathcal{O}}_{+} \mathcal{O}_{+} W^{(4)}\right\rangle}{\left\langle\overline{\mathcal{O}} \_\mathcal{O}_{-} W^{(4)}\right\rangle} & =\frac{(k+N+1)(k+2 N+1)(2 k+3 N+2)(3 k+4 N+3)}{k(k+N)(2 k+N)(3 k+2 N)} .
\end{aligned}
$$

At $s=5$, equation (4.44) can be derived. As a spin $s$ increases, the extra factors in the numerator and denominator appear up to the sign. This can be generalized to the arbitrary spin- $s$ current, $W^{(s)}$, whose ratio is expressed as

$$
\frac{\left\langle\overline{\mathcal{O}}_{+} \mathcal{O}_{+} W^{(s)}\right\rangle}{\left\langle\overline{\mathcal{O}}_{-} \mathcal{O}_{-} W^{(s)}\right\rangle}=(-1)^{s} \frac{(k+N+1)}{(k+N)} \prod_{n=1}^{s-1}\left[\frac{n k+(n+1) N+n}{n k+(n-1) N}\right] .
$$

Of course, this generalizes the fixed $\lambda=\frac{1}{2}$ case in bulk theory [18] and the arbitrary $\lambda$ in the boundary/bulk theory [19] to the finite $N$ and $k$. In other words, for fixed spin $s$, each factor in the numerator of (4.52) has the extra numerical constants, $1,1,2,3,4, \cdots,(s-1)$. The previous results in [19], where they assumed $\mathcal{W}_{\infty}[\lambda]$ symmetry in the CFT computations, can be obtained if these constants are ignored.

\subsection{More general coset model}

The more general coset model, $\frac{G}{H}$, can be described by two arbitrary levels $\left(k_{1}, k_{2}\right)$

$$
\frac{G}{H}=\frac{\widehat{S U}(N)_{k_{2}} \oplus \widehat{S U}(N)_{k_{1}}}{\widehat{S U}(N)_{k_{2}+k_{1}}}
$$

Many additional extra currents can be expected in the general coset model (4.53). The corresponding algebra should be larger than the conventional $W_{N}$ algebra. For example, the explicit form of the structure constant, $C_{33}^{4}$, is not known so far in the general coset 
model. Recall that the spin-3 and spin- 4 currents with arbitrary levels $\left(k_{1}, k_{2}\right)$ are known in the general coset model. If the central charge term of the OPE between the spin- 4 current with itself (which is equal to $\frac{c}{4}$ with the central charge in the general coset model) is calculated, the explicit form for the structure constant, $C_{33}^{4}$, in the general coset model can be determined completely.

The explicit forms of $C_{33}^{4}$ for some fixed $N$ from the Thielemans package [17] are presented. The left hand side of (3.12) is known for an arbitrary $\left(k_{1}, k_{2}\right)$ with a fixed $N=4,5$, and two arbitrary parameters in front of $\eta_{\Lambda}$ and $\eta_{W}$ are introduced. The spin-5 current can be written as in (3.14) with two parameters. Now the OPE between $T(z)$ and the spin- 5 current can be calculated by requiring the primary condition. The above two parameters can be fixed completely and the structure constants for a fixed $N=4,5$ with arbitrary levels are as follows:

$$
\begin{aligned}
{\left[C_{33}^{4}\left(N=5, k_{1}, k_{2}\right)\right]^{2}=} & {\left[6 4 \left(k_{1}^{4}\left(2771 k_{2}^{2}+10530 k_{2}+2575\right)+k_{1}^{3}\left(5542 k_{2}^{3}+76480 k_{2}^{2}\right.\right.\right.} \\
& \left.+215750 k_{2}+51500\right)+k_{1}^{2}\left(2771 k_{2}^{4}+76480 k_{2}^{3}+600725 k_{2}^{2}+1399250 k_{2}\right. \\
& +406250)+10 k_{1}\left(1053 k_{2}^{4}+21575 k_{2}^{3}+139925 k_{2}^{2}+309625 k_{2}+148750\right) \\
& \left.+25\left(k_{2}+5\right)^{2}\left(103 k_{2}^{2}+1030 k_{2}+3375\right)\right] /\left[21\left(2 k_{1}+5\right)\left(2 k_{2}+5\right)\right. \\
& \times\left(2 k_{1}^{3}\left(71 k_{2}+55\right)+k_{1}^{2}\left(284 k_{2}^{2}+2705 k_{2}+1925\right)+k_{1}\left(142 k_{2}^{3}+2705 k_{2}^{2}\right.\right. \\
& \left.\left.\left.+13675 k_{2}+11000\right)+55\left(k_{2}+5\right)^{2}\left(2 k_{2}+15\right)\right)\right] \\
{\left[C_{33}^{4}\left(N=4, k_{1}, k_{2}\right)\right]^{2}=} & 8\left(k_{1}^{4}\left(409 k_{2}^{2}+1296 k_{2}+320\right)+k_{1}^{3}\left(818 k_{2}^{3}+9136 k_{2}^{2}+21376 k_{2}\right.\right. \\
& +5120)+k_{1}^{2}\left(409 k_{2}^{4}+9136 k_{2}^{3}+58240 k_{2}^{2}+112976 k_{2}+33664\right) \\
& +16 k_{1}\left(81 k_{2}^{4}+1336 k_{2}^{3}+7061 k_{2}^{2}+13280 k_{2}+6592\right) \\
& \left.\left.+64\left(k_{2}+4\right)^{2}\left(5 k_{2}^{2}+40 k_{2}+126\right)\right)\right] /\left[3 ( k _ { 1 } + 2 ) ( k _ { 2 } + 2 ) \left(k_{1}^{3}\left(97 k_{2}+88\right)\right.\right. \\
& +2 k_{1}^{2}\left(97 k_{2}^{2}+767 k_{2}+616\right)+k_{1}\left(97 k_{2}^{3}+1534 k_{2}^{2}+6592 k_{2}+5632\right) \\
& \left.\left.+88\left(k_{2}+4\right)^{2}\left(k_{2}+6\right)\right)\right] .
\end{aligned}
$$

These structure constants are reduced to the structure constants of the minimal model when $k_{1}$ is fixed to 1 (Of course, $k_{2}=k$ ). Furthermore, the highest singular term of the spin-4 current (when $N=4$ ) was checked to ensure that it behaves correctly with (4.54).

\section{Conclusions and outlook}

The spin-5 Casimir operator (3.15) was obtained by calculating the second-order pole in the OPE between the spin-3 Casimir operator (3.7) and the spin-4 Casimir operator (3.9). The three-point functions with two scalars (4.30) and (4.31) for all values of the 't Hooft coupling in the large $N$ limit (and without any limit) were obtained by analyzing the zero-mode eigenvalue equations carefully. These three-point functions (4.43) were dual to those in the $A d S_{3}$ higher spin gravity theory with matter. A future study should calculate the corresponding three-point functions in the bulk theory.

- Although the spin-5 current contains the multiple product between the $d, f$ symbol of rank 3 , or the $\delta$ symbol of rank 2 , one does not observe a completely symmetric traceless $d$ symbol of rank 5. A future study should examine the generalization of (A.3) to a higher rank. From the second order pole of the OPE between the spin- 3 current and the spin- $s$ 
current, where $s \geq 4$, one can imagine that a higher $d$ symbol of rank $s$ can be written in terms of the multiple product of $d$ symbol of rank 3 , where the number of $d$ symbols is $(s-2)$. The generalization of [21] to the multiple product of $d$ symbols is needed.

- Because the spin-4 current is known for any arbitrary level $\left(k_{1}, k_{2}\right)$, it would be interesting to calculate the eighth-order pole of the OPE between the spin- 4 current and itself, as mentioned before. Once this is done, the structure constant $C_{33}^{4}$ in the general coset model can then be obtained. Of course, this structure constant will reduce to the previous result (3.13) in the coset minimal model (1.1) when $k_{1}=1$ and $k_{2}=k$. Furthermore, for $N=4$ or $N=5$, one should obtain the relations (4.54).

- What of the spin-6 current? From the vacuum character computation described before, there are eleven nonprimary spin- 6 currents that can be constructed from the spin$2,3,4,5$ currents (with some derivatives) and a single primary spin-6 current. This current might be constructed by calculating the OPE between the spin- 3 current (3.7) and spin- 5 current (3.15). Definitely, the independent terms will be greater than 52 terms for the spin-5 current. At least, relation (4.52) for $s=6$ can be observed. Furthermore, because of this relation, one also expects that the eigenvalue equations for two coset primary states has factorized forms, as in the other lower higher spin cases. In a bulk theory point of view, the asymptotic quantum symmetry algebra $\mathcal{W}_{\infty}[\mu]$ in $[2]$ should be extended to the algebra containing the commutator between the spin-3 Laurent mode and the spin-5 Laurent mode. The previous work given in [22] (classical algebra) is expected to be helpful in obtaining this commutator. When $N=6$, the above construction should be related to the work reported elsewhere [23], where $W_{6}$ algebra was studied.

- Because the spin-3, 4, 5 Casimir currents are known for the coset model (1.1), one can also check whether the other structure constants, $C_{44}^{4}, C_{45}^{5}$ in [20], can be expressed in terms of $C_{33}^{4}$ (3.13). The former can be obtained from the highest singular term in the OPE of the spin- 4 current and itself and the latter can be obtained from the OPE of the spin- 4 current and the spin- 5 current as well as the OPE between the spin- 5 current and itself. In principle, the other OPEs, spin-3 spin-5, spin-4 spin-4, spin-4 spin-5, and spin- 5 spin- 5 , can be obtained and the algebra should be related to the $W_{5}$ algebra [23], where $N$ is fixed to $N=5$. Extracting several quasi-primary fields in these OPEs is nontrivial. For example, the OPE between the spin- 5 current and itself provides the second order pole, where the possible quasi-primary fields of spin- 8 arise. Even in the well-known quantum Miura transformations [3], the formula appears quite complicated. As mentioned in the introduction, the general procedure to obtain the correct quasi-primaries is based on previous studies [8-12].

- The $\mathcal{N}=1$ supersymmetric coset model can be obtained by taking one of the levels to be $N$ in the context of that reported elsewhere [12, 24, 25]. The immediate question in this direction is what are the spin contents of this coset model? A future study should examine this particular coset model because this might be the possible dual theory for the $A d S_{3}$ string theory. Determining the $N$-generalization for the character technique is an open problem.

- The duality in $[1,2]$ has been generalized to the duality with the other group, which is analogous to the $O(N)$ vector model in one dimension higher [26, 27]. In [28, 29], the explicit Casimir currents are constructed. In contrast to the present work, the Casimir 
construction with orthogonal group has the symmetric $\mathrm{SO}(N)$ invariant tensor of rank 2. In other words, the general $N$-dependence in the OPEs can be read off from the fixed several $N$ case results. No complicated contractions were observed between the $d$ symbols in the $\mathrm{SU}(N)$ coset model.

- The large $\mathcal{N}=4$ holography was found recently in [30]. The 2-dimensional CFT has many supersymmetries. Sometimes it is useful for describing the explicit construction of Casimir operators in $\mathcal{N}=2$ superspace. For example, in the context of the $\mathcal{N}=2$ KazamaSuzuki model. Some relevant descriptions can be found in [31, 32]. Thus far, it is unknown how to construct the $\mathcal{N}=4$ extension of $W_{3}$ algebra. Among the sixteen currents (in the spin contents, they are given by four spin- $\frac{1}{2}$ currents, seven spin- 1 currents, four spin- $\frac{3}{2}$ currents and a spin-2 current) in this large $\mathcal{N}=4$ superconformal algebra, only eleven currents, after projecting out four spin- $\frac{1}{2}$ currents and a spin- 1 current, play an important role in higher spin theory. The possible lowest higher spin current contains the following spin contents $\left(1, \frac{3}{2}, \frac{3}{2}, 2\right),\left(\frac{3}{2}, 2,2, \frac{5}{2}\right),\left(\frac{3}{2}, 2,2, \frac{5}{2}\right)$ and $\left(2, \frac{5}{2}, \frac{5}{2}, 3\right)$ in $\mathcal{N}=2$ superspace notation. In a future study, it would be very interesting to construct the above eleven currents living in large $\mathcal{N}=4$ nonlinear algebra and the above sixteen currents that act as extra higher spin currents in the specific coset model suggested in [30]. Therefore, it would be interesting to find $(11+16)$ currents explicitly in terms of the coset WZW primaries.

\section{Acknowledgments}

CA would like to thank M.R. Gaberdiel for discussions. This work was supported by the Mid-career Researcher Program through the National Research Foundation of Korea (NRF) grant funded by the Korean government (MEST) (No. 2012-045385). This research was supported by Kyungpook National University Research Fund 2012. CA acknowledges warm hospitality from the School of Liberal Arts (and Institute of Convergence Fundamental Studies), Seoul National University of Science and Technology.

\section{A Coefficient functions appearing in the spin-4 current}

The spin- 4 current can be obtained by calculating the second-order pole in the OPE between the spin- 3 current and itself. In this calculation, the quasi-primary field of spin- 4 and the descendant term of stress energy tensor of spin- 4 with the correct coefficients should be subtracted from the above second-order pole. The coset spin-4 primary field is given by [7]

$$
\begin{aligned}
W^{(4)}(z)= & d^{a b c d}\left(c_{1} J^{a} J^{b} J^{c} J^{d}+c_{2} J^{a} J^{b} J^{c} K^{d}+c_{3} J^{a} J^{b} K^{c} K^{d}+c_{4} J^{a} K^{b} K^{c} K^{d}+c_{5} K^{a} K^{b} K^{c} K^{d}\right)(z) \\
& +d^{a b e} d^{c d e}\left(c_{6} J^{a} J^{b} J^{c} J^{d}+c_{7} J^{a} J^{b} J^{c} K^{d}+c_{8} J^{a} J^{b} K^{c} K^{d}+c_{9} J^{a} K^{b} K^{c} K^{d}+c_{10} K^{a} K^{b} K^{c} K^{d}\right)(z) \\
& +c_{11} J^{a} J^{a} J^{b} J^{b}(z)+c_{12} J^{a} J^{a} J^{b} K^{b}(z)+c_{13} J^{a} J^{a} K^{b} K^{b}(z)+c_{14} J^{a} K^{a} K^{b} K^{b}(z) \\
& +c_{15} K^{a} K^{a} K^{b} K^{b}(z)+c_{17} \partial^{2} J^{a} K^{a}(z)+c_{20} \partial J^{a} \partial K^{a}(z)+c_{22} J^{a} \partial^{2} K^{a}(z) \\
& +c_{23} J^{a} J^{b} K^{a} K^{b}(z),
\end{aligned}
$$

where the coefficient functions $c_{i}$ are given in [7] or in appendix $A$ (A.5)-(A.23). As the OPE between the spin-3 current (3.7) and spin- 4 current (A.1) is calculated, the identity between 
the $d$ symbols should be used. Therefore, it is better to rewrite the above spin- 4 current using the $d$ symbol of rank 3 . The various identities of these $d$ symbols of rank 3 can be used.

Using the identity [7]

$$
\begin{aligned}
d^{a b c d} J^{a} J^{b} J^{c} J^{d}(z)= & 3 d^{a b e} d^{c d e} J^{a} J^{b} J^{c} J^{d}(z)-\frac{12\left(N^{2}-4\right)}{N\left(N^{2}+1\right)} J^{a} J^{a} J^{b} J^{b}(z) \\
& -\frac{3\left(N^{2}-4\right)\left(N^{2}-3\right)}{N^{2}+1} \partial J^{a} \partial J^{a}(z)+\frac{2\left(N^{2}-4\right)\left(N^{2}-3\right)}{N^{2}+1} \partial^{2} J^{a} J^{a}(z),
\end{aligned}
$$

the first term of (A.1) can be decomposed into the right hand side of (A.2), where the following identity is used [33]

$$
d^{a b c d}=d^{a b e} d^{e c d}+d^{a c e} d^{e b d}+d^{a d e} d^{e b c}-\frac{4\left(N^{2}-4\right)}{N\left(N^{2}+1\right)}\left(\delta^{a b} \delta^{c d}+\delta^{a c} \delta^{b d}+\delta^{a d} \delta^{b c}\right) .
$$

The first term of (A.1) can be absorbed into the sixth- and eleventh-terms of (A.1) besides the derivative terms.

One can calculate similar identities as follows [7]:

$$
\begin{aligned}
d^{a b c d} J^{a} J^{b} J^{c} K^{d}(z)= & 3 d^{a b e} d^{c d e} J^{a} J^{b} J^{c} K^{d}(z)-\frac{12\left(N^{2}-4\right)}{N\left(N^{2}+1\right)} J^{a} J^{a} J^{b} K^{b}(z) \\
& -\frac{3\left(N^{2}-4\right)\left(N^{2}-3\right)}{N\left(N^{2}+1\right)} f^{a b c} \partial J^{a} J^{b} K^{c}(z)+\frac{2\left(N^{2}-4\right)\left(N^{2}-3\right)}{N^{2}+1} \partial^{2} J^{a} K^{a}(z), \\
d^{a b c d} J^{a} J^{b} K^{c} K^{d}(z)= & d^{a b e} d^{c d e} J^{a} J^{b} K^{c} K^{d}(z)+2 d^{a c e} d^{b d e} J^{a} J^{b} K^{c} K^{d}(z) \\
& -\frac{4\left(N^{2}-4\right)}{N\left(N^{2}+1\right)} J^{a} J^{a} K^{b} K^{b}(z)-\frac{8\left(N^{2}-4\right)}{N\left(N^{2}+1\right)} J^{a} J^{b} K^{a} K^{b}(z) \\
& -\frac{\left(N^{2}-4\right)\left(N^{2}-3\right)}{N^{2}+1} \partial J^{a} \partial K^{a}(z), \\
d^{a b c d} J^{a} K^{b} K^{c} K^{d}(z)= & 3 d^{a b e} d^{c d e} J^{a} K^{b} K^{c} K^{d}(z)-\frac{12\left(N^{2}-4\right)}{N\left(N^{2}+1\right)} J^{a} K^{a} K^{b} K^{b}(z) \\
& +\frac{3\left(N^{2}-4\right)\left(N^{2}-3\right)}{N\left(N^{2}+1\right)} f^{a b c} \partial K^{a} K^{b} J^{c}(z)-\frac{\left(N^{2}-4\right)\left(N^{2}-3\right)}{N^{2}+1} J^{a} \partial^{2} K^{a}(z), \\
d^{a b c d} K^{a} K^{b} K^{c} K^{d}(z)= & d^{a b c d} J^{a} J^{b} J^{c} J^{d}(z) \mid J^{a} \leftrightarrow K^{a} .
\end{aligned}
$$

Checking whether the second term of (A.1) can be absorbed into the seventh- and twelfthterms of (A.1) is relatively simple. Furthermore, the $f$ symbol term (due to the normal ordering of the currents $J^{a}(z)$ and $\left.K^{a}(z)\right)$ in the first equation of (A.4) newly arises. The third term of (A.1) can contribute to the eighth- and thirteenth-terms and the two new independent terms appear. Similarly, the fourth-term of (A.1) can be absorbed into the ninth- and fourteenth-terms. In this case, the $f$ symbol term also arises. The symmetry between the spin- 1 currents is used at the final equation of (A.4). That is, the left hand side of that equation can be obtained from (A.2) by changing $J^{a}(z)$ to $K^{a}(z)$ and vice versa.

The following presents the various coefficient functions in (A.1) that appeared in [7]:

$$
\begin{aligned}
c_{1}= & {\left[-k_{2}\left(N^{2}+1\right)\left(k_{1}^{2} k_{2} N^{2}-3 k_{1}^{2} k_{2}-2 k_{1}^{2} N+k_{1} k_{2}^{2} N^{2}-3 k_{1} k_{2}^{2}+2 k_{1} k_{2} N^{3}-8 k_{1} k_{2} N\right.\right.} \\
& \left.\left.-4 k_{1} N^{2}-2 k_{2}^{2} N-4 k_{2} N^{2}-2 N^{3}\right)\right] /\left[2(N-2)(N+2)\left(N^{2}-3\right)\left(k_{1}+N\right)\left(k_{1}+k_{2}+N\right)\right. \\
& \left.\times D\left(k_{1}, k_{2}, N\right)\right], \\
c_{2}= & {\left[-2 N\left(N^{2}+1\right)\left(10 k_{1}^{3} k_{2} N^{2}-30 k_{1}^{3} k_{2}-20 k_{1}^{3} N+30 k_{1}^{2} k_{2}^{2} N^{2}-90 k_{1}^{2} k_{2}^{2}+35 k_{1}^{2} k_{2} N^{3}\right.\right.} \\
& -133 k_{1}^{2} k_{2} N-38 k_{1}^{2} N^{2}+20 k_{1} k_{2}^{3} N^{2}-60 k_{1} k_{2}^{3}+55 k_{1} k_{2}^{2} N^{3}-161 k_{1} k_{2}^{2} N+30 k_{1} k_{2} N^{4}
\end{aligned}
$$




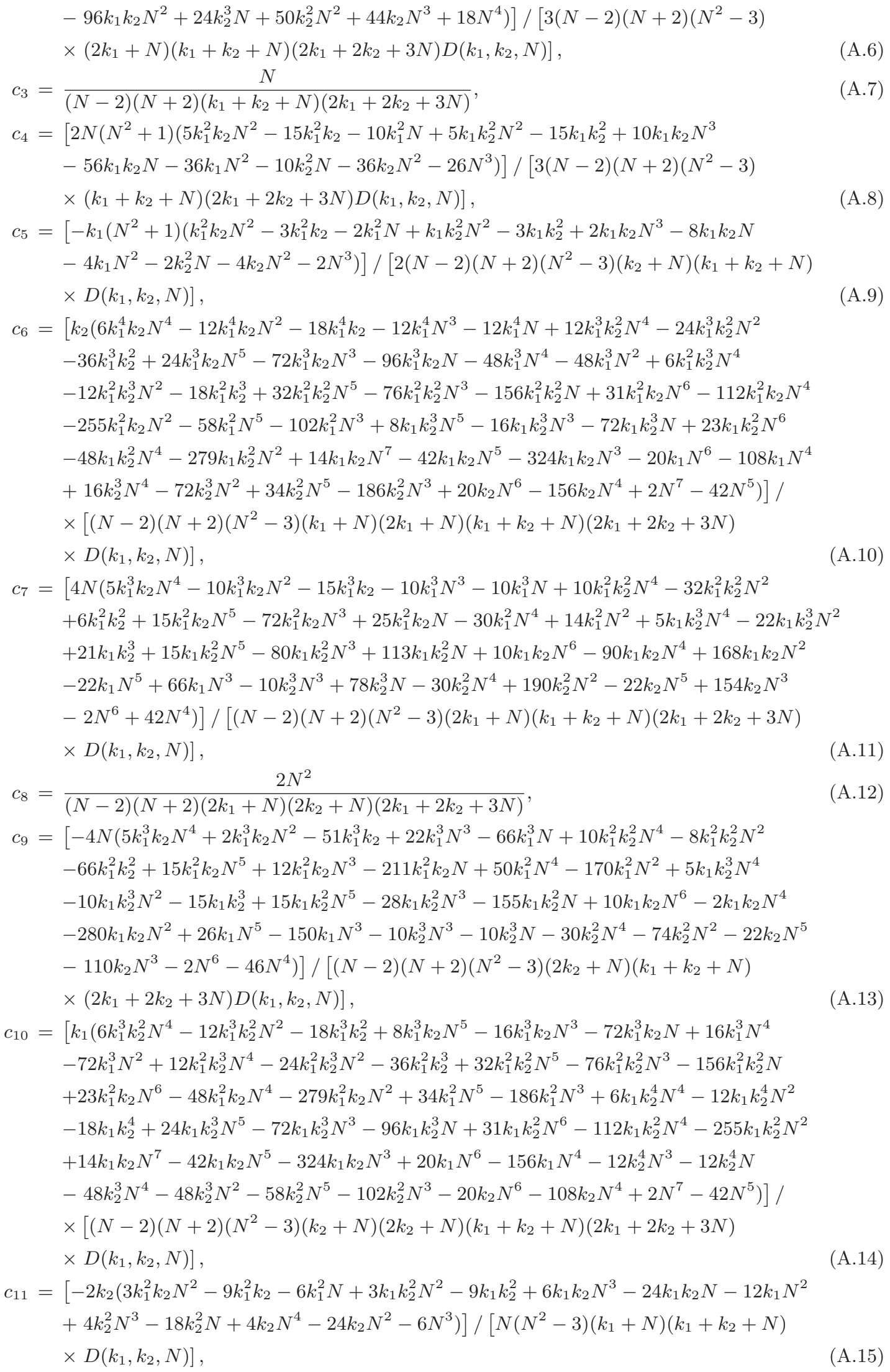




$$
\begin{aligned}
& c_{12}=\left[8 \left(-10 k_{1}^{3} k_{2} N^{2}+30 k_{1}^{3} k_{2}+20 k_{1}^{3} N-14 k_{1}^{2} k_{2}^{2} N^{2}+42 k_{1}^{2} k_{2}^{2}-19 k_{1}^{2} k_{2} N^{3}+85 k_{1}^{2} k_{2} N\right.\right. \\
& +38 k_{1}^{2} N^{2}-4 k_{1} k_{2}^{3} N^{2}+12 k_{1} k_{2}^{3}-7 k_{1} k_{2}^{2} N^{3}+17 k_{1} k_{2}^{2} N+2 k_{1} k_{2} N^{4}+8 k_{2}^{3} N^{3}-48 k_{2}^{3} N \\
& \left.\left.+20 k_{2}^{2} N^{4}-110 k_{2}^{2} N^{2}+12 k_{2} N^{5}-80 k_{2} N^{3}-18 N^{4}\right)\right] /\left[\left(N^{2}-3\right)\left(2 k_{1}+N\right)\right. \\
& \left.\times\left(k_{1}+k_{2}+N\right)\left(2 k_{1}+2 k_{2}+3 N\right) D\left(k_{1}, k_{2}, N\right)\right], \\
& c_{13}=\left[-4\left(3 k_{1}^{2} k_{2} N^{2}-9 k_{1}^{2} k_{2}-22 k_{1}^{2} N+3 k_{1} k_{2}^{2} N^{2}-9 k_{1} k_{2}^{2}+2 k_{1} k_{2} N^{3}-44 k_{1} k_{2} N-44 k_{1} N^{2}\right.\right. \\
& \left.\left.-22 k_{2}^{2} N-44 k_{2} N^{2}-22 N^{3}\right)\right] /\left[\left(N^{2}+1\right)\left(k_{1}+k_{2}+N\right)\left(2 k_{1}+2 k_{2}+3 N\right)\right. \\
& \left.\times D\left(k_{1}, k_{2}, N\right)\right] \text {, } \\
& c_{14}=\left[8 \left(8 k_{1}^{3} N^{2}-24 k_{1}^{3}+13 k_{1}^{2} k_{2} N^{2}-39 k_{1}^{2} k_{2}+20 k_{1}^{2} N^{3}-70 k_{1}^{2} N+5 k_{1} k_{2}^{2} N^{2}-15 k_{1} k_{2}^{2}\right.\right. \\
& \left.\left.+18 k_{1} k_{2} N^{3}-80 k_{1} k_{2} N+12 k_{1} N^{4}-72 k_{1} N^{2}-10 k_{2}^{2} N-36 k_{2} N^{2}-26 N^{3}\right)\right] /\left[\left(N^{2}-3\right)\right. \\
& \left.\times\left(k_{1}+k_{2}+N\right)\left(2 k_{1}+2 k_{2}+3 N\right) D\left(k_{1}, k_{2}, N\right)\right] \text {, } \\
& c_{15}=\left[-2 k_{1}\left(3 k_{1}^{2} k_{2} N^{2}-9 k_{1}^{2} k_{2}+4 k_{1}^{2} N^{3}-18 k_{1}^{2} N+3 k_{1} k_{2}^{2} N^{2}-9 k_{1} k_{2}^{2}+6 k_{1} k_{2} N^{3}-24 k_{1} k_{2} N\right.\right. \\
& \left.\left.+4 k_{1} N^{4}-24 k_{1} N^{2}-6 k_{2}^{2} N-12 k_{2} N^{2}-6 N^{3}\right)\right] /\left[N\left(N^{2}-3\right)\left(k_{2}+N\right)\left(k_{1}+k_{2}+N\right)\right. \\
& \left.\times D\left(k_{1}, k_{2}, N\right)\right] \text {, } \\
& c_{17}=\left[2 \left(6 k_{1}^{4} k_{2} N^{2}-18 k_{1}^{4} k_{2}-12 k_{1}^{4} N+12 k_{1}^{3} k_{2}^{2} N^{2}-36 k_{1}^{3} k_{2}^{2}+29 k_{1}^{3} k_{2} N^{3}-111 k_{1}^{3} k_{2} N\right.\right. \\
& -58 k_{1}^{3} N^{2}+6 k_{1}^{2} k_{2}^{3} N^{2}-18 k_{1}^{2} k_{2}^{3}+42 k_{1}^{2} k_{2}^{2} N^{3}-150 k_{1}^{2} k_{2}^{2} N+46 k_{1}^{2} k_{2} N^{4}-230 k_{1}^{2} k_{2} N^{2} \\
& -88 k_{1}^{2} N^{3}+13 k_{1} k_{2}^{3} N^{3}-51 k_{1} k_{2}^{3} N+38 k_{1} k_{2}^{2} N^{4}-166 k_{1} k_{2}^{2} N^{2}+24 k_{1} k_{2} N^{5}-156 k_{1} k_{2} N^{3} \\
& \left.\left.-42 k_{1} N^{4}+6 k_{2}^{3} N^{2}+4 k_{2}^{2} N^{3}-2 k_{2} N^{4}\right)\right] /\left[3\left(2 k_{1}+N\right)\left(k_{1}+k_{2}+N\right)\left(2 k_{1}+2 k_{2}+3 N\right)\right. \\
& \left.\times D\left(k_{1}, k_{2}, N\right)\right], \\
& c_{20}=\left[-2\left(3 k_{1}^{3} k_{2} N^{4}-6 k_{1}^{3} k_{2} N^{2}-9 k_{1}^{3} k_{2}-6 k_{1}^{3} N^{3}-6 k_{1}^{3} N+6 k_{1}^{2} k_{2}^{2} N^{4}-12 k_{1}^{2} k_{2}^{2} N^{2}-18 k_{1}^{2} k_{2}^{2}\right.\right. \\
& +8 k_{1}^{2} k_{2} N^{5}-34 k_{1}^{2} k_{2} N^{3}-18 k_{1}^{2} k_{2} N-32 k_{1}^{2} N^{4}+12 k_{1}^{2} N^{2}+3 k_{1} k_{2}^{3} N^{4}-6 k_{1} k_{2}^{3} N^{2}-9 k_{1} k_{2}^{3} \\
& +8 k_{1} k_{2}^{2} N^{5}-34 k_{1} k_{2}^{2} N^{3}-18 k_{1} k_{2}^{2} N+4 k_{1} k_{2} N^{6}-64 k_{1} k_{2} N^{4}+24 k_{1} k_{2} N^{2}-46 k_{1} N^{5} \\
& \left.\left.+42 k_{1} N^{3}-6 k_{2}^{3} N^{3}-6 k_{2}^{3} N-32 k_{2}^{2} N^{4}+12 k_{2}^{2} N^{2}-46 k_{2} N^{5}+42 k_{2} N^{3}-20 N^{6}+24 N^{4}\right)\right] / \\
& \times\left[\left(N^{2}+1\right)\left(k_{1}+k_{2}+N\right)\left(2 k_{1}+2 k_{2}+3 N\right) D\left(k_{1}, k_{2}, N\right)\right], \\
& c_{22}=\left[2 ( k _ { 1 } + k _ { 2 } + 2 N ) \left(3 k_{1}^{2} k_{2} N^{2}-9 k_{1}^{2} k_{2}-6 k_{1}^{2} N+3 k_{1} k_{2}^{2} N^{2}-9 k_{1} k_{2}^{2}+2 k_{1} k_{2} N^{3}-12 k_{1} k_{2} N\right.\right. \\
& \left.\left.-4 k_{1} N^{2}-6 k_{2}^{2} N-4 k_{2} N^{2}+2 N^{3}\right)\right] /\left[3\left(k_{1}+k_{2}+N\right)\left(2 k_{1}+2 k_{2}+3 N\right)\right. \\
& \left.\times D\left(k_{1}, k_{2}, N\right)\right], \\
& c_{23}=\left[-8\left(3 k_{1}^{2} k_{2} N^{2}-9 k_{1}^{2} k_{2}+8 k_{1}^{2} N^{3}-14 k_{1}^{2} N+3 k_{1} k_{2}^{2} N^{2}-9 k_{1} k_{2}^{2}+18 k_{1} k_{2} N^{3}-28 k_{1} k_{2} N\right.\right. \\
& \left.\left.+20 k_{1} N^{4}-24 k_{1} N^{2}+8 k_{2}^{2} N^{3}-14 k_{2}^{2} N+20 k_{2} N^{4}-24 k_{2} N^{2}+12 N^{5}-10 N^{3}\right)\right] / \\
& \times\left[\left(N^{2}+1\right)\left(k_{1}+k_{2}+N\right)\left(2 k_{1}+2 k_{2}+3 N\right) D\left(k_{1}, k_{2}, N\right)\right] \text {, }
\end{aligned}
$$

where

$$
\begin{aligned}
D\left(k_{1}, k_{2}, N\right) \equiv & 5 k_{1}^{2} k_{2} N^{2}+17 k_{1}^{2} k_{2}+22 k_{1}^{2} N+5 k_{1} k_{2}^{2} N^{2}+17 k_{1} k_{2}^{2}+10 k_{1} k_{2} N^{3}+56 k_{1} k_{2} N \\
& +44 k_{1} N^{2}+22 k_{2}^{2} N+44 k_{2} N^{2}+22 N^{3} .
\end{aligned}
$$

Note that $\left(c_{i}+b_{i}\right)$ in [7] is replaced with $c_{i}$ here. Of course, in the coset model (1.1), the levels are fixed to $k_{1}=1$ and $k_{2}=k$.

\section{B Intermediate spin-5 field contents in the second-order pole of the OPE $W^{(3)}(z) W^{(4)}(w)$}

The second-order pole in $W^{(3)}(z) W^{(4)}(w)(3.14)$ can be calculated explicitly using the spin-3 current in (3.7) and the spin-4 current in (3.9). Consider the first term $Q(z) \equiv$ $d^{a b c} J^{a} J^{b} J^{c}(z)$ of (3.7) and make an OPE between $Q(z)$ and 21 terms in the spin- 4 current. 
For the spin- 4 current, only the fields that did not have a $d$ symbol were considered. Twelve quartic terms can be obtained from the following five terms with the free indices.

$$
J^{a} J^{b} J^{c} J^{d}(w), \quad J^{a} J^{b} J^{c} K^{d}(w), \quad J^{a} J^{b} K^{c} K^{d}(w), \quad J^{a} K^{b} K^{c} K^{d}(w), \quad K^{a} K^{b} K^{c} K^{d}(w) .
$$

After calculating the OPE between $Q(z)$ with the five fields in (B.1), the nine OPEs between $Q(z)$ with the remaining nine terms in the spin- 4 currents are given. The following fourteen OPEs are then obtained:

$$
\begin{aligned}
Q(z) J^{a} J^{b} J^{c} J^{d}(w) \mid \frac{1}{(z-w)^{2}} & =-3\left(k_{1}+N\right)\left(Q^{a} J^{b} J^{c} J^{d}+J^{a} Q^{b} J^{c} J^{d}+J^{a} J^{b} Q^{c} J^{d}+J^{a} J^{b} J^{c} Q^{d}\right)(w), \\
Q(z) J^{a} J^{b} J^{c} K^{d}(w) \mid \frac{1}{(z-w)^{2}} & =-3\left(k_{1}+N\right)\left(Q^{a} J^{b} J^{c} K^{d}+J^{a} Q^{b} J^{c} K^{d}+J^{a} J^{b} Q^{c} K^{d}\right)(w), \\
Q(z) J^{a} J^{b} K^{c} K^{d}(w) \mid \frac{1}{(z-w)^{2}} & =-3\left(k_{1}+N\right)\left(Q^{a} J^{b} K^{c} K^{d}+J^{a} Q^{b} K^{c} K^{d}\right)(w), \\
Q(z) J^{a} K^{b} K^{c} K^{d}(w) \mid \frac{1}{(z-w)^{2}} & =-3\left(k_{1}+N\right) Q^{a} K^{b} K^{c} K^{d}(w), \\
Q(z) K^{a} K^{b} K^{c} K^{d}(w) \mid \frac{1}{(z-w)^{2}} & =0, \\
Q(z) \partial^{2} J^{a} K^{a}(w) \mid \frac{1}{(z-w)^{2}} & =-9\left(k_{1}+N\right) \partial^{2} Q^{a} K^{a}(w), \\
Q(z) \partial J^{a} \partial K^{a}(w) \mid \frac{1}{(z-w)^{2}} & =-6\left(k_{1}+N\right) \partial Q^{a} \partial K^{a}(w), \\
Q(z) J^{a} \partial^{2} K^{a}(w) \mid \frac{1}{(z-w)^{2}} & =-3\left(k_{1}+N\right) Q^{a} \partial^{2} K^{a}(w), \\
Q(z) f^{a b c} \partial J^{a} J^{b} K^{c}(w) \mid \frac{1}{(z-w)^{2}} & =-3\left(k_{1}+N\right)\left(2 f^{a b c} \partial Q^{a} J^{b} K^{c}+f^{a b c} \partial J^{a} Q^{b} K^{c}\right)(w), \\
Q(z) f^{a b c} \partial K^{a} K^{b} J^{c}(w) \mid \frac{1}{(z-w)^{2}} & =-3\left(k_{1}+N\right) \partial K^{a} K^{b} Q^{c}(w), \\
Q(z) \partial^{2} J^{a} J^{a}(w) \mid \frac{1}{(z-w)^{2}} & =-3\left(k_{1}+N\right)\left(3 J^{a} \partial^{2} Q^{a}+\partial^{2} J^{a} Q^{a}\right)(w), \\
Q(z) \partial^{2} K^{a} K^{a}(w) \mid \frac{1}{(z-w)^{2}} & =0 \\
Q(z) \partial J^{a} \partial J^{a}(w) \mid \frac{1}{(z-w)^{2}} & =-12\left(k_{1}+N\right) \partial J^{a} \partial Q^{a}(w), \\
Q(z) \partial K^{a} \partial K^{a}(w) \mid \frac{1}{(z-w)^{2}} & =0 .
\end{aligned}
$$

For the quartic terms in the left hand side, the extra $d$ or $\delta$ symbols are multiplied to obtain the final result for the second order pole. Further simplifications for the right hand side of (B.2) is needed to analyze the zero mode eigenvalue equations. In other words, field $Q^{a}(z)$ should be located at the right hand side of field $J^{a}(z)$ by moving $Q^{a}(z)$ to the right. Field $K^{a}(z)$ can be placed to the right. This issue will be addressed in appendix $C$.

Let us introduce spin-2 operator for convenience.

$$
L^{a}(z) \equiv d^{a b c} J^{b} K^{c}(z) .
$$

Consider the second term $d^{a b c} J^{a} J^{b} K^{c}(z) \equiv K^{e} Q^{e}(z)$ of (3.7) and calculate the fourteen OPEs as follows:

$$
\begin{aligned}
& \left.K^{e} Q^{e}(z) J^{a} J^{b} J^{c} J^{d}(w)\right|_{\frac{1}{(z-w)^{2}}}=-\left(2 k_{1}+N\right)\left[L^{a} J^{b} J^{c} J^{d}+J^{a} L^{b} J^{c} J^{d}+J^{a} J^{b} L^{c} J^{d}\right. \\
& +J^{a} J^{b} J^{c} L^{d}-f^{a f g} d^{b e g} \partial\left(J^{e} K^{f}\right) J^{c} J^{d}-f^{a f g} d^{c e g} J^{b} \partial\left(J^{e} K^{f}\right) J^{d}-f^{a f g} d^{d e g} J^{b} J^{c} \partial\left(J^{e} K^{f}\right) \\
& \left.-f^{b f g} d^{c e g} J^{a} \partial\left(J^{e} K^{f}\right) J^{d}-f^{b f g} d^{d e g} J^{a} J^{c} \partial\left(J^{e} K^{f}\right)-f^{c f g} d^{d e g} J^{a} J^{b} \partial\left(J^{e} K^{f}\right)\right](w) \\
& -f^{a f g} f^{b e g}\left(Q^{e} K^{f}\right) J^{c} J^{d}(w)-f^{a f g} f^{c e g} J^{b}\left(Q^{e} K^{f}\right) J^{d}(w)-f^{a f g} f^{d e g} J^{b} J^{c}\left(Q^{e} K^{f}\right)(w) \\
& -f^{b f g} f^{c e g} J^{a}\left(Q^{e} K^{f}\right) J^{d}(w)-f^{b f g} f^{d e g} J^{a} J^{c}\left(Q^{e} K^{f}\right)(w)-f^{c f g} f^{d e g} J^{a} J^{b}\left(Q^{e} K^{f}\right)(w),
\end{aligned}
$$


$\left.K^{e} Q^{e}(z) J^{a} J^{b} J^{c} K^{d}(w)\right|_{\frac{1}{(z-w)^{2}}}=-k_{2} Q^{d} J^{a} J^{b} J^{c}(w)-\left(2 k_{1}+N\right)\left[K^{d} L^{a} J^{b} J^{c}+K^{d} J^{a} L^{b} J^{c}\right.$ $+K^{d} J^{a} J^{b} L^{c}-f^{a f g} d^{b e g} K^{d} \partial\left(J^{e} K^{f}\right) J^{c}-f^{a f g} d^{c e g} K^{d} J^{b} \partial\left(J^{e} K^{f}\right)-f^{b f g} d^{c e g} K^{d} J^{a} \partial\left(J^{e} K^{f}\right)$

$\left.+f^{d f g} d^{a e g} \partial\left(J^{e} K^{f}\right) J^{b} J^{c}+f^{d f g} d^{b e g} J^{a} \partial\left(J^{e} K^{f}\right) J^{c}+f^{d f g} d^{c e g} J^{a} J^{b} \partial\left(J^{e} K^{f}\right)\right](w)$

$-f^{a f g} f^{b e g} K^{d}\left(Q^{e} K^{f}\right) J^{c}(w)-f^{a f g} f^{c e g} K^{d} J^{b}\left(Q^{e} K^{f}\right)(w)-f^{b f g} f^{c e g} K^{d} J^{a}\left(Q^{e} K^{f}\right)(w)$

$+f^{d f g} f^{a e g}\left(Q^{e} K^{f}\right) J^{b} J^{c}(w)+f^{d f g} f^{b e g} J^{a}\left(Q^{e} K^{f}\right) J^{c}(w)+f^{d f g} f^{c e g} J^{a} J^{b}\left(Q^{e} K^{f}\right)(w)$,

$\left.K^{e} Q^{e}(z) J^{a} J^{b} K^{c} K^{d}(w)\right|_{\frac{1}{(z-w)^{2}}}=-k_{2} J^{a} J^{b} Q^{c} K^{d}(w)-k_{2} J^{a} J^{b} K^{c} Q^{d}(w)-\left(2 k_{1}+N\right)\left[L^{a} J^{b} K^{c} K^{d}\right.$

$\left.+J^{a} L^{b} K^{c} K^{d}-f^{a f g} d^{b e g} \partial\left(J^{e} K^{f}\right) K^{c} K^{d}\right](w)+k_{2}\left(f^{a c e} J^{b} \partial Q^{e} K^{d}+f^{a d e} J^{b} K^{c} \partial Q^{e}\right.$

$\left.+f^{b c e} J^{a} \partial Q^{e} K^{d}+f^{b d e} J^{a} K^{c} \partial Q^{e}-f^{c d e} J^{a} J^{b} \partial Q^{e}\right)(w)-f^{a f g} f^{b e g}\left(Q^{e} K^{f}\right) K^{c} K^{d}(w)$

$+f^{c f g} f^{a e g} J^{b}\left(Q^{e} K^{f}\right) K^{d}(w)+f^{d f g} f^{a e g} J^{b} K^{c}\left(Q^{e} K^{f}\right)(w)+f^{b e g} f^{c f g} J^{a}\left(Q^{e} K^{f}\right) K^{d}(w)$

$+f^{b e g} f^{d f g} J^{a} K^{c}\left(Q^{e} K^{f}\right)(w)-f^{c e g} f^{d f g} J^{a} J^{b}\left(Q^{e} K^{f}\right)(w)$,

$\left.K^{e} Q^{e}(z) J^{a} K^{b} K^{c} K^{d}(w)\right|_{\frac{1}{(z-w)^{2}}}=-\left(2 k_{1}+N\right) L^{a} K^{b} K^{c} K^{d}(w)-k_{2}\left(J^{a} Q^{b} K^{c} K^{d}+J^{a} K^{b} Q^{c} K^{d}\right.$

$+J^{a} K^{b} K^{c} Q^{d}-f^{a b e} \partial Q^{e} K^{c} K^{d}-f^{a c e} K^{b} \partial Q^{e} K^{d}-f^{a d e} K^{b} K^{c} \partial Q^{e}+f^{b c e} J^{a} \partial Q^{e} K^{d}$

$\left.+f^{b d e} J^{a} K^{c} \partial Q^{e}+f^{c d e} J^{a} K^{b} \partial Q^{e}\right)(w)+f^{a e g} f^{b f g}\left(Q^{e} K^{f}\right) K^{c} K^{d}(w)+f^{a e g} f^{c f g} K^{b}\left(Q^{e} K^{f}\right) K^{d}(w)$

$+f^{a e g} f^{d f g} K^{b} K^{c}\left(Q^{e} K^{f}\right)(w)-f^{b e g} f^{c f g} J^{a}\left(Q^{e} K^{f}\right) K^{d}(w)-f^{b e g} f^{d f g} J^{a} K^{c}\left(Q^{e} K^{f}\right)(w)$

$-f^{c e g} f^{d f g} J^{a} K^{b}\left(Q^{e} K^{f}\right)(w)$,

$\left.K^{e} Q^{e}(z) K^{a} K^{b} K^{c} K^{d}(w)\right|_{\frac{1}{(z-w)^{2}}}=-k_{2}\left(Q^{a} K^{b} K^{c} K^{d}+K^{a} Q^{b} K^{c} K^{d}+K^{a} K^{b} Q^{c} K^{d}\right.$

$+K^{a} K^{b} K^{c} Q^{d}+f^{a b e} \partial Q^{e} K^{c} K^{d}+f^{a c e} K^{b} \partial Q^{e} K^{d}+f^{a d e} K^{b} K^{c} \partial Q^{e}+f^{b c e} K^{a} \partial Q^{e} K^{d}$

$\left.+f^{b d e} K^{a} K^{c} \partial Q^{e}+f^{c d e} K^{a} K^{b} \partial Q^{e}\right)(w)-f^{a e g} f^{b f g}\left(Q^{e} K^{f}\right) K^{c} K^{d}(w)-f^{a e g} f^{c f g} K^{b}\left(Q^{e} K^{f}\right) K^{d}(w)$

$-f^{a e g} f^{d f g} K^{b} K^{c}\left(Q^{e} K^{f}\right)(w)-f^{b e g} f^{c f g} K^{a}\left(Q^{e} K^{f}\right) K^{d}(w)-f^{b e g} f^{d f g} K^{a} K^{c}\left(Q^{e} K^{f}\right)(w)$

$-f^{c e g} f^{d f g} K^{a} K^{b}\left(Q^{e} K^{f}\right)(w)$,

$\left.K^{e} Q^{e}(z) \partial^{2} J^{a} K^{a}(w)\right|_{\frac{1}{(z-w)^{2}}}=-3\left(2 k_{1}+N\right) \partial^{2} L^{a} K^{a}(w)-k_{2} \partial^{2} J^{a} Q^{a}(w)+2 f^{a b c} \partial\left(Q^{a} K^{b}\right) K^{c}(w)$,

$\left.K^{e} Q^{e}(z) \partial J^{a} \partial K^{a}(w)\right|_{\frac{1}{(z-w)^{2}}}=-2\left(2 k_{1}+N\right) \partial L^{a} \partial K^{a}(w)-2 k_{2} \partial J^{a} \partial Q^{a}(w)+f^{a b c}\left(Q^{a} K^{b}\right) \partial K^{c}(w)$ $-f^{a b c} \partial J^{a}\left(Q^{b} K^{c}\right)(w)$

$\left.K^{e} Q^{e}(z) J^{a} \partial^{2} K^{a}(w)\right|_{\frac{1}{(z-w)^{2}}}=-\left(2 k_{1}+N\right) L^{a} \partial^{2} K^{a}(w)-3 k_{2} J^{a} \partial^{2} Q^{a}(w)+2 N \partial^{2}\left(Q^{a} K^{a}\right)(w)$

$-2 f^{a b c} J^{a} \partial\left(Q^{b} K^{c}\right)(w)$,

$K^{e} Q^{e}(z) f^{a b c} \partial J^{a} J^{b} K^{c}(w) \mid \frac{1}{(z-w)^{2}}=k_{2}\left(-f^{a b c} \partial J^{a} J^{b} Q^{c}+2 N \partial J^{a} \partial Q^{a}\right)(w)$

$+\left(2 k_{1}+N\right)\left(-2 d^{a b e} f^{c d e} \partial\left(J^{a} K^{b}\right) J^{c} K^{d}+f^{a d e} d^{b c e} \partial J^{a}\left(J^{b} K^{c}\right) K^{d}\right)(w)+f^{a b e} f^{c d e}\left(Q^{a} K^{b}\right) J^{c} K^{d}(w)$

$+N f^{a b c} \partial J^{a} Q^{b} K^{c}(w)$

$\left.K^{e} Q^{e}(z) f^{a b c} \partial K^{a} K^{b} J^{c}(w)\right|_{\frac{1}{(z-w)^{2}}}=k_{2}\left(2 f^{a b c} \partial Q^{a} J^{b} K^{c}+f^{a b c} \partial K^{a} J^{b} Q^{c}-2 N \partial K^{a} \partial Q^{a}\right)(w)$

$-\left(2 k_{1}+N\right) f^{a d e} d^{b c e} \partial K^{a}\left(J^{b} K^{c}\right) K^{d}(w)+f^{a b e} f^{c d e}\left(Q^{a} K^{b}\right) J^{c} K^{d}(w)+N f^{a b c} Q^{a} \partial K^{b} K^{c}(w)$,

$\left.K^{e} Q^{e}(z) \partial^{2} J^{a} J^{a}(w)\right|_{\frac{1}{(z-w)^{2}}}=-\left(2 k_{1}+N\right)\left(3 \partial^{2} L^{a} J^{a}+\partial^{2} J^{a} L^{a}\right)(w)+2 f^{a b c} \partial\left(Q^{a} K^{b}\right) J^{c}(w)$,

$\left.K^{e} Q^{e}(z) \partial^{2} K^{a} K^{a}(w)\right|_{\frac{1}{(z-w)^{2}}}=-k_{2}\left(3 \partial^{2} Q^{a} K^{a}+Q^{a} \partial^{2} K^{a}\right)(w)-2 f^{a b c} \partial\left(Q^{a} K^{b}\right) K^{c}(w)$,

$\left.K^{e} Q^{e}(z) \partial J^{a} \partial J^{a}(w)\right|_{\frac{1}{(z-w)^{2}}}=-2\left(2 k_{1}+N\right)\left(\partial L^{a} \partial J^{a}+\partial J^{a} \partial L^{a}\right)(w)+f^{a b c}\left(\left(Q^{a} K^{b}\right) \partial J^{c}\right.$

$\left.+\partial J^{a}\left(Q^{b} K^{c}\right)\right)(w)$

$\left.K^{e} Q^{e}(z) \partial K^{a} \partial K^{a}(w)\right|_{\frac{1}{(z-w)^{2}}}=-4 k_{2} \partial Q^{a} \partial K^{a}(w)-f^{a b c}\left(\left(Q^{a} K^{b}\right) \partial K^{c}+\partial K^{a}\left(Q^{b} K^{c}\right)\right)(w) . \quad$ (B. 4$)$

Further simplifications for the right hand side of (B.4) with the appropriate normal ordering procedure are needed. 
The second order pole for the third term $d^{a b c} J^{a} K^{b} K^{c}(z) \equiv J^{e} R^{e}(z)$ of (3.7) and the spin-4 current without using an explicit calculation can be obtained from the previous result (B.4)

$$
\begin{aligned}
& \left.J^{e} R^{e}(z) J^{a} J^{b} J^{c} J^{d}(w)\right|_{\frac{1}{(z-w)^{2}}}=\left.\left(\left.K^{e} Q^{e}(z) K^{a} K^{b} K^{c} K^{d}(w)\right|_{\frac{1}{(z-w)^{2}}}\right)\right|_{k_{1} \leftrightarrow k_{2}, J^{a} \leftrightarrow K^{a}, Q^{a} \leftrightarrow R^{a}}, \\
& \left.J^{e} R^{e}(z) J^{a} J^{b} J^{c} K^{d}(w)\right|_{\frac{1}{(z-w)^{2}}}=\left.\left(\left.K^{e} Q^{e}(z) J^{d} K^{a} K^{b} K^{c}(w)\right|_{\frac{1}{(z-w)^{2}}}\right)\right|_{k_{1} \leftrightarrow k_{2}, J^{a} \leftrightarrow K^{a}, Q^{a} \leftrightarrow R^{a}}, \\
& \left.J^{e} R^{e}(z) J^{a} J^{b} K^{c} K^{d}(w)\right|_{\frac{1}{(z-w)^{2}}}=\left.\left(\left.K^{e} Q^{e}(z) J^{c} J^{d} K^{a} K^{b}(w)\right|_{\frac{1}{(z-w)^{2}}}\right)\right|_{k_{1} \leftrightarrow k_{2}, J^{a} \leftrightarrow K^{a}, Q^{a} \leftrightarrow R^{a}}, \\
& \left.J^{e} R^{e}(z) J^{a} K^{b} K^{c} K^{d}(w)\right|_{\frac{1}{(z-w)^{2}}}=\left.\left(\left.K^{e} Q^{e}(z) J^{b} J^{c} J^{d} K^{a}(w)\right|_{\frac{1}{(z-w)^{2}}}\right)\right|_{k_{1} \leftrightarrow k_{2}, J^{a} \leftrightarrow K^{a}, Q^{a} \leftrightarrow R^{a}}, \\
& \left.J^{e} R^{e}(z) K^{a} K^{b} K^{c} K^{d}(w)\right|_{\frac{1}{(z-w)^{2}}}=\left.\left(\left.K^{e} Q^{e}(z) J^{a} J^{b} J^{c} J^{d}(w)\right|_{\frac{1}{(z-w)^{2}}}\right)\right|_{k_{1} \leftrightarrow k_{2}, J^{a} \leftrightarrow K^{a}, Q^{a} \leftrightarrow R^{a}}, \\
& \left.J^{e} R^{e}(z) \partial^{2} J^{a} K^{a}(w)\right|_{\frac{1}{(z-w)^{2}}}=\left.\left(\left.K^{e} Q^{e}(z) J^{a} \partial^{2} K^{a}(w)\right|_{\frac{1}{(z-w)^{2}}}\right)\right|_{k_{1} \leftrightarrow k_{2}, J^{a} \leftrightarrow K^{a}, Q^{a} \leftrightarrow R^{a}}, \\
& \left.J^{e} R^{e}(z) \partial J^{a} \partial K^{a}(w)\right|_{\frac{1}{(z-w)^{2}}}=\left.\left(\left.K^{e} Q^{e}(z) \partial J^{a} \partial K^{a}(w)\right|_{\frac{1}{(z-w)^{2}}}\right)\right|_{k_{1} \leftrightarrow k_{2}, J^{a} \leftrightarrow K^{a}, Q^{a} \leftrightarrow R^{a}}, \\
& J^{e} R^{e}(z) J^{a} \partial^{2} K^{a}(w)\left|\frac{1}{(z-w)^{2}}=\left(K^{e} Q^{e}(z) \partial^{2} J^{a} K^{a}(w) \mid \frac{1}{(z-w)^{2}}\right)\right|_{k_{1} \leftrightarrow k_{2}, J^{a} \leftrightarrow K^{a}, Q^{a} \leftrightarrow R^{a}}, \\
& \left.J^{e} R^{e}(z) f^{a b c} \partial J^{a} J^{b} K^{c}(w)\right|_{\frac{1}{(z-w)^{2}}}=\left.\left(\left.K^{e} Q^{e}(z) f^{a b c} \partial K^{a} K^{b} J^{c}(w)\right|_{\frac{1}{(z-w)^{2}}}\right)\right|_{k_{1} \leftrightarrow k_{2}, J^{a} \leftrightarrow K^{a}, Q^{a} \leftrightarrow R^{a}}, \\
& \left.J^{e} R^{e}(z) f^{a b c} \partial K^{a} K^{b} J^{c}(w)\right|_{\frac{1}{(z-w)^{2}}}=\left(\left.K^{e} Q^{e}(z) f^{a b c} \partial J^{a} J^{b} K^{c}(w)\right|_{\frac{1}{(z-w)^{2}}}\right) \mid k_{1} \leftrightarrow k_{2}, J^{a} \leftrightarrow K^{a}, Q^{a} \leftrightarrow R^{a}, \\
& \left.J^{e} R^{e}(z) \partial^{2} J^{a} J^{a}(w)\right|_{\frac{1}{(z-w)^{2}}}=\left.\left(\left.K^{e} Q^{e}(z) \partial^{2} K^{a} K^{a}(w)\right|_{\frac{1}{(z-w)^{2}}}\right)\right|_{k_{1} \leftrightarrow k_{2}, J^{a} \leftrightarrow K^{a}, Q^{a} \leftrightarrow R^{a}}, \\
& \left.J^{e} R^{e}(z) \partial^{2} K^{a} K^{a}(w)\right|_{\frac{1}{(z-w)^{2}}}=\left.\left(\left.K^{e} Q^{e}(z) \partial^{2} J^{a} J^{a}(w)\right|_{\frac{1}{(z-w)^{2}}}\right)\right|_{k_{1} \leftrightarrow k_{2}, J^{a} \leftrightarrow K^{a}, Q^{a} \leftrightarrow R^{a}}, \\
& \left.J^{e} R^{e}(z) \partial J^{a} \partial J^{a}(w)\right|_{\frac{1}{(z-w)^{2}}}=\left.\left(\left.K^{e} Q^{e}(z) \partial K^{a} \partial K^{a}(w)\right|_{\frac{1}{(z-w)^{2}}}\right)\right|_{k_{1} \leftrightarrow k_{2}, J^{a} \leftrightarrow K^{a}, Q^{a} \leftrightarrow R^{a}}, \\
& \left.J^{e} R^{e}(z) \partial K^{a} \partial K^{a}(w)\right|_{\frac{1}{(z-w)^{2}}}=\left.\left(\left.K^{e} Q^{e}(z) \partial J^{a} \partial J^{a}(w)\right|_{\frac{1}{(z-w)^{2}}}\right)\right|_{k_{1} \leftrightarrow k_{2}, J^{a} \leftrightarrow K^{a}, Q^{a} \leftrightarrow R^{a}} .
\end{aligned}
$$

Finally, the second order pole in the OPE between the fourth term $d^{a b c} K^{a} K^{b} K^{c}(z) \equiv$ $R(z)$ of (3.7) and the spin-4 current can be obtained from the previous result (B.2).

$$
\begin{aligned}
& \left.R(z) J^{a} J^{b} J^{c} J^{d}(w)\right|_{\frac{1}{(z-w)^{2}}}=0, \\
& \left.R(z) J^{a} J^{b} J^{c} K^{d}(w)\right|_{\frac{1}{(z-w)^{2}}}=\left.\left(\left.Q(z) J^{d} K^{a} K^{b} K^{c}(w)\right|_{\frac{1}{(z-w)^{2}}}\right)\right|_{k_{1} \leftrightarrow k_{2}, J^{a} \leftrightarrow K^{a}, Q^{a} \leftrightarrow R^{a}}, \\
& \left.R(z) J^{a} J^{b} K^{c} K^{d}(w)\right|_{\frac{1}{(z-w)^{2}}}=\left.\left(\left.Q(z) J^{c} J^{d} K^{a} K^{b}(w)\right|_{\frac{1}{(z-w)^{2}}}\right)\right|_{k_{1} \leftrightarrow k_{2}, J^{a} \leftrightarrow K^{a}, Q^{a} \leftrightarrow R^{a}}, \\
& \left.R(z) J^{a} K^{b} K^{c} K^{d}(w)\right|_{\frac{1}{(z-w)^{2}}}=\left.\left(\left.Q(z) J^{b} J^{c} J^{d} K^{a}(w)\right|_{\frac{1}{(z-w)^{2}}}\right)\right|_{k_{1} \leftrightarrow k_{2}, J^{a} \leftrightarrow K^{a}, Q^{a} \leftrightarrow R^{a}}, \\
& \left.R(z) K^{a} K^{b} K^{c} K^{d}(w)\right|_{\frac{1}{(z-w)^{2}}}=\left(\left.Q(z) J^{a} J^{b} J^{c} J^{d}(w)\right|_{\frac{1}{(z-w)^{2}}}\right) \mid k_{1} \leftrightarrow k_{2}, J^{a} \leftrightarrow K^{a}, Q^{a} \leftrightarrow R^{a}, \\
& \left.R(z) \partial^{2} J^{a} K^{a}(w)\right|_{\frac{1}{(z-w)^{2}}}=\left.\left(\left.Q(z) J^{a} \partial^{2} K^{a}(w)\right|_{\frac{1}{(z-w)^{2}}}\right)\right|_{k_{1} \leftrightarrow k_{2}, J^{a} \leftrightarrow K^{a}, Q^{a} \leftrightarrow R^{a}}, \\
& \left.R(z) \partial J^{a} \partial K^{a}(w)\right|_{\frac{1}{(z-w)^{2}}}=\left(\left.Q(z) \partial J^{a} \partial K^{a}(w)\right|_{\frac{1}{(z-w)^{2}}}\right) \mid k_{1} \leftrightarrow k_{2}, J^{a} \leftrightarrow K^{a}, Q^{a} \leftrightarrow R^{a}, \\
& \left.R(z) J^{a} \partial^{2} K^{a}(w)\right|_{\frac{1}{(z-w)^{2}}}=\left.\left(\left.Q(z) \partial^{2} J^{a} K^{a}(w)\right|_{\frac{1}{(z-w)^{2}}}\right)\right|_{k_{1} \leftrightarrow k_{2}, J^{a} \leftrightarrow K^{a}, Q^{a} \leftrightarrow R^{a}},
\end{aligned}
$$




$$
\begin{aligned}
\left.R(z) f^{a b c} \partial J^{a} J^{b} K^{c}(w)\right|_{\frac{1}{(z-w)^{2}}} & =\left.\left(\left.Q(z) f^{a b c} \partial K^{a} K^{b} J^{c}(w)\right|_{\frac{1}{(z-w)^{2}}}\right)\right|_{k_{1} \leftrightarrow k_{2}, J^{a} \leftrightarrow K^{a}, Q^{a} \leftrightarrow R^{a},}, \\
\left.R(z) f^{a b c} \partial K^{a} K^{b} J^{c}(w)\right|_{\frac{1}{(z-w)^{2}}} & =\left.\left(\left.Q(z) f^{a b c} \partial J^{a} J^{b} K^{c}(w)\right|_{\frac{1}{(z-w)^{2}}}\right)\right|_{k_{1} \leftrightarrow k_{2}, J^{a} \leftrightarrow K^{a}, Q^{a} \leftrightarrow R^{a},} \\
\left.R(z) \partial^{2} J^{a} J^{a}(w)\right|_{\frac{1}{(z-w)^{2}}} & =0 \\
\left.R(z) \partial^{2} K^{a} K^{a}(w)\right|_{\frac{1}{(z-w)^{2}}} & =\left.\left(\left.Q(z) \partial^{2} J^{a} J^{a}(w)\right|_{\frac{1}{(z-w)^{2}}}\right)\right|_{k_{1} \leftrightarrow k_{2}, J^{a} \leftrightarrow K^{a}, Q^{a} \leftrightarrow R^{a}}, \\
\left.R(z) \partial J^{a} \partial J^{a}(w)\right|_{\frac{1}{(z-w)^{2}}} & =0 \\
\left.R(z) \partial K^{a} \partial K^{a}(w)\right|_{\frac{1}{(z-w)^{2}}} & =\left.\left(\left.Q(z) \partial J^{a} \partial J^{a}(w)\right|_{\frac{1}{(z-w)^{2}}}\right)\right|_{k_{1} \leftrightarrow k_{2}, J^{a} \leftrightarrow K^{a}, Q^{a} \leftrightarrow R^{a}} .
\end{aligned}
$$

Therefore, the second order pole structures are exhausted. Further simplifications are given in appendix $C$.

\section{Details of the fully normal ordered spin-5 operator}

Thus far, the results from the previous appendices are not fully ordered in the sense of [34]. Further simplifications are needed with the help of the rearrangement lemma.

The following commutators with the appendices in [34] from OPEs (2.2) and (3.3).

$$
\begin{aligned}
{\left[J^{a}, J^{b}\right](z) } & =f^{a b c} \partial J^{c}(z), \quad\left[J^{a}, \partial J^{b}\right](z)=\frac{1}{2} f^{a b c} \partial^{2} J^{c}(z), \\
{\left[J^{a}, Q^{b}\right](z) } & =f^{a b c} \partial Q^{c}(z)+\frac{1}{2}\left(2 k_{1}+N\right) d^{a b c} \partial^{2} J^{c}(z), \\
{\left[J^{a}, \partial Q^{b}\right](z) } & =\frac{1}{2} f^{a b c} \partial^{2} Q^{c}(z)+\frac{1}{6}\left(2 k_{1}+N\right) d^{a b c} \partial^{3} J^{c}(z) .
\end{aligned}
$$

For example, take $\left.Q(z) d^{a b e} d^{c d e} J^{a} J^{b} K^{c} K^{d}(w)\right|_{\frac{1}{(z-w)^{2}}}$ from the third equation of (B.2) in appendix $B$ by multiplying two $d$ symbols

$$
\left.Q(z) d^{a b e} d^{c d e} J^{a} J^{b} K^{c} K^{d}(w)\right|_{\frac{1}{(z-w)^{2}}}=-3\left(k_{1}+N\right) d^{a b e} d^{c d e}\left(Q^{a} J^{b} K^{c} K^{d}+J^{a} Q^{b} K^{c} K^{d}\right)(w) .
$$

As stated before, the field $Q^{a}(z)$ in (C.2) should be moved to the right. The right hand side of (C.2) can be expressed as

$$
-3\left(k_{1}+N\right) d^{a b e} d^{c d e}\left(J^{b} Q^{a} K^{c} K^{d}-\left[J^{b}, Q^{a}\right] K^{c} K^{d}+J^{a} Q^{b} K^{c} K^{d}\right)(w)
$$

and using the third equation of (C.1) this (C.3) can be expressed as

$$
-3\left(k_{1}+N\right) d^{a b e} d^{c d e}\left(-\left(f^{b a f} \partial Q^{f}+\frac{1}{2}\left(2 k_{1}+N\right) d^{b a f} \partial^{2} J^{f}\right) K^{c} K^{d}+2 J^{a} Q^{b} K^{c} K^{d}\right)(w) .
$$

Owing to the antisymmetric property of the $f$ symbol and the symmetric property of the $d$ symbol, the first term of (C.4) vanishes and the remaining terms are

$$
\frac{3}{2}\left(k_{1}+N\right)\left(2 k_{1}+N\right) d^{a b e} d^{a b f} d^{c d e} \partial^{2} J^{f} K^{c} K^{d}(w)-6\left(k_{1}+N\right) d^{a b e} d^{c d e} J^{a} Q^{b} K^{c} K^{d}(w) .
$$


The first term in (C.5) can be simplified using the identity between two $d$ symbols

$$
\frac{3}{N}\left(k_{1}+N\right)\left(2 k_{1}+N\right)\left(N^{2}-4\right) d^{a b c} \partial^{2} J^{a} K^{b} K^{c}(w)-6\left(k_{1}+N\right) d^{a b e} d^{c d e} J^{a} Q^{b} K^{c} K^{d}(w) .
$$

The last term of (C.6) can be simplified further as follows:

$$
\begin{aligned}
& d^{a b e} d^{c d e} J^{a} Q^{b} K^{c} K^{d}(z)=d^{a b e} d^{c d e} d^{b f g} J^{a}\left(J^{f} J^{g}\right) K^{c} K^{d}(z) \\
& \quad=d^{a b e} d^{c d e} d^{b f g}\left(J^{f} J^{g} J^{a} K^{c} K^{d}-\left[J^{f}, J^{a}\right] J^{g} K^{c} K^{d}-J^{f}\left[J^{g}, J^{a}\right] K^{c} K^{d}\right)(z)
\end{aligned}
$$

where the definition of $Q^{b}(z)$ is used. The last two terms of (C.7) can be expressed as

$$
-d^{a b e} d^{c d e} d^{b f g} f^{f a h} \partial J^{h} J^{g} K^{c} K^{d}(z)-d^{a b e} d^{c d e} d^{b f g} f^{g a h} J^{f} \partial J^{h} K^{c} K^{d}(z)
$$

after substituting the commutators in (C.1). Using the identity of $(A .7)$ of [7] (the triple product $d d f$ is reduced to a single $f$ ), this expression (C.8) can be simplified as

$$
-\frac{N^{2}-4}{N} f^{a b e} d^{c d e} \partial J^{a} J^{b} K^{c} K^{d}(z)+\frac{N^{2}-4}{N} f^{a b e} d^{c d e} J^{a} \partial J^{b} K^{c} K^{d}(z) .
$$

The field $\partial J^{a}$ in the first term of (C.9) should be moved to the right to yield the following:

$$
-\frac{N^{2}-4}{N} f^{a b e} d^{c d e}\left(J^{b} \partial J^{a} K^{c} K^{d}-\left[J^{b}, \partial J^{a}\right] K^{c} K^{d}\right)(z)+\frac{N^{2}-4}{N} f^{a b e} d^{c d e} J^{a} \partial J^{b} K^{c} K^{d}(z) .
$$

Again, the commutator can be simplified, and after a small calculation, (C.10) leads to

$$
\frac{N^{2}-4}{2 N} f^{a b e} d^{c d e} f^{b a f} \partial^{2} J^{f} K^{c} K^{d}(z)+\frac{2\left(N^{2}-4\right)}{N} f^{a b e} d^{c d e} J^{a} \partial J^{b} K^{c} K^{d}(z)
$$

The $f f$ term in (C.11) can be simplified further and the final fully normal ordered product becomes

$$
\begin{aligned}
d^{a b e} d^{c d e} J^{a} Q^{b} K^{c} K^{d}(z)= & d^{a b f} d^{f c g} d^{g d e} J^{a} J^{b} J^{c} K^{d} K^{e}(z)+\left(4-N^{2}\right) d^{a b c} \partial^{2} J^{a} K^{b} K^{c}(z) \\
& +\frac{2\left(N^{2}-4\right)}{N} f^{a b e} d^{c d e} J^{a} \partial J^{b} K^{c} K^{d}(z) .
\end{aligned}
$$

where the right hand side of (C.12) appears in the fourth, 47th, and 35th terms in (3.15). The final form for $\left.Q(z) d^{a b e} d^{c d e} J^{a} J^{b} K^{c} K^{d}(w)\right|_{\frac{1}{(z-w)^{2}}}$ can be obtained from (C.6) and (C.12) as follows:

$$
\begin{aligned}
& Q(z) d^{a b e} d^{c d e} J^{a} J^{b} K^{c} K^{d}(w) \mid \frac{1}{(z-w)^{2}} \\
& =-6\left(k_{1}+N\right) d^{a b f} d^{f c g} d^{g d e} J^{a} J^{b} J^{c} K^{d} K^{e}(w)-\frac{12}{N}\left(k_{1}+N\right)\left(N^{2}-4\right) f^{a b e} d^{c d e} J^{a} \partial J^{b} K^{c} K^{d}(w) \\
& +\frac{3}{N}\left(k_{1}+N\right)\left(N^{2}-4\right)\left(2 k_{1}+3 N\right) d^{a b c} \partial^{2} J^{a} K^{b} K^{c}(w) .
\end{aligned}
$$

This identity (C.13) can be used continually. 
The other commutators are needed to rearrange all the normal ordered products in (B.2), (B.4), (B.5) and (B.6), which are not fully normal ordered product. First, calculate the $\mathrm{OPE} J^{a}(z) J^{b} K^{c}(w)$. Recall the mixed spin-2 field (B.3).

$$
J^{a}(z) J^{b} K^{c}(w)=-\frac{1}{(z-w)^{2}} k_{1} \delta^{a b} K^{c}(w)+\frac{1}{(z-w)} f^{a b d} J^{d} K^{c}(w)+\cdots
$$

From the OPE (C.14), the following various commutators, via (A.10) of [34], are obtained

$$
\begin{aligned}
{\left[J^{a}, L^{b}\right](z) } & =\frac{1}{2} k_{1} d^{a b c} \partial^{2} K^{c}(z)+f^{a c d} d^{b c e} \partial\left(J^{d} K^{e}\right)(z), \\
{\left[J^{a}, \partial\left(J^{b} K^{c}\right)\right](z) } & =\frac{1}{6} k_{1} \delta^{a b} \partial^{3} K^{c}(z)+\frac{1}{2} f^{a b d} \partial^{2}\left(J^{d} K^{c}\right)(z), \\
{\left[J^{a}, \partial^{2}\left(J^{b} K^{c}\right)\right](z) } & =\frac{1}{12} k_{1} \delta^{a b} \partial^{4} K^{c}(z)+\frac{1}{3} f^{a b d} \partial^{3}\left(J^{d} K^{c}\right)(z), \\
{\left[K^{a}, L^{b}\right](z) } & =\left.\left(\left[J^{a}, L^{b}\right](z)\right)\right|_{k_{1} \leftrightarrow k_{2}, J^{a} \leftrightarrow K^{a},}, \\
{\left[K^{a}, \partial\left(J^{b} K^{c}\right)\right](z) } & =\left.\left(\left[J^{a}, \partial\left(J^{c} K^{b}\right)\right](z)\right)\right|_{k_{1} \leftrightarrow k_{2}, J^{a} \leftrightarrow K^{a}}, \\
{\left[K^{a}, \partial^{2}\left(J^{b} K^{c}\right)\right](z) } & =\left.\left(\left[J^{a}, \partial^{2}\left(J^{c} K^{b}\right)\right](z)\right)\right|_{k_{1} \leftrightarrow k_{2}, J^{a} \leftrightarrow K^{a} .}
\end{aligned}
$$

Similarly, the OPEs $J^{a}(z) Q^{b} K^{c}(w)$ and $K^{a}(z) Q^{b} K^{c}(w)$ can be derived

$$
\begin{aligned}
& J^{a}(z) Q^{b} K^{c}(w)=-\frac{1}{(z-w)^{2}}\left(2 k_{1}+N\right) d^{a b d} J^{d} K^{c}(w)+\frac{1}{(z-w)} f^{a b d} Q^{d} K^{c}(w)+\cdots \\
& K^{a}(z) Q^{b} K^{c}(w)=-\frac{1}{(z-w)^{2}} k_{2} \delta^{a c} Q^{b}(w)+\frac{1}{(z-w)} f^{a c d} Q^{b} K^{d}(w)+\cdots
\end{aligned}
$$

The relevant commutators, which can be obtained from (C.16), are as follows:

$$
\begin{aligned}
{\left[J^{a}, Q^{b} K^{c}\right](z) } & =\frac{1}{2}\left(2 k_{1}+N\right) d^{a b d} \partial^{2}\left(J^{d} K^{c}\right)(z)+f^{a b d} \partial\left(Q^{d} K^{c}\right)(z), \\
{\left[J^{a}, \partial\left(Q^{b} K^{c}\right)\right](z) } & =\frac{1}{6}\left(2 k_{1}+N\right) d^{a b d} \partial^{3}\left(J^{d} K^{c}\right)(z)+\frac{1}{2} f^{a b d} \partial^{2}\left(Q^{d} K^{c}\right)(z), \\
{\left[K^{a}, Q^{b} K^{c}\right](z) } & =\frac{1}{2} k_{2} \delta^{a c} \partial^{2} Q^{b}(z)+f^{a c d} \partial\left(Q^{b} K^{d}\right)(z), \\
{\left[K^{a}, \partial\left(Q^{b} K^{c}\right)\right](z) } & =\frac{1}{6} k_{2} \delta^{a c} \partial^{3} Q^{b}(z)+\frac{1}{2} f^{a c d} \partial^{2}\left(Q^{b} K^{d}\right)(z), \\
{\left[J^{a}, R^{b} J^{c}\right](z) } & =\left.\left(\left[K^{a}, Q^{b} K^{c}\right](z)\right)\right|_{k_{1} \leftrightarrow k_{2}, J^{a} \leftrightarrow K^{a}, Q^{a} \leftrightarrow R^{a}}, \\
{\left[J^{a}, \partial\left(R^{b} J^{c}\right)\right](z) } & =\left.\left(\left[K^{a}, \partial\left(Q^{b} K^{c}\right)\right](z)\right)\right|_{k_{1} \leftrightarrow k_{2}, J^{a} \leftrightarrow K^{a}, Q^{a} \leftrightarrow R^{a},}, \\
{\left[K^{a}, R^{b} J^{c}\right](z) } & =\left.\left(\left[J^{a}, Q^{b} K^{c}\right](z)\right)\right|_{k_{1} \leftrightarrow k_{2}, J^{a} \leftrightarrow K^{a}, Q^{a} \leftrightarrow R^{a},}, \\
{\left[K^{a}, \partial\left(R^{b} J^{c}\right)\right](z) } & =\left.\left(\left[J^{a}, \partial\left(Q^{b} K^{c}\right)\right](z)\right)\right|_{k_{1} \leftrightarrow k_{2}, J^{a} \leftrightarrow K^{a}, Q^{a} \leftrightarrow R^{a} .}
\end{aligned}
$$

Finally, the commutators $\left[J^{a}, T\right](z)$ and $\left[K^{a}, T\right](z)$ to rearrange the normal ordered product $T W^{(3)}(w)$ in (3.14) are needed. The OPE $J^{a}(z) T(w)$ are calculated as follows:

$$
\begin{aligned}
J^{a}(z) T(w)= & \frac{1}{(z-w)^{2}} \frac{1}{\left(k_{1}+k_{2}+N\right)}\left(k_{2} J^{a}-k_{1} K^{a}\right)(w) \\
& -\frac{1}{(z-w)} \frac{1}{\left(k_{1}+k_{2}+N\right)} f^{a b c} J^{b} K^{c}(w)+\cdots .
\end{aligned}
$$


The following commutators can be obtained from (C.18)

$$
\begin{aligned}
{\left[J^{a}, T\right](z) } & =-\frac{1}{2\left(k_{1}+k_{2}+N\right)}\left(k_{2} \partial^{2} J^{a}-k_{1} \partial^{2} K^{a}\right)(z)-\frac{1}{\left(k_{1}+k_{2}+N\right)} f^{a b c} \partial\left(J^{b} K^{c}\right)(z), \\
{\left[K^{a}, T\right](z) } & =\left.\left(\left[J^{a}, T\right](z)\right)\right|_{k_{1} \leftrightarrow k_{2}, J^{a} \leftrightarrow K^{a}} .
\end{aligned}
$$

All the commutators in (C.15), (C.17) and (C.19) are used to simplify the normal ordered product in appendix $B$.

Although this paper did not present all the fully normal ordered products leading to the 52 terms for the spin-5 current (3.15) (due to the space limit of this paper), the complicated calculations from appendices $B$ and $C$ to the final 52 terms can be performed.

\section{Explicit 52 coefficient functions of spin-5 Casimir operator}

The 52 relative coefficient functions appearing in the spin- 5 current are given by

$$
\begin{aligned}
& a_{1}=-\frac{6 k N(k+N)(2 k+N)^{2}}{(N-2)(N+2)^{2}(2 k+3 N+2)}, \\
& a_{2}=\left[6 ( k + N ) ( 2 k + N ) \left(30 k^{3} N^{4}+204 k^{3} N^{3}+382 k^{3} N^{2}+184 k^{3} N-120 k^{3}+65 k^{2} N^{5}\right.\right. \\
& +518 k^{2} N^{4}+1217 k^{2} N^{3}+960 k^{2} N^{2}-188 k^{2} N-240 k^{2}+10 k N^{6}+329 k N^{5}+1114 k N^{4} \\
& +1265 k N^{3}+90 k N^{2}-452 k N-120 k+70 N^{6}+264 N^{5}+374 N^{4}+108 N^{3}-152 N^{2} \\
& -80 N)] /\left[(N-2)(N+1)(N+2)^{2}(2 k+3 N+2) d(N, k)\right], \\
& a_{3}=\left[6 ( k + N ) ( 2 k + N ) \left(20 k^{3} N^{4}+66 k^{3} N^{3}+8 k^{3} N^{2}-14 k^{3} N+120 k^{3}+60 k^{2} N^{5}\right.\right. \\
& +207 k^{2} N^{4}+28 k^{2} N^{3}-145 k^{2} N^{2}+358 k^{2} N+240 k^{2}+40 k N^{6}+186 k N^{5}+71 k N^{4} \\
& -240 k N^{3}+215 k N^{2}+452 k N+120 k+40 N^{6}+66 N^{5}-44 N^{4}+2 N^{3}+152 N^{2} \\
& +80 N)] /\left[(N-2)(N+1)(N+2)^{2}(2 k+3 N+2) d(N, k)\right] \text {, } \\
& a_{4}=-\frac{12 N(N+1)(k+N)\left(10 k^{2} N+8 k^{2}+14 k N^{2}+14 k N+5 N^{3}+6 N^{2}\right)}{(N-2)(N+2)^{2}(2 k+N)(2 k+3 N+2)}, \\
& a_{5}=-\frac{24 N(N+1)(k+N)(2 k+N)}{(N-2)(N+2)(2 k+3 N+2)}, \\
& a_{6}=-\frac{24 N(N+1)(k+N)\left(3 k N+4 k+2 N^{2}+3 N\right)}{(N-2)(N+2)^{2}(2 k+3 N+2)} \text {, } \\
& a_{7}=\frac{12 N(N+1)(k+N)\left(6 k N^{2}+14 k N+8 k+5 N^{3}+14 N^{2}+10 N\right)}{(N-2)(N+2)^{2}(2 k+N)(2 k+3 N+2)}, \\
& a_{8}=\frac{24 N(N+1)(k+N)\left(3 k N+4 k+2 N^{2}+3 N\right)}{(N-2)(N+2)(2 k+N)(2 k+3 N+2)}, \\
& a_{9}=\frac{24 N(N+1)(k+N)}{(N-2)(2 k+3 N+2)}, \\
& a_{10}=\left[-6(N+1)(N+2)\left(10 k^{3} N^{2}+118 k^{3} N-60 k^{3}+25 k^{2} N^{3}+347 k^{2} N^{2}+22 k^{2} N-120 k^{2}\right.\right. \\
& \left.\left.+10 k N^{4}+299 k N^{3}+217 k N^{2}-72 k N-60 k+70 N^{4}+94 N^{3}+48 N^{2}+24 N\right)\right] / \\
& \times[(N-2)(2 k+N)(2 k+3 N+2) d(N, k)], \\
& a_{11}=\left[-6(N+1)(N+2)\left(15 k^{3} N^{2}-33 k^{3} N+60 k^{3}+50 k^{2} N^{3}-67 k^{2} N^{2}+63 k^{2} N+120 k^{2}\right.\right. \\
& \left.\left.+40 k N^{4}+6 k N^{3}-22 k N^{2}+72 k N+60 k+40 N^{4}+16 N^{3}-48 N^{2}-24 N\right)\right] / \\
& \times[(N-2)(2 k+N)(2 k+3 N+2) d(N, k)],
\end{aligned}
$$




$$
\begin{aligned}
& a_{12}=\frac{6 N(N+1)(N+2)}{(N-2)(2 k+N)(2 k+3 N+2)}, \\
& a_{13}=\left[96 k^{2}(k+N)^{2}\left(29 k^{2} N-71 k^{2}+58 k N^{2}-113 k N-71 k+25 N^{3}-42 N^{2}-42 N\right)\right] / \\
& \times[(N-2)(N+1)(2 k+3 N+2) p(N, k)], \\
& a_{14}=\left[-24(k+N)\left(1160 k^{6} N^{5}+4724 k^{6} N^{4}-1996 k^{6} N^{3}-29228 k^{6} N^{2}-30028 k^{6} N\right.\right. \\
& +12840 k^{6}+5800 k^{5} N^{6}+26080 k^{5} N^{5}+1636 k^{5} N^{4}-150572 k^{5} N^{3}-221780 k^{5} N^{2} \\
& +6268 k^{5} N+38520 k^{5}+10280 k^{4} N^{7}+55241 k^{4} N^{6}+34215 k^{4} N^{5}-285673 k^{4} N^{4} \\
& -585707 k^{4} N^{3}-155620 k^{4} N^{2}+131280 k^{4} N+38520 k^{4}+7640 k^{3} N^{8}+54988 k^{3} N^{7} \\
& +70558 k^{3} N^{6}-237134 k^{3} N^{5}-710602 k^{3} N^{4}-375346 k^{3} N^{3}+156916 k^{3} N^{2}+123644 k^{3} N \\
& +12840 k^{3}+2000 k^{2} N^{9}+25468 k^{2} N^{8}+53820 k^{2} N^{7}-75987 k^{2} N^{6}-404325 k^{2} N^{5} \\
& -324821 k^{2} N^{4}+79929 k^{2} N^{3}+140224 k^{2} N^{2}+28660 k^{2} N+4400 k N^{9}+13880 k N^{8} \\
& -1340 k N^{7}-89940 k N^{6}-106644 k N^{5}+18140 k N^{4}+65904 k N^{3}+20240 k N^{2} \\
& \left.\left.+2052 N^{8}-2052 N^{7}-8436 N^{6}+2052 N^{5}+10944 N^{4}+4560 N^{3}\right)\right] /[(N-2) N(N+1) \\
& \times(N+2)(2 k+3 N+2) d(N, k) p(N, k)], \\
& a_{15}=\left[-24(k+N)\left(1740 k^{6} N^{5}+6736 k^{6} N^{4}-8064 k^{6} N^{3}-37672 k^{6} N^{2}-18252 k^{6} N\right.\right. \\
& -12840 k^{6}+8700 k^{5} N^{6}+37020 k^{5} N^{5}-29016 k^{5} N^{4}-204048 k^{5} N^{3}-153420 k^{5} N^{2} \\
& -102828 k^{5} N-38520 k^{5}+15420 k^{4} N^{7}+78399 k^{4} N^{6}-23815 k^{4} N^{5}-420707 k^{4} N^{4} \\
& -422473 k^{4} N^{3}-292000 k^{4} N^{2}-179560 k^{4} N-38520 k^{4}+11460 k^{3} N^{8}+78632 k^{3} N^{7} \\
& +20162 k^{3} N^{6}-406866 k^{3} N^{5}-513898 k^{3} N^{4}-368274 k^{3} N^{3}-296236 k^{3} N^{2}-123644 k^{3} N \\
& -12840 k^{3}+3000 k^{2} N^{9}+37152 k^{2} N^{8}+34700 k^{2} N^{7}-186753 k^{2} N^{6}-283235 k^{2} N^{5} \\
& -197099 k^{2} N^{4}-207929 k^{2} N^{3}-140224 k^{2} N^{2}-28660 k^{2} N+6600 k N^{9}+11640 k N^{8} \\
& -36060 k N^{7}-54380 k N^{6}-22716 k N^{5}-55100 k N^{4}-65904 k N^{3}-20240 k N^{2} \\
& \left.\left.-2052 N^{8}+2052 N^{7}+8436 N^{6}-2052 N^{5}-10944 N^{4}-4560 N^{3}\right)\right] /[(N-2) N(N+1) \\
& \times(N+2)(2 k+3 N+2) d(N, k) p(N, k)], \\
& a_{16}=\frac{96 k(k+N)\left(29 k^{2} N-71 k^{2}+58 k N^{2}-113 k N-71 k+25 N^{3}-42 N^{2}-42 N\right)}{(N-2)(2 k+3 N+2) p(N, k)}, \\
& a_{17}=\frac{576(N+1)(k+N)^{2}\left(29 k^{2} N-71 k^{2}+58 k N^{2}-113 k N-71 k+25 N^{3}-42 N^{2}-42 N\right)}{(N-2)(2 k+3 N+2) p(N, k)} \text {, } \\
& a_{18}=\left[2 8 8 k ( N + 2 ) ( k + N ) ^ { 2 } \left(29 k^{2} N-71 k^{2}+58 k N^{2}-113 k N-71 k+25 N^{3}-42 N^{2}\right.\right. \\
& -42 N)] /[(N-2)(2 k+N)(2 k+3 N+2) p(N, k)] \text {, } \\
& a_{19}=-\frac{288(N+1)(k+N)\left(29 k^{2} N-71 k^{2}+58 k N^{2}-113 k N-71 k+25 N^{3}-42 N^{2}-42 N\right)}{(N-2)(2 k+3 N+2) p(N, k)}, \\
& a_{20}=\left[-96 k(N+2)(k+N)\left(29 k^{2} N-71 k^{2}+58 k N^{2}-113 k N-71 k+25 N^{3}-42 N^{2}\right.\right. \\
& -42 N)] /[(N-2)(2 k+N)(2 k+3 N+2) p(N, k)], \\
& a_{21}=\left[-576(N+1)(N+2)(k+N)^{2}\left(29 k^{2} N-71 k^{2}+58 k N^{2}-113 k N-71 k+25 N^{3}\right.\right. \\
& \left.\left.-42 N^{2}-42 N\right)\right] /[(N-2)(2 k+N)(2 k+3 N+2) p(N, k)], \\
& a_{22}=\left[2 4 ( N + 1 ) ( N + 2 ) \left(1670 k^{5} N^{3}+936 k^{5} N^{2}-9554 k^{5} N-6420 k^{5}+8315 k^{4} N^{4}\right.\right. \\
& +7366 k^{4} N^{3}-47129 k^{4} N^{2}-42640 k^{4} N-19260 k^{4}+14720 k^{3} N^{5}+23946 k^{3} N^{4} \\
& -92706 k^{3} N^{3}-106362 k^{3} N^{2}-60890 k^{3} N-19260 k^{3}+11040 k^{2} N^{6}+35816 k^{2} N^{5} \\
& -87917 k^{2} N^{4}-132278 k^{2} N^{3}-65641 k^{2} N^{2}-32076 k^{2} N-6420 k^{2}+3000 k N^{7} \\
& +24900 k N^{6}-39648 k N^{5}-85680 k N^{4}-28068 k N^{3}-4608 k N^{2}-4272 k N+6600 N^{7}
\end{aligned}
$$




$$
\begin{aligned}
& \left.\left.-6540 N^{6}-23544 N^{5}-4932 N^{4}+8208 N^{3}+2736 N^{2}\right)\right] /[(N-2) N(2 k+N) \\
& \times(2 k+3 N+2) d(N, k) p(N, k)], \\
& a_{23}=\left[2 4 ( N + 1 ) ( N + 2 ) \left(1230 k^{5} N^{3}+1824 k^{5} N^{2}-14586 k^{5} N+6420 k^{5}+6185 k^{4} N^{4}\right.\right. \\
& +12234 k^{4} N^{3}-68051 k^{4} N^{2}-5640 k^{4} N+19260 k^{4}+10980 k^{3} N^{5}+32594 k^{3} N^{4} \\
& -117914 k^{3} N^{3}-81238 k^{3} N^{2}+36750 k^{3} N+19260 k^{3}+8060 k^{2} N^{6}+40504 k^{2} N^{5} \\
& -88523 k^{2} N^{4}-135042 k^{2} N^{3}-4019 k^{2} N^{2}+32076 k^{2} N+6420 k^{2}+2000 k N^{7}+22720 k N^{6} \\
& -24692 k N^{5}-79280 k N^{4}-35932 k N^{3}+4608 k N^{2}+4272 k N+4400 N^{7}-940 N^{6} \\
& \left.\left.-13416 N^{5}-13548 N^{4}-8208 N^{3}-2736 N^{2}\right)\right] /[(N-2) N(2 k+N)(2 k+3 N+2) \\
& \times d(N, k) p(N, k)], \\
& a_{24}=\left[-96(N+1)(N+2)\left(29 k^{2} N-71 k^{2}+58 k N^{2}-113 k N-71 k+25 N^{3}-42 N^{2}\right.\right. \\
& -42 N)] /[(N-2)(2 k+N)(2 k+3 N+2) p(N, k)], \\
& a_{25}=\left[-6 k(k+N)(2 k+N)\left(5 k^{2} N^{2}+102 k^{2} N+177 k^{2}+10 k N^{3}+113 k N^{2}+168 k N-15 k\right.\right. \\
& \left.\left.+6 N^{3}-4 N^{2}-10 N\right)\right] /[(N+1)(N+2) d(N, k)], \\
& a_{26}=\left[-6(k+N)\left(28 k^{7} N^{5}+9980 k^{7} N^{4}-15092 k^{7} N^{3}-76428 k^{7} N^{2}+47656 k^{7} N+79840 k^{7}\right.\right. \\
& -84 k^{6} N^{6}+67148 k^{6} N^{5}-38204 k^{6} N^{4}-518708 k^{6} N^{3}-46512 k^{6} N^{2}+493272 k^{6} N \\
& +184112 k^{6}-1085 k^{5} N^{7}+183099 k^{5} N^{6}+87751 k^{5} N^{5}-1308927 k^{5} N^{4}-1204222 k^{5} N^{3} \\
& +301304 k^{5} N^{2}+370520 k^{5} N+15088 k^{5}-2625 k^{4} N^{8}+260028 k^{4} N^{7}+439584 k^{4} N^{6} \\
& -1470698 k^{4} N^{5}-3320543 k^{4} N^{4}-2793274 k^{4} N^{3}-1868128 k^{4} N^{2}-979368 k^{4} N \\
& -202800 k^{4}-2408 k^{3} N^{9}+203242 k^{3} N^{8}+654939 k^{3} N^{7}-531605 k^{3} N^{6}-3877529 k^{3} N^{5} \\
& -6792005 k^{3} N^{4}-6991174 k^{3} N^{3}-4226988 k^{3} N^{2}-1240000 k^{3} N-113616 k^{3}-756 k^{2} N^{10} \\
& +83104 k^{2} N^{9}+463719 k^{2} N^{8}+321718 k^{2} N^{7}-2089910 k^{2} N^{6}-6378852 k^{2} N^{5} \\
& -8686421 k^{2} N^{4}-6360778 k^{2} N^{3}-2357168 k^{2} N^{2}-335728 k^{2} N+13896 k N^{10} \\
& +157824 k N^{9}+322196 k N^{8}-424092 k N^{7}-2686788 k N^{6}-4661748 k N^{5}-4028904 k N^{4} \\
& -1759680 k N^{3}-308096 k N^{2}+20652 N^{10}+73512 N^{9}-1656 N^{8}-420384 N^{7}-912084 N^{6} \\
& \left.\left.-899496 N^{5}-439488 N^{4}-86208 N^{3}\right)\right] /[(N-2)(N+1)(N+2)(k+N+1) \\
& \times(2 k+3 N+2) d(N, k) p(N, k)], \\
& a_{27}=\left[3 ( k + N ) \left(1764 k^{6} N^{7}+9372 k^{6} N^{6}-7844 k^{6} N^{5}-183340 k^{6} N^{4}-494352 k^{6} N^{3}\right.\right. \\
& -342560 k^{6} N^{2}+74144 k^{6} N+205440 k^{6}+8904 k^{5} N^{8}+31028 k^{5} N^{7}-76772 k^{5} N^{6} \\
& -809716 k^{5} N^{5}-2334324 k^{5} N^{4}-2397008 k^{5} N^{3}-472096 k^{5} N^{2}+1263328 k^{5} N+616320 k^{5} \\
& +15071 k^{4} N^{9}+12703 k^{4} N^{8}-223001 k^{4} N^{7}-1151167 k^{4} N^{6}-3856922 k^{4} N^{5} \\
& -5914876 k^{4} N^{4}-3482104 k^{4} N^{3}+2027248 k^{4} N^{2}+2762784 k^{4} N+616320 k^{4}+9884 k^{3} N^{10} \\
& -44374 k^{3} N^{9}-268638 k^{3} N^{8}-428282 k^{3} N^{7}-2398410 k^{3} N^{6}-6576776 k^{3} N^{5} \\
& -6927340 k^{3} N^{4}+117120 k^{3} N^{3}+4306112 k^{3} N^{2}+2032160 k^{3} N+205440 k^{3}+2492 k^{2} N^{11} \\
& -48100 k^{2} N^{10}-131589 k^{2} N^{9}+337475 k^{2} N^{8}-52573 k^{2} N^{7}-3524867 k^{2} N^{6} \\
& -6036286 k^{2} N^{5}-1801944 k^{2} N^{4}+3166328 k^{2} N^{3}+2473168 k^{2} N^{2}+458560 k^{2} N \\
& -13624 k N^{11}-3348 k N^{10}+313836 k N^{9}+404028 k N^{8}-937572 k N^{7}-2354008 k N^{6} \\
& -1046816 k N^{5}+1257632 k N^{4}+1312320 k N^{3}+323840 k N^{2}+9084 N^{11}+65436 N^{10} \\
& \left.\left.+84780 N^{9}-111084 N^{8}-314616 N^{7}-114528 N^{6}+248544 N^{5}+260928 N^{4}+72960 N^{3}\right)\right] / \\
& \times\left[(N-2) N(N+1)(N+2)^{2}(2 k+3 N+2) d(N, k) p(N, k)\right],
\end{aligned}
$$




$$
\begin{aligned}
& a_{28}=\left[-3(k+N)(2 k+N)\left(138 k^{4} N^{4}-180 k^{4} N^{3}-862 k^{4} N^{2}+1120 k^{4} N+1088 k^{4}+621 k^{3} N^{5}\right.\right. \\
& -396 k^{3} N^{4}-4419 k^{3} N^{3}+2454 k^{3} N^{2}+8256 k^{3} N+3264 k^{3}+897 k^{2} N^{6}+52 k^{2} N^{5} \\
& -7441 k^{2} N^{4}-1182 k^{2} N^{3}+14934 k^{2} N^{2}+12528 k^{2} N+2720 k^{2}+414 k N^{7}+307 k N^{6} \\
& -4715 k N^{5}-4213 k N^{4}+8413 k N^{3}+12674 k N^{2}+5200 k N+544 k-30 N^{7}-878 N^{6} \\
& \left.\left.-1110 N^{5}+1542 N^{4}+3244 N^{3}+1632 N^{2}+192 N\right)\right] /[(N-2)(N+1)(N+2) \\
& \times(k+N+1)(2 k+3 N+2) d(N, k)], \\
& a_{29}=\left[3 ( k + N ) ( 2 k + N ) \left(110 k^{4} N^{5}+1172 k^{4} N^{4}-26 k^{4} N^{3}-4976 k^{4} N^{2}-2544 k^{4} N-960 k^{4}\right.\right. \\
& +375 k^{3} N^{6}+4348 k^{3} N^{5}+1951 k^{3} N^{4}-18462 k^{3} N^{3}-18440 k^{3} N^{2}-8336 k^{3} N-2880 k^{3} \\
& +355 k^{2} N^{7}+5468 k^{2} N^{6}+5061 k^{2} N^{5}-22922 k^{2} N^{4}-33494 k^{2} N^{3}-18032 k^{2} N^{2} \\
& -9136 k^{2} N-2880 k^{2}+90 k N^{8}+2425 k N^{7}+3551 k N^{6}-10535 k N^{5}-20129 k N^{4} \\
& -10402 k N^{3}-5048 k N^{2}-3984 k N-960 k+198 N^{8}+342 N^{7}-1554 N^{6}-2526 N^{5} \\
& \left.\left.+404 N^{4}+1008 N^{3}-864 N^{2}-640 N\right)\right] /[(N-2) N(N+1)(N+2)(k+N+1) \\
& \times(2 k+3 N+2) d(N, k)], \\
& a_{30}=\left[6 ( k + N ) \left(40 k^{4} N^{4}-120 k^{4} N^{3}-168 k^{4} N^{2}+888 k^{4} N+512 k^{4}+160 k^{3} N^{5}-360 k^{3} N^{4}\right.\right. \\
& -816 k^{3} N^{3}+2840 k^{3} N^{2}+2848 k^{3} N+480 k^{3}+195 k^{2} N^{6}-338 k^{2} N^{5}-1165 k^{2} N^{4} \\
& +3252 k^{2} N^{3}+4804 k^{2} N^{2}+1368 k^{2} N-32 k^{2}+70 k N^{7}-161 k N^{6}-492 k N^{5}+2011 k N^{4} \\
& \left.\left.+3316 k N^{3}+932 k N^{2}-208 k N-38 N^{7}-20 N^{6}+546 N^{5}+808 N^{4}+184 N^{3}-96 N^{2}\right)\right] / \\
& \times[(N-2)(N+2)(k+N+1)(2 k+3 N+2) d(N, k)], \\
& a_{31}=\left[-6(k+N)\left(280 k^{6} N^{5}-16056 k^{6} N^{4}+10008 k^{6} N^{3}+140488 k^{6} N^{2}+32240 k^{6} N\right.\right. \\
& -120304 k^{6}+1540 k^{5} N^{6}-98252 k^{5} N^{5}+3388 k^{5} N^{4}+893796 k^{5} N^{3}+724848 k^{5} N^{2} \\
& -512776 k^{5} N-331808 k^{5}+3045 k^{4} N^{7}-230305 k^{4} N^{6}-120541 k^{4} N^{5}+2176321 k^{4} N^{4} \\
& +3000692 k^{4} N^{3}-291612 k^{4} N^{2}-1552216 k^{4} N-448224 k^{4}+2660 k^{3} N^{8}-257834 k^{3} N^{7} \\
& -275698 k^{3} N^{6}+2576254 k^{3} N^{5}+5096606 k^{3} N^{4}+1182380 k^{3} N^{3}-2949896 k^{3} N^{2} \\
& -2066968 k^{3} N-382240 k^{3}+980 k^{2} N^{9}-137796 k^{2} N^{8}-234303 k^{2} N^{7}+1547899 k^{2} N^{6} \\
& +4172067 k^{2} N^{5}+1932785 k^{2} N^{4}-3114796 k^{2} N^{3}-3786148 k^{2} N^{2}-1403128 k^{2} N \\
& -145520 k^{2}-28456 k N^{9}-83612 k N^{8}+431852 k N^{7}+1650396 k N^{6}+1127164 k N^{5} \\
& -1812528 k N^{4}-3230608 k N^{3}-1797664 k N^{2}-343360 k N-11436 N^{9}+34044 N^{8} \\
& \left.\left.+262476 N^{7}+278244 N^{6}-417840 N^{5}-1059984 N^{4}-781536 N^{3}-200640 N^{2}\right)\right] / \\
& \times[(N-2)(N+2)(k+N+1)(2 k+3 N+2) d(N, k) p(N, k)], \\
& a_{32}=\left[6 ( k + N ) \left(80 k^{4} N^{4}-32 k^{4} N^{3}-1056 k^{4} N^{2}-928 k^{4} N+1168 k^{4}+300 k^{3} N^{5}-16 k^{3} N^{4}\right.\right. \\
& -3980 k^{3} N^{3}-4888 k^{3} N^{2}+3144 k^{3} N+2064 k^{3}+335 k^{2} N^{6}+78 k^{2} N^{5}-4681 k^{2} N^{4} \\
& -7692 k^{2} N^{3}+2076 k^{2} N^{2}+5072 k^{2} N+1168 k^{2}+110 k N^{7}-29 k N^{6}-1952 k N^{5} \\
& -3641 k N^{4}+840 k N^{3}+3844 k N^{2}+1736 k N+272 k-46 N^{7}-220 N^{6}-374 N^{5}+320 N^{4} \\
& \left.\left.+1176 N^{3}+1008 N^{2}+352 N\right)\right] /[(N-2)(N+2)(k+N+1)(2 k+3 N+2) d(N, k)], \\
& a_{33}=\left[-6(k+N)\left(1680 k^{7} N^{6}-18016 k^{7} N^{5}-137568 k^{7} N^{4}-158976 k^{7} N^{3}+362000 k^{7} N^{2}\right.\right. \\
& +847840 k^{7} N+367936 k^{7}+10080 k^{6} N^{7}-152464 k^{6} N^{6}-1141312 k^{6} N^{5}-1483104 k^{6} N^{4} \\
& +3199776 k^{6} N^{3}+9279728 k^{6} N^{2}+6833344 k^{6} N+1526528 k^{6}+22470 k^{5} N^{8}-506358 k^{5} N^{7} \\
& -3834166 k^{5} N^{6}-5740530 k^{5} N^{5}+10503784 k^{5} N^{4}+38139904 k^{5} N^{3}+37670784 k^{5} N^{2} \\
& +14508128 k^{5} N+1673472 k^{5}+23205 k^{4} N^{9}-848375 k^{4} N^{8}-6738131 k^{4} N^{7}
\end{aligned}
$$




$$
\begin{aligned}
& -11948949 k^{4} N^{6}+16361850 k^{4} N^{5}+78904344 k^{4} N^{4}+97622512 k^{4} N^{3}+51571600 k^{4} N^{2} \\
& +10408288 k^{4} N+239104 k^{4}+11340 k^{3} N^{10}-761834 k^{3} N^{9}-6657412 k^{3} N^{8} \\
& -14318048 k^{3} N^{7}+12041840 k^{3} N^{6}+89502858 k^{3} N^{5}+135816408 k^{3} N^{4}+91329120 k^{3} N^{3} \\
& +26271120 k^{3} N^{2}+1532224 k^{3} N-275776 k^{3}+2100 k^{2} N^{11}-350556 k^{2} N^{10}-3650887 k^{2} N^{9} \\
& -9812615 k^{2} N^{8}+2602561 k^{2} N^{7}+55433623 k^{2} N^{6}+103916830 k^{2} N^{5}+86470824 k^{2} N^{4} \\
& +33199440 k^{2} N^{3}+3984928 k^{2} N^{2}-353440 k^{2} N-64872 k N^{11}-998436 k N^{10} \\
& -3549284 k N^{9}-1346868 k N^{8}+16948828 k N^{7}+40948040 k N^{6}+42003808 k N^{5} \\
& +20918528 k N^{4}+4254336 k N^{3}+71552 k N^{2}-96972 N^{11}-520140 N^{10}-595836 N^{9} \\
& \left.\left.+1833180 N^{8}+6422712 N^{7}+8244384 N^{6}+5247552 N^{5}+1577088 N^{4}+157056 N^{3}\right)\right] / \\
& \times\left[(N-2)(N+2)^{2}(k+N+1)(2 k+N)(2 k+3 N+2) d(N, k) p(N, k)\right], \\
& a_{34}=\left[6 ( k + N ) \left(120 k^{4} N^{5}+344 k^{4} N^{4}-648 k^{4} N^{3}-1880 k^{4} N^{2}+1264 k^{4} N+3424 k^{4}\right.\right. \\
& +440 k^{3} N^{6}+1420 k^{3} N^{5}-2008 k^{3} N^{4}-8516 k^{3} N^{3}+520 k^{3} N^{2}+13568 k^{3} N+5216 k^{3} \\
& +475 k^{2} N^{7}+1840 k^{2} N^{6}-1681 k^{2} N^{5}-11834 k^{2} N^{4}-5716 k^{2} N^{3}+14032 k^{2} N^{2}+10768 k^{2} N \\
& +1248 k^{2}+150 k N^{8}+731 k N^{7}-386 k N^{6}-5129 k N^{5}-5002 k N^{4}+2152 k N^{3}+1776 k N^{2} \\
& -1472 k N-544 k+42 N^{8}+40 N^{7}-286 N^{6}-780 N^{5}-1616 N^{4}-2976 N^{3}-2560 N^{2} \\
& -704 N)] /\left[(N-2)(N+2)^{2}(k+N+1)(2 k+3 N+2) d(N, k)\right], \\
& a_{35}=\left[-6(k+N)\left(1400 k^{7} N^{6}+16552 k^{7} N^{5}+49800 k^{7} N^{4}-54536 k^{7} N^{3}-355040 k^{7} N^{2}\right.\right. \\
& -281216 k^{7} N+23040 k^{7}+9660 k^{6} N^{7}+129604 k^{6} N^{6}+456388 k^{6} N^{5}-159988 k^{6} N^{4} \\
& -2903632 k^{6} N^{3}-3759584 k^{6} N^{2}-1014720 k^{6} N+260864 k^{6}+25550 k^{5} N^{8}+406618 k^{5} N^{7} \\
& +1707322 k^{5} N^{6}+472718 k^{5} N^{5}-9573936 k^{5} N^{4}-17420336 k^{5} N^{3}-9085568 k^{5} N^{2} \\
& +481952 k^{5} N+877184 k^{5}+32165 k^{4} N^{9}+650705 k^{4} N^{8}+3312645 k^{4} N^{7}+2838979 k^{4} N^{6} \\
& -15910142 k^{4} N^{5}-39616552 k^{4} N^{4}-30318608 k^{4} N^{3}-3526128 k^{4} N^{2}+4227680 k^{4} N \\
& +1063936 k^{4}+19180 k^{3} N^{10}+557942 k^{3} N^{9}+3547076 k^{3} N^{8}+5152928 k^{3} N^{7} \\
& -13677080 k^{3} N^{6}-48638126 k^{3} N^{5}-50275896 k^{3} N^{4}-14542440 k^{3} N^{3}+6419200 k^{3} N^{2} \\
& +3974752 k^{3} N+424576 k^{3}+4340 k^{2} N^{11}+243108 k^{2} N^{10}+2058345 k^{2} N^{9}+4414657 k^{2} N^{8} \\
& -5344003 k^{2} N^{7}-32154533 k^{2} N^{6}-43762318 k^{2} N^{5}-20922244 k^{2} N^{4}+2409472 k^{2} N^{3} \\
& +4756976 k^{2} N^{2}+962528 k^{2} N+42200 k N^{11}+579740 k N^{10}+1805340 k N^{9}-335524 k N^{8} \\
& -10393396 k N^{7}-18936904 k N^{6}-13222992 k N^{5}-1794048 k N^{4}+1928384 k N^{3} \\
& +646272 k N^{2}+55860 N^{11}+280212 N^{10}+216372 N^{9}-1195860 N^{8}-3176952 N^{7} \\
& \left.\left.-3101712 N^{6}-1138176 N^{5}+83904 N^{4}+109440 N^{3}\right)\right] /\left[(N-2)(N+2)^{2}(k+N+1)\right. \\
& \times(2 k+N)(2 k+3 N+2) d(N, k) p(N, k)], \\
& a_{36}=\left[-3(N+1)\left(60 k^{5} N^{4}-308 k^{5} N^{3}-160 k^{5} N^{2}+1504 k^{5} N+240 k^{4} N^{5}-1428 k^{4} N^{4}\right.\right. \\
& -756 k^{4} N^{3}+7840 k^{4} N^{2}+736 k^{4} N-3840 k^{4}+315 k^{3} N^{6}-2543 k^{3} N^{5}-746 k^{3} N^{4} \\
& +15900 k^{3} N^{3}+4048 k^{3} N^{2}-13856 k^{3} N-11520 k^{3}+165 k^{2} N^{7}-2175 k^{2} N^{6}+644 k^{2} N^{5} \\
& +15416 k^{2} N^{4}+4516 k^{2} N^{3}-13888 k^{2} N^{2}-23456 k^{2} N-11520 k^{2}+30 k N^{8}-863 k N^{7} \\
& +1464 k N^{6}+6911 k N^{5}-2494 k N^{4}-6040 k N^{3}-5328 k N^{2}-8832 k N-3840 k-126 N^{8} \\
& \left.\left.+640 N^{7}+1290 N^{6}-3236 N^{5}-3344 N^{4}+4256 N^{3}+5376 N^{2}+1536 N\right)\right] /[(N-2) N \\
& \times(k+N+1)(2 k+N)(2 k+3 N+2) d(N, k)], \\
& a_{37}=\left[3 ( N + 1 ) \left(784 k^{6} N^{4}-8912 k^{6} N^{3}+18368 k^{6} N^{2}+61232 k^{6} N-24432 k^{6}+4494 k^{5} N^{5}\right.\right.
\end{aligned}
$$




$$
\begin{aligned}
& -56220 k^{5} N^{4}+77494 k^{5} N^{3}+439928 k^{5} N^{2}+49256 k^{5} N-91664 k^{5}+9121 k^{4} N^{6} \\
& -136172 k^{4} N^{5}+108325 k^{4} N^{4}+1139978 k^{4} N^{3}+654248 k^{4} N^{2}-165744 k^{4} N-110032 k^{4} \\
& +7644 k^{3} N^{7}-158302 k^{3} N^{6}+29770 k^{3} N^{5}+1370726 k^{3} N^{4}+1530866 k^{3} N^{3}+244264 k^{3} N^{2} \\
& -245592 k^{3} N-42800 k^{3}+2212 k^{2} N^{8}-88688 k^{2} N^{7}-66315 k^{2} N^{6}+770768 k^{2} N^{5} \\
& +1563901 k^{2} N^{4}+789102 k^{2} N^{3}-138440 k^{2} N^{2}-91824 k^{2} N-19208 k N^{8}-64748 k N^{7} \\
& +156824 k N^{6}+758660 k N^{5}+637384 k N^{4}+7872 k N^{3}-73216 k N^{2}-17820 N^{8}-4416 N^{7} \\
& \left.\left.+145716 N^{6}+167928 N^{5}+10080 N^{4}-25536 N^{3}\right)\right] /[(N-2)(2 k+N)(2 k+3 N+2) \\
& \times d(N, k) p(N, k)], \\
& a_{38}=\left[-3(N+1)\left(116 k^{5} N^{3}-476 k^{5} N^{2}-704 k^{5} N+1088 k^{5}+640 k^{4} N^{4}-2252 k^{4} N^{3}\right.\right. \\
& -5084 k^{4} N^{2}+5504 k^{4} N+5440 k^{4}+1305 k^{3} N^{5}-3797 k^{3} N^{4}-12518 k^{3} N^{3}+7628 k^{3} N^{2} \\
& +21248 k^{3} N+6528 k^{3}+1175 k^{2} N^{6}-2693 k^{2} N^{5}-13116 k^{2} N^{4}+1392 k^{2} N^{3}+26700 k^{2} N^{2} \\
& +17600 k^{2} N+2176 k^{2}+394 k N^{7}-725 k N^{6}-5192 k N^{5}-2259 k N^{4}+11094 k N^{3} \\
& \left.\left.+12416 k N^{2}+3328 k N-74 N^{7}-256 N^{6}-258 N^{5}+564 N^{4}+896 N^{3}+256 N^{2}\right)\right] / \\
& \times[(N-2)(k+N+1)(2 k+N)(2 k+3 N+2) d(N, k)], \\
& a_{39}=\left[6 ( N + 1 ) \left(392 k^{7} N^{4}+8120 k^{7} N^{3}+30568 k^{7} N^{2}-37688 k^{7} N-118128 k^{7}+2604 k^{6} N^{5}\right.\right. \\
& +56100 k^{6} N^{4}+231620 k^{6} N^{3}-162756 k^{6} N^{2}-986912 k^{6} N-492432 k^{6}+6545 k^{5} N^{6} \\
& +156248 k^{5} N^{5}+742237 k^{5} N^{4}-139386 k^{5} N^{3}-3227336 k^{5} N^{2}-3091736 k^{5} N-710320 k^{5} \\
& +7665 k^{4} N^{7}+223735 k^{4} N^{6}+1292109 k^{4} N^{5}+419449 k^{4} N^{4}-5413350 k^{4} N^{3} \\
& -7793520 k^{4} N^{2}-3445416 k^{4} N-415856 k^{4}+4088 k^{3} N^{8}+172238 k^{3} N^{7}+1309507 k^{3} N^{6} \\
& +1111550 k^{3} N^{5}-5028613 k^{3} N^{4}-10097078 k^{3} N^{3}-6453952 k^{3} N^{2}-1470320 k^{3} N \\
& -79840 k^{3}+756 k^{2} N^{9}+66812 k^{2} N^{8}+767677 k^{2} N^{7}+1069161 k^{2} N^{6}-2579087 k^{2} N^{5} \\
& -7097909 k^{2} N^{4}-5792518 k^{2} N^{3}-1818252 k^{2} N^{2}-167416 k^{2} N+10104 k N^{9}+241368 k N^{8} \\
& +468452 k N^{7}-689864 k N^{6}-2584068 k N^{5}-2447784 k N^{4}-876176 k N^{3}-85408 k N^{2} \\
& \left.\left.+32148 N^{9}+77844 N^{8}-83052 N^{7}-390708 N^{6}-377496 N^{5}-111408 N^{4}+4128 N^{3}\right)\right] / \\
& \times[(N-2)(k+N+1)(2 k+N)(2 k+3 N+2) d(N, k) p(N, k)], \\
& a_{40}=\frac{6(N+1)(N+2)\left(5 k^{2} N-15 k^{2}+10 k N^{2}-9 k N+177 k+6 N^{2}+102 N\right)}{(2 k+N) d(N, k)}, \\
& a_{41}=\left[1 2 k ( k + N ) \left(20 k^{4} N^{2}-140 k^{4} N+240 k^{4}+80 k^{3} N^{3}-520 k^{3} N^{2}+680 k^{3} N+480 k^{3}\right.\right. \\
& +97 k^{2} N^{4}-663 k^{2} N^{3}+660 k^{2} N^{2}+1160 k^{2} N+240 k^{2}+34 k N^{5}-349 k N^{4}+297 k N^{3} \\
& \left.\left.+920 k N^{2}+340 k N-66 N^{5}+54 N^{4}+240 N^{3}+120 N^{2}\right)\right] /[(N-2)(2 k+3 N+2) p(N, k)], \\
& a_{42}=\left[-12 k(k+N)\left(220 k^{6} N^{5}+2268 k^{6} N^{4}+16220 k^{6} N^{3}-11004 k^{6} N^{2}-60936 k^{6} N\right.\right. \\
& +12240 k^{6}+1320 k^{5} N^{6}+12924 k^{5} N^{5}+82236 k^{5} N^{4}-32532 k^{5} N^{3}-311076 k^{5} N^{2} \\
& -27192 k^{5} N+36720 k^{5}+2820 k^{4} N^{7}+27611 k^{4} N^{6}+163705 k^{4} N^{5}-31147 k^{4} N^{4} \\
& -605101 k^{4} N^{3}-154156 k^{4} N^{2}+161604 k^{4} N+36720 k^{4}+2480 k^{3} N^{8}+27284 k^{3} N^{7} \\
& +158370 k^{3} N^{6}-16750 k^{3} N^{5}-562158 k^{3} N^{4}-121966 k^{3} N^{3}+366676 k^{3} N^{2}+161040 k^{3} N \\
& +12240 k^{3}+720 k^{2} N^{9}+12212 k^{2} N^{8}+72264 k^{2} N^{7}-23945 k^{2} N^{6}-248831 k^{2} N^{5} \\
& +91009 k^{2} N^{4}+459263 k^{2} N^{3}+249320 k^{2} N^{2}+33180 k^{2} N+1968 k N^{9}+10176 k N^{8} \\
& -25460 k N^{7}-41604 k N^{6}+140596 k N^{5}+282372 k N^{4}+162400 k N^{3}+28560 k N^{2} \\
& \left.\left.-1152 N^{9}-7956 N^{8}-60 N^{7}+41220 N^{6}+64476 N^{5}+37920 N^{4}+7920 N^{3}\right)\right] /[(N-2) \\
& \times(N+1)(N+2)(2 k+3 N+2) d(N, k) p(N, k)],
\end{aligned}
$$




$$
\begin{aligned}
& a_{43}=\left[-3(k+N)\left(2620 k^{6} N^{7}+26236 k^{6} N^{6}+63996 k^{6} N^{5}-112412 k^{6} N^{4}-543112 k^{6} N^{3}\right.\right. \\
& -362960 k^{6} N^{2}+177120 k^{6} N+130560 k^{6}+14460 k^{5} N^{8}+138372 k^{5} N^{7}+339876 k^{5} N^{6} \\
& -533524 k^{5} N^{5}-2889680 k^{5} N^{4}-2314752 k^{5} N^{3}+1097120 k^{5} N^{2}+1605760 k^{5} N \\
& +391680 k^{5}+27885 k^{4} N^{9}+268627 k^{4} N^{8}+713991 k^{4} N^{7}-914923 k^{4} N^{6}-6097016 k^{4} N^{5} \\
& -5500484 k^{4} N^{4}+3582440 k^{4} N^{3}+6954080 k^{4} N^{2}+3034080 k^{4} N+391680 k^{4}+21860 k^{3} N^{10} \\
& +235542 k^{3} N^{9}+730862 k^{3} N^{8}-731386 k^{3} N^{7}-6477154 k^{3} N^{6}-6103364 k^{3} N^{5} \\
& +6939512 k^{3} N^{4}+14493168 k^{3} N^{3}+8536480 k^{3} N^{2}+1959360 k^{3} N+130560 k^{3}+5940 k^{2} N^{11} \\
& +89116 k^{2} N^{10}+351593 k^{2} N^{9}-344817 k^{2} N^{8}-3702509 k^{2} N^{7}-3203103 k^{2} N^{6} \\
& +7528196 k^{2} N^{5}+15700872 k^{2} N^{4}+11056848 k^{2} N^{3}+3347120 k^{2} N^{2}+353920 k^{2} N \\
& +10680 k N^{11}+60692 k N^{10}-154756 k N^{9}-1177780 k N^{8}-691044 k N^{7}+4178704 k N^{6} \\
& +8510880 k N^{5}+6657344 k N^{4}+2347840 k N^{3}+304640 k N^{2}-1260 N^{11}-43764 N^{10} \\
& -182292 N^{9}-35868 N^{8}+900240 N^{7}+1802016 N^{6}+1502016 N^{5}+580800 N^{4} \\
& \left.\left.+84480 N^{3}\right)\right] /\left[(N-2)(N+1)(N+2)^{2}(2 k+3 N+2) d(N, k) p(N, k)\right], \\
& a_{44}=\left[-12(N+1)(k+N)\left(20 k^{4} N^{2}-140 k^{4} N+240 k^{4}+115 k^{3} N^{3}-765 k^{3} N^{2}+1100 k^{3} N\right.\right. \\
& +480 k^{3}+195 k^{2} N^{4}-1435 k^{2} N^{3}+2182 k^{2} N^{2}+1152 k^{2} N+240 k^{2}+90 k N^{5}-985 k N^{4} \\
& \left.\left.+2044 k N^{3}+631 k N^{2}-88 k N-210 N^{5}+720 N^{4}-6 N^{3}-336 N^{2}\right)\right] /[(N-2) \\
& \times(2 k+3 N+2) p(N, k)], \\
& a_{45}=\left[6 ( k + N ) \left(468 k^{7} N^{6}-2100 k^{7} N^{5}-82852 k^{7} N^{4}-35468 k^{7} N^{3}+374368 k^{7} N^{2}\right.\right. \\
& +251792 k^{7} N+75840 k^{7}+3696 k^{6} N^{7}-2012 k^{6} N^{6}-516100 k^{6} N^{5}-516244 k^{6} N^{4} \\
& +2236500 k^{6} N^{3}+2822784 k^{6} N^{2}+1344592 k^{6} N+303360 k^{6}+11115 k^{5} N^{8}+31369 k^{5} N^{7} \\
& -1303945 k^{5} N^{6}-2141755 k^{5} N^{5}+5125466 k^{5} N^{4}+10391038 k^{5} N^{3}+7137992 k^{5} N^{2} \\
& +2645816 k^{5} N+455040 k^{5}+15675 k^{4} N^{9}+98058 k^{4} N^{8}-1702470 k^{4} N^{7}-4108438 k^{4} N^{6} \\
& +5521993 k^{4} N^{5}+18212204 k^{4} N^{4}+16761034 k^{4} N^{3}+8306672 k^{4} N^{2}+2446024 k^{4} N \\
& +303360 k^{4}+10152 k^{3} N^{10}+114482 k^{3} N^{9}-1222297 k^{3} N^{8}-4146939 k^{3} N^{7} \\
& +2596823 k^{3} N^{6}+16947359 k^{3} N^{5}+20080134 k^{3} N^{4}+12365574 k^{3} N^{3}+4852232 k^{3} N^{2} \\
& +1074008 k^{3} N+75840 k^{3}+2364 k^{2} N^{11}+57944 k^{2} N^{10}-482005 k^{2} N^{9}-2237644 k^{2} N^{8} \\
& +153636 k^{2} N^{7}+8477520 k^{2} N^{6}+12681099 k^{2} N^{5}+9344316 k^{2} N^{4}+4479258 k^{2} N^{3} \\
& +1373216 k^{2} N^{2}+181000 k^{2} N+10536 k N^{11}-101248 k N^{10}-604332 k N^{9}-238644 k N^{8} \\
& +2132108 k N^{7}+3987884 k N^{6}+3440456 k N^{5}+1944024 k N^{4}+751328 k N^{3}+138080 k N^{2} \\
& -9828 N^{11}-63840 N^{10}-51120 N^{9}+208560 N^{8}+490476 N^{7}+494160 N^{6}+326568 N^{5} \\
& \left.\left.+151296 N^{4}+34080 N^{3}\right)\right] /[(N-2)(N+1)(N+2)(k+N+1)(2 k+3 N+2) \\
& \times d(N, k) p(N, k)], \\
& a_{46}=\left[6 ( k + N ) \left(1256 k^{8} N^{7}+22952 k^{8} N^{6}-14744 k^{8} N^{5}-290984 k^{8} N^{4}-147760 k^{8} N^{3}\right.\right. \\
& +799584 k^{8} N^{2}+850240 k^{8} N+195840 k^{8}+8580 k^{7} N^{8}+188300 k^{7} N^{7}+114524 k^{7} N^{6} \\
& -1939436 k^{7} N^{5}-2426320 k^{7} N^{4}+4268400 k^{7} N^{3}+8530304 k^{7} N^{2}+4627584 k^{7} N \\
& +783360 k^{7}+23026 k^{6} N^{9}+655646 k^{6} N^{8}+1239430 k^{6} N^{7}-4695070 k^{6} N^{6} \\
& -11912464 k^{6} N^{5}+2898856 k^{6} N^{4}+25688576 k^{6} N^{3}+23977792 k^{6} N^{2}+8791296 k^{6} N \\
& +1175040 k^{6}+30995 k^{5} N^{10}+1253145 k^{5} N^{9}+4004649 k^{5} N^{8}-4086181 k^{5} N^{7} \\
& -27397424 k^{5} N^{6}-23829864 k^{5} N^{5}+19724976 k^{5} N^{4}+44324592 k^{5} N^{3}+28204416 k^{5} N^{2}
\end{aligned}
$$




$$
\begin{aligned}
& +7809280 k^{5} N+783360 k^{5}+21999 k^{4} N^{11}+1413912 k^{4} N^{10}+6447090 k^{4} N^{9} \\
& +2294196 k^{4} N^{8}-33065497 k^{4} N^{7}-67129404 k^{4} N^{6}-41813680 k^{4} N^{5}+13265224 k^{4} N^{4} \\
& +31384928 k^{4} N^{3}+16477280 k^{4} N^{2}+3503808 k^{4} N+195840 k^{4}+7640 k^{3} N^{12} \\
& +939938 k^{3} N^{11}+5751067 k^{3} N^{10}+7728037 k^{3} N^{9}-20304955 k^{3} N^{8}-77635215 k^{3} N^{7} \\
& -101155164 k^{3} N^{6}-58732732 k^{3} N^{5}-5633280 k^{3} N^{4}+10914208 k^{3} N^{3}+5252608 k^{3} N^{2} \\
& +708480 k^{3} N+1004 k^{2} N^{13}+342128 k^{2} N^{12}+2831143 k^{2} N^{11}+6441570 k^{2} N^{10} \\
& -4505918 k^{2} N^{9}-44856020 k^{2} N^{8}-86247029 k^{2} N^{7}-81411198 k^{2} N^{6}-38586456 k^{2} N^{5} \\
& -5465256 k^{2} N^{4}+2291152 k^{2} N^{3}+732672 k^{2} N^{2}+52808 k N^{13}+690048 k N^{12} \\
& +2383148 k N^{11}+985116 k N^{10}-12130796 k N^{9}-33522668 k N^{8}-42209368 k N^{7} \\
& -28755104 k N^{6}-9874528 k N^{5}-1005632 k N^{4}+170496 k N^{3}+57804 N^{13}+332760 N^{12} \\
& +475944 N^{11}-1055904 N^{10}-4895412 N^{9}-7985016 N^{8}-6966624 N^{7}-3346848 N^{6} \\
& \left.\left.-782016 N^{5}-55296 N^{4}\right)\right] /\left[(N-2)(N+1)(N+2)^{2}(k+N+1)(2 k+N)(2 k+3 N+2)\right. \\
& \times d(N, k) p(N, k)], \\
& a_{47}=\left[1 2 ( k + N ) \left(188 k^{4} N^{6}+872 k^{4} N^{5}-1196 k^{4} N^{4}-7000 k^{4} N^{3}+960 k^{4} N^{2}+10400 k^{4} N\right.\right. \\
& +1920 k^{4}+654 k^{3} N^{7}+2754 k^{3} N^{6}-4286 k^{3} N^{5}-23506 k^{3} N^{4}+2440 k^{3} N^{3}+45640 k^{3} N^{2} \\
& +25120 k^{3} N+3840 k^{3}+657 k^{2} N^{8}+2938 k^{2} N^{7}-4231 k^{2} N^{6}-26996 k^{2} N^{5}-916 k^{2} N^{4} \\
& +63288 k^{2} N^{3}+56240 k^{2} N^{2}+17440 k^{2} N+1920 k^{2}+202 k N^{9}+1137 k N^{8}-1446 k N^{7} \\
& -12233 k N^{6}-2130 k N^{5}+35970 k N^{4}+43848 k N^{3}+18920 k N^{2}+2720 k N+102 N^{9} \\
& \left.\left.-120 N^{8}-1830 N^{7}-636 N^{6}+7308 N^{5}+11376 N^{4}+6000 N^{3}+960 N^{2}\right)\right] /[(N-2) \\
& \left.\times(N+2)^{2}(2 k+N)(2 k+3 N+2) p(N, k)\right], \\
& a_{48}=\left[6 ( N + 1 ) ( N + 2 ) \left(400 k^{7} N^{3}-1440 k^{7} N^{2}-4720 k^{7} N+16320 k^{7}+3150 k^{6} N^{4}\right.\right. \\
& -10140 k^{6} N^{3}-41490 k^{6} N^{2}+114360 k^{6} N+48960 k^{6}+9605 k^{5} N^{5}-28416 k^{5} N^{4} \\
& -155131 k^{5} N^{3}+347094 k^{5} N^{2}+315884 k^{5} N+23280 k^{5}+14110 k^{4} N^{6}-38456 k^{4} N^{5} \\
& -311476 k^{4} N^{4}+562184 k^{4} N^{3}+851094 k^{4} N^{2}+175680 k^{4} N-60720 k^{4}+9980 k^{3} N^{7} \\
& -22836 k^{3} N^{6}-359779 k^{3} N^{5}+505816 k^{3} N^{4}+1173921 k^{3} N^{3}+462242 k^{3} N^{2}-132340 k^{3} N \\
& -77040 k^{3}+2760 k^{2} N^{8}-1936 k^{2} N^{7}-238178 k^{2} N^{6}+255502 k^{2} N^{5}+841998 k^{2} N^{4} \\
& +506154 k^{2} N^{3}-31084 k^{2} N^{2}-128304 k^{2} N-25680 k^{2}+2160 k N^{8}-83652 k N^{7} \\
& +81080 k N^{6}+287596 k N^{5}+203488 k N^{4}+67040 k N^{3}-18432 k N^{2}-17088 k N \\
& \left.\left.-12600 N^{8}+18264 N^{7}+37512 N^{6}+7176 N^{5}+22416 N^{4}+32832 N^{3}+10944 N^{2}\right)\right] / \\
& \times[(N-2)(2 k+N)(2 k+3 N+2) d(N, k) p(N, k)], \\
& a_{49}=\left[-6\left(880 k^{8} N^{5}+23472 k^{8} N^{4}-80848 k^{8} N^{3}-124080 k^{8} N^{2}+181920 k^{8} N+48960 k^{8}\right.\right. \\
& +6088 k^{7} N^{6}+162880 k^{7} N^{5}-599264 k^{7} N^{4}-1150160 k^{7} N^{3}+1245208 k^{7} N^{2}+1143952 k^{7} N \\
& +195840 k^{7}+16266 k^{6} N^{7}+456126 k^{6} N^{6}-1941482 k^{6} N^{5}-4458174 k^{6} N^{4}+3679432 k^{6} N^{3} \\
& +6971784 k^{6} N^{2}+2776480 k^{6} N+293760 k^{6}+21065 k^{5} N^{8}+658199 k^{5} N^{7}-3657717 k^{5} N^{6} \\
& -9599335 k^{5} N^{5}+6131424 k^{5} N^{4}+20594612 k^{5} N^{3}+13282768 k^{5} N^{2}+3110304 k^{5} N \\
& +195840 k^{5}+13595 k^{4} N^{9}+512546 k^{4} N^{8}-4423504 k^{4} N^{7}-12800418 k^{4} N^{6}+6061833 k^{4} N^{5} \\
& +34745808 k^{4} N^{4}+31310524 k^{4} N^{3}+11379328 k^{4} N^{2}+1557376 k^{4} N+48960 k^{4} \\
& +4032 k^{3} N^{10}+201162 k^{3} N^{9}-3500243 k^{3} N^{8}-10995929 k^{3} N^{7}+3165031 k^{3} N^{6} \\
& +35051735 k^{3} N^{5}+40792120 k^{3} N^{4}+19883084 k^{3} N^{3}+4113432 k^{3} N^{2}+261520 k^{3} N
\end{aligned}
$$




$$
\begin{aligned}
& +444 k^{2} N^{11}+29176 k^{2} N^{10}-1738853 k^{2} N^{9}-5977480 k^{2} N^{8}+353060 k^{2} N^{7} \\
& +20792514 k^{2} N^{6}+29840905 k^{2} N^{5}+17942726 k^{2} N^{4}+4764956 k^{2} N^{3}+414376 k^{2} N^{2} \\
& -984 k N^{11}-485480 k N^{10}-1869108 k N^{9}-377396 k N^{8}+6674980 k N^{7}+11423548 k N^{6} \\
& +7942360 k N^{5}+2417904 k N^{4}+231328 k N^{3}-57828 N^{11}-255024 N^{10}-114288 N^{9} \\
& \left.\left.+906360 N^{8}+1771428 N^{7}+1322328 N^{6}+401904 N^{5}+27552 N^{4}\right)\right] /[(N-2)(k+N+1) \\
& \times(2 k+N)(2 k+3 N+2) d(N, k) p(N, k)], \\
& a_{50}=\left[-12(N+1)\left(150 k^{8} N^{4}-240 k^{8} N^{3}-2850 k^{8} N^{2}+2580 k^{8} N+12240 k^{8}+1249 k^{7} N^{5}\right.\right. \\
& -718 k^{7} N^{4}-26483 k^{7} N^{3}+1600 k^{7} N^{2}+102676 k^{7} N+48960 k^{7}+4343 k^{6} N^{6}+3823 k^{6} N^{5} \\
& -104259 k^{6} N^{4}-88667 k^{6} N^{3}+351244 k^{6} N^{2}+415372 k^{6} N+124800 k^{6}+8145 k^{5} N^{7} \\
& +23742 k^{5} N^{6}-219920 k^{5} N^{5}-421350 k^{5} N^{4}+562947 k^{5} N^{3}+1436832 k^{5} N^{2}+892052 k^{5} N \\
& +254400 k^{5}+8745 k^{4} N^{8}+51233 k^{4} N^{7}-262388 k^{4} N^{6}-876902 k^{4} N^{5}+286289 k^{4} N^{4} \\
& +2581741 k^{4} N^{3}+2482290 k^{4} N^{2}+1235264 k^{4} N+320400 k^{4}+5096 k^{3} N^{9}+55166 k^{3} N^{8} \\
& -171993 k^{3} N^{7}-971946 k^{3} N^{6}-325141 k^{3} N^{5}+2578794 k^{3} N^{4}+3459476 k^{3} N^{3} \\
& +2191720 k^{3} N^{2}+992136 k^{3} N+205440 k^{3}+1252 k^{2} N^{10}+29716 k^{2} N^{9}-54803 k^{2} N^{8} \\
& -579053 k^{2} N^{7}-512091 k^{2} N^{6}+1442035 k^{2} N^{5}+2571930 k^{2} N^{4}+1703922 k^{2} N^{3} \\
& +887596 k^{2} N^{2}+367824 k^{2} N+51360 k^{2}+6328 k N^{10}-6856 k N^{9}-167380 k N^{8} \\
& -232872 k N^{7}+441324 k N^{6}+992072 k N^{5}+523128 k N^{4}+83120 k N^{3}+66240 k N^{2} \\
& +34176 k N-924 N^{10}-17076 N^{9}-30156 N^{8}+66300 N^{7}+163656 N^{6}+32904 N^{5} \\
& \left.\left.-127056 N^{4}-98496 N^{3}-21888 N^{2}\right)\right] /[(N-2)(k+N+1)(2 k+N)(2 k+3 N+2) \\
& \times d(N, k) p(N, k)], \\
& a_{51}=\left[-12(N+1)(N+2)\left(20 k^{4} N^{2}-140 k^{4} N+240 k^{4}+80 k^{3} N^{3}-520 k^{3} N^{2}+680 k^{3} N\right.\right. \\
& +480 k^{3}+97 k^{2} N^{4}-663 k^{2} N^{3}+660 k^{2} N^{2}+1160 k^{2} N+240 k^{2}+34 k N^{5}-349 k N^{4} \\
& \left.\left.+297 k N^{3}+920 k N^{2}+340 k N-66 N^{5}+54 N^{4}+240 N^{3}+120 N^{2}\right)\right] /[(N-2)(2 k+N) \\
& \times(2 k+3 N+2) p(N, k)], \\
& a_{52}=\left[1 2 ( N + 1 ) ( N + 2 ) \left(150 k^{6} N^{3}-540 k^{6} N^{2}-1770 k^{6} N+6120 k^{6}+935 k^{5} N^{4}-2430 k^{5} N^{3}\right.\right. \\
& -14705 k^{5} N^{2}+34620 k^{5} N+18360 k^{5}+2120 k^{4} N^{5}-2762 k^{4} N^{4}-43742 k^{4} N^{3} \\
& +87242 k^{4} N^{2}+53262 k^{4} N+18360 k^{4}+2060 k^{3} N^{6}+1608 k^{3} N^{5}-59061 k^{3} N^{4} \\
& +129870 k^{3} N^{3}+47143 k^{3} N^{2}-4416 k^{3} N+6120 k^{3}+720 k^{2} N^{7}+4448 k^{2} N^{6}-37640 k^{2} N^{5} \\
& +124048 k^{2} N^{4}+10136 k^{2} N^{3}-102168 k^{2} N^{2}-21288 k^{2} N+1968 k N^{7}-10908 k N^{6} \\
& +69624 k N^{5}+612 k N^{4}-115392 k N^{3}-47904 k N^{2}-1152 N^{7}+16704 N^{6}+1152 N^{5} \\
& \left.\left.-36000 N^{4}-19296 N^{3}\right)\right] /[(N-2)(2 k+N)(2 k+3 N+2) d(N, k) p(N, k)],
\end{aligned}
$$

where

$$
\begin{aligned}
& d(N, k) \equiv 5 k^{2} N+17 k^{2}+10 k N^{2}+39 k N+17 k+22 N^{2}+22 N, \\
& p(N, k) \equiv 7 k^{2} N+107 k^{2}+14 k N^{2}+221 k N+107 k+114 N^{2}+114 N .
\end{aligned}
$$

Although each coefficient function is rather complicated, as before, the exact (or large $N$ limit) expressions for the zero mode eigenvalue equations are rather simple. One of the reasons why (D.1) is so complicated comes from the simplification for the independent terms. Originally, after collecting the results from appendices $B$ and $C$, there are 
approximately one hundred fifty terms. By rewriting the dependent terms in terms of the above 52 terms using Mathematica, rather simple coefficient functions can combine and become quite complicated. In particular, the coefficient functions appearing in the derivative terms in spin-5 current are most complicated.

Furthermore, the large $N$ 't Hooft limit (4.1) on these coefficient functions leads to

$$
\begin{aligned}
& a_{1} \rightarrow \frac{6(\lambda-2)^{2}(\lambda-1) N}{\lambda^{3}(\lambda+2)}, \\
& a_{4} \rightarrow \frac{12\left(\lambda^{2}-6 \lambda+10\right) N}{(\lambda-2) \lambda(\lambda+2)}, \\
& a_{7} \rightarrow \frac{12(\lambda-6) N}{(\lambda-2)(\lambda+2)}, \\
& a_{10} \rightarrow-\frac{6 \lambda N}{\lambda+2}, \\
& a_{13} \rightarrow \frac{96(\lambda-1)\left(4 \lambda^{2}-29\right) N}{7 \lambda^{3}(\lambda+1)(\lambda+2)}, \\
& a_{16} \rightarrow-\frac{96\left(4 \lambda^{2}-29\right)}{7 \lambda(\lambda+1)(\lambda+2)}, \\
& a_{18} \rightarrow \frac{288\left(4 \lambda^{2}-29\right) N}{7(\lambda-2) \lambda(\lambda+1)(\lambda+2)}, \\
& a_{20} \rightarrow-\frac{96\left(4 \lambda^{2}-29\right)}{7(\lambda-2)(\lambda+1)(\lambda+2)}, \\
& a_{22} \rightarrow \frac{24 \lambda\left(7 \lambda^{3}-34 \lambda^{2}-7 \lambda+334\right)}{7(\lambda-2)(\lambda-1)(\lambda+1)(\lambda+2)}, \\
& a_{24} \rightarrow \frac{96 \lambda^{2}\left(4 \lambda^{2}-29\right)}{7(\lambda-2)(\lambda-1)(\lambda+1)(\lambda+2) N}, \\
& a_{26} \rightarrow-\frac{6\left(9 \lambda^{2}-40 \lambda+4\right) N^{2}}{5 \lambda^{2}(\lambda+2)}, \\
& a_{28} \rightarrow \frac{207(\lambda-2) N^{2}}{5 \lambda^{2}}, \\
& a_{31} \rightarrow-\frac{6\left(3 \lambda^{2}-4 \lambda+8\right) N^{2}}{\lambda(\lambda+2)}, \\
& a_{33} \rightarrow-\frac{18\left(\lambda^{2}-4 \lambda+8\right) N^{2}}{\lambda(\lambda+2)}, \\
& a_{35} \rightarrow \frac{6\left(\lambda^{3}-6 \lambda^{2}-4 \lambda+40\right) N^{2}}{(\lambda-2) \lambda(\lambda+2)}, \\
& a_{37} \rightarrow \frac{3\left(3 \lambda^{2}+30 \lambda-112\right) N^{2}}{5(\lambda-2)(\lambda+2)}, \\
& a_{39} \rightarrow \frac{6\left(9 \lambda^{2}+20 \lambda-56\right) N^{2}}{5(\lambda-2)(\lambda+2)}, \\
& a_{42} \rightarrow-\frac{48(\lambda-1)\left(2 \lambda^{2}-11\right) N^{3}}{7 \lambda^{3}(\lambda+2)}, \\
& a_{44} \rightarrow \frac{60(\lambda-4)(2 \lambda+1) N^{3}}{7 \lambda^{2}(\lambda+2)}, \\
& a_{46} \rightarrow-\frac{6\left(70 \lambda^{3}-113 \lambda^{2}+420 \lambda-628\right) N^{3}}{35 \lambda^{2}(\lambda+2)}, \\
& a_{48} \rightarrow-\frac{6\left(7 \lambda^{3}-19 \lambda^{2}+70 \lambda+80\right) N^{3}}{7(\lambda-2) \lambda(\lambda+2)},
\end{aligned}
$$

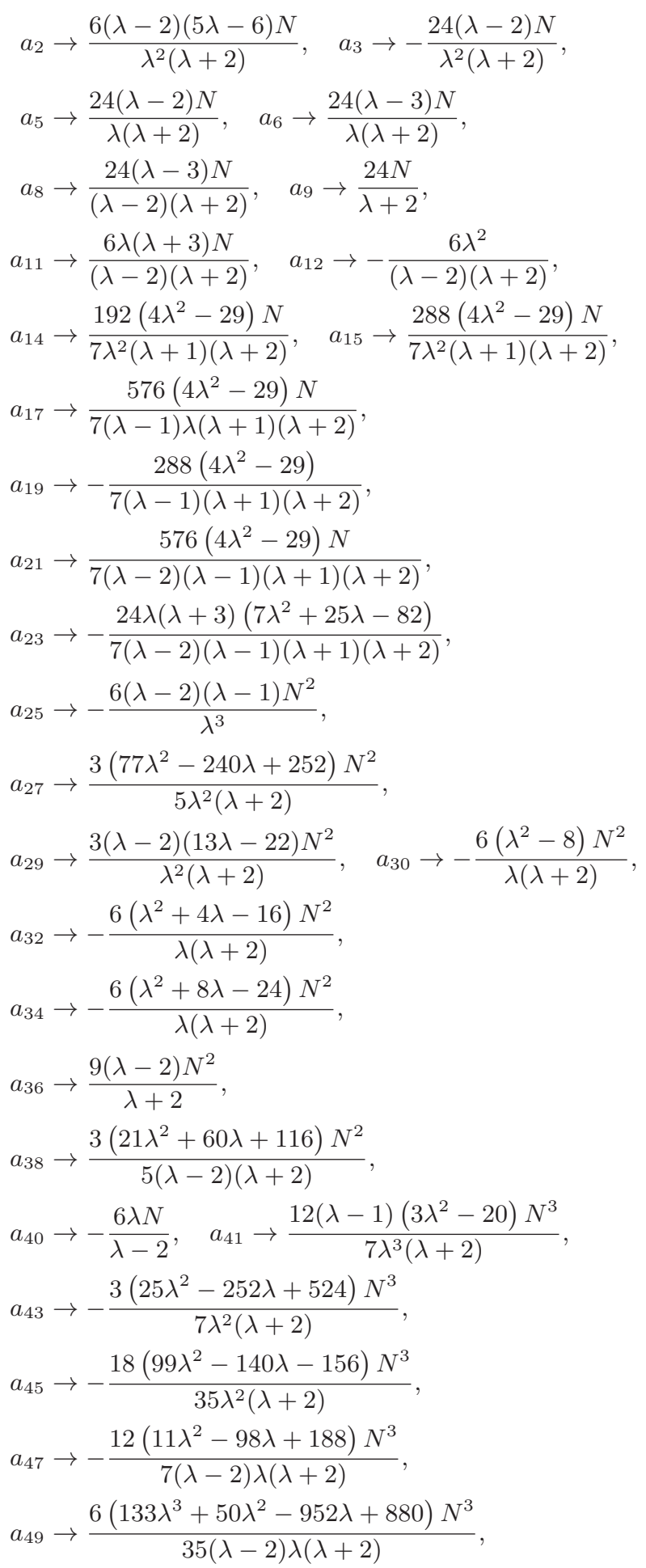




$$
\begin{aligned}
& a_{50} \rightarrow \frac{12\left(14 \lambda^{3}+100 \lambda^{2}+49 \lambda+150\right) N^{3}}{35(\lambda-2) \lambda(\lambda+2)}, \quad a_{51} \rightarrow-\frac{12\left(3 \lambda^{2}-20\right) N^{2}}{7(\lambda-2)(\lambda+2)}, \\
& a_{52} \rightarrow \frac{12(\lambda-10)(\lambda+3) N^{2}}{7(\lambda-2)(\lambda+2)} .
\end{aligned}
$$

In this case, each coefficient function has a very simple form after taking the large $N$ limit. The large $N$ behavior of these coefficient functions can be classified as follows:

$$
\begin{aligned}
a_{24} & \rightarrow \frac{1}{N}, \\
a_{12}, a_{16}, a_{19}, a_{20}, a_{22}, a_{23}, & \rightarrow \text { const, }
\end{aligned}
$$

$a_{1}, a_{2}, a_{3}, a_{4}, a_{5}, a_{6}, a_{7}, a_{8}, a_{9}, a_{10}, a_{11}, a_{13}, a_{14}, a_{15}, a_{17}, a_{18}, a_{21}, a_{40} \rightarrow N$, $a_{25}, a_{26}, a_{27}, a_{28}, a_{29}, a_{30}, a_{31}, a_{32}, a_{33}, a_{34}, a_{35}, a_{36}, a_{37}, a_{38}, a_{39}, a_{51}, a_{52} \rightarrow N^{2}$,

$$
a_{41}, a_{42}, a_{43}, a_{44}, a_{45}, a_{46}, a_{47}, a_{48}, a_{49}, a_{50} \rightarrow N^{3} \text {. }
$$

In section 4, the eigenvalue equations and three-point functions are analyzed using (D.3) and (D.4).

\section{E Eigenvalue equations for the spin-5 current on the perturbative state}

Appendix $E$ presents the zero mode eigenvalue equations on the 52 terms of spin- 5 current with perturbative state $|(f ; 0)\rangle$. At each expression, the exact $N$-dependent expressions appearing in (4.10)-(4.15) are assumed. The only large $N$ limit is presented.

$$
\begin{aligned}
d^{a b f} d^{f c g} d^{g d e}\left(J^{a} J^{b} J^{c} J^{d} J^{e}\right)_{0}|(f ; 0)\rangle & =-\frac{1}{N} d^{a b f} d^{f c g} d^{g d e} \operatorname{Tr}\left(T^{a} T^{b} T^{c} T^{d} T^{e}\right)|(f ; 0)\rangle \\
& \rightarrow i N^{4}|(f ; 0)\rangle, \\
d^{a b f} d^{f c g} d^{g d e}\left(J^{a} J^{b} J^{c} J^{d} K^{e}\right)_{0}|(f ; 0)\rangle & =\frac{1}{N} d^{a b f} d^{f c g} d^{g d e} \operatorname{Tr}\left(T^{a} T^{b} T^{c} T^{d} T^{e}\right)|(f ; 0)\rangle \\
& \rightarrow-i N^{4}|(f ; 0)\rangle, \\
d^{a b f} d^{f e g} d^{g c d}\left(J^{a} J^{b} J^{c} J^{d} K^{e}\right)_{0}|(f ; 0)\rangle & =\frac{1}{N} d^{a b f} d^{f c g} d^{g d e} \operatorname{Tr}\left(T^{a} T^{b} T^{c} T^{d} T^{e}\right)|(f ; 0)\rangle \\
& \rightarrow-i N^{4}|(f ; 0)\rangle, \\
d^{a b f} d^{f c g} d^{g d e}\left(J^{a} J^{b} J^{c} K^{d} K^{e}\right)_{0}|(f ; 0)\rangle & =-\frac{1}{N} d^{a b f} d^{f c g} d^{g d e} \operatorname{Tr}\left(T^{a} T^{b} T^{c} T^{d} T^{e}\right)|(f ; 0)\rangle \\
& \rightarrow i N^{4}|(f ; 0)\rangle, \\
d^{b d f} d^{f a g} d^{g c e}\left(J^{a} J^{b} J^{c} K^{d} K^{e}\right)_{0}|(f ; 0)\rangle & =-\frac{1}{N} d^{a c f} d^{f b g} d^{g d e} \operatorname{Tr}\left(T^{a} T^{b} T^{c} T^{d} T^{e}\right)|(f ; 0)\rangle \\
& \rightarrow-4 i N^{2}|(f ; 0)\rangle, \\
d^{a b f} d^{f d g} d^{g c e}\left(J^{a} J^{b} J^{c} K^{d} K^{e}\right)_{0}|(f ; 0)\rangle & =-\frac{1}{N} d^{a b f} d^{f c g} d^{g d e} \operatorname{Tr}\left(T^{a} T^{b} T^{c} T^{d} T^{e}\right)|(f ; 0)\rangle \\
& \rightarrow i N^{4}|(f ; 0)\rangle, \\
d^{a b f} d^{f c g} d^{g d e}\left(J^{a} J^{b} K^{c} K^{d} K^{e}\right)_{0}|(f ; 0)\rangle & =\frac{1}{N} d^{a b f} d^{f c g} d^{g d e} \operatorname{Tr}\left(T^{a} T^{b} T^{c} T^{d} T^{e}\right)|(f ; 0)\rangle \\
& \rightarrow-i N^{4}|(f ; 0)\rangle, \\
d^{b c f} d^{f a g} d^{g d e}\left(J^{a} J^{b} K^{c} K^{d} K^{e}\right)_{0}|(f ; 0)\rangle & =\frac{1}{N} d^{a c f} d^{f b g} d^{g d e} \operatorname{Tr}\left(T^{a} T^{b} T^{c} T^{d} T^{e}\right)|(f ; 0)\rangle
\end{aligned}
$$




$$
\begin{aligned}
& \rightarrow 4 i N^{2}|(f ; 0)\rangle \\
& d^{a c f} d^{f d g} d^{g b e}\left(J^{a} J^{b} K^{c} K^{d} K^{e}\right)_{0}|(f ; 0)\rangle=\frac{1}{N} d^{a b f} d^{f c g} d^{g d e} \operatorname{Tr}\left(T^{a} T^{b} T^{c} T^{d} T^{e}\right)|(f ; 0)\rangle \\
& \rightarrow-i N^{4}|(f ; 0)\rangle, \\
& d^{a b f} d^{f c g} d^{g d e}\left(J^{a} K^{b} K^{c} K^{d} K^{e}\right)_{0}|(f ; 0)\rangle=-\frac{1}{N} d^{a b f} d^{f c g} d^{g d e} \operatorname{Tr}\left(T^{a} T^{b} T^{c} T^{d} T^{e}\right)|(f ; 0)\rangle \\
& \rightarrow i N^{4}|(f ; 0)\rangle, \\
& d^{b c f} d^{f a g} d^{g d e}\left(J^{a} K^{b} K^{c} K^{d} K^{e}\right)_{0}|(f ; 0)\rangle=-\frac{1}{N} d^{a b f} d^{f c g} d^{g d e} \operatorname{Tr}\left(T^{a} T^{b} T^{c} T^{d} T^{e}\right)|(f ; 0)\rangle \\
& \rightarrow i N^{4}|(f ; 0)\rangle, \\
& d^{a b f} d^{f c g} d^{g d e}\left(K^{a} K^{b} K^{c} K^{d} K^{e}\right)_{0}|(f ; 0)\rangle=\frac{1}{N} d^{a b f} d^{f c g} d^{g d e} \operatorname{Tr}\left(T^{a} T^{b} T^{c} T^{d} T^{e}\right)|(f ; 0)\rangle \\
& \rightarrow-i N^{4}|(f ; 0)\rangle \text {, } \\
& d^{a b c} \delta^{d e}\left(J^{a} J^{b} J^{c} J^{d} J^{e}\right)_{0}|(f ; 0)\rangle=-\frac{1}{N} d^{a b c} \delta^{d e} \operatorname{Tr}\left(T^{a} T^{b} T^{c} T^{d} T^{e}\right)|(f ; 0)\rangle \\
& \rightarrow i N^{3}|(f ; 0)\rangle, \\
& d^{a b c} \delta^{d e}\left(J^{a} J^{b} J^{c} J^{d} K^{e}\right)_{0}|(f ; 0)\rangle=\frac{1}{N} d^{a b c} \delta^{d e} \operatorname{Tr}\left(T^{a} T^{b} T^{c} T^{d} T^{e}\right)|(f ; 0)\rangle \\
& \rightarrow-i N^{3}|(f ; 0)\rangle \text {, } \\
& d^{a b e} \delta^{c d}\left(J^{a} J^{b} J^{c} J^{d} K^{e}\right)_{0}|(f ; 0)\rangle=\frac{1}{N} d^{a b c} \delta^{d e} \operatorname{Tr}\left(T^{a} T^{b} T^{c} T^{d} T^{e}\right)|(f ; 0)\rangle \\
& \rightarrow-i N^{3}|(f ; 0)\rangle, \\
& d^{a b c} \delta^{d e}\left(J^{a} J^{b} J^{c} K^{d} K^{e}\right)_{0}|(f ; 0)\rangle=-\frac{1}{N} d^{a b c} \delta^{d e} \operatorname{Tr}\left(T^{a} T^{b} T^{c} T^{d} T^{e}\right)|(f ; 0)\rangle \\
& \rightarrow i N^{3}|(f ; 0)\rangle \text {, } \\
& d^{a b d} \delta^{c e}\left(J^{a} J^{b} J^{c} K^{d} K^{e}\right)_{0}|(f ; 0)\rangle=-\frac{1}{N} d^{a b c} \delta^{d e} \operatorname{Tr}\left(T^{a} T^{b} T^{c} T^{d} T^{e}\right)|(f ; 0)\rangle \\
& \rightarrow i N^{3}|(f ; 0)\rangle \text {, } \\
& \delta^{a b} d^{c d e}\left(J^{a} J^{b} J^{c} K^{d} K^{e}\right)_{0}|(f ; 0)\rangle=-\frac{1}{N} d^{a b c} \delta^{d e} \operatorname{Tr}\left(T^{a} T^{b} T^{c} T^{d} T^{e}\right)|(f ; 0)\rangle \\
& \rightarrow i N^{3}|(f ; 0)\rangle, \\
& d^{a b c} \delta^{d e}\left(J^{a} J^{b} K^{c} K^{d} K^{e}\right)_{0}|(f ; 0)\rangle=\frac{1}{N} d^{a b c} \delta^{d e} \operatorname{Tr}\left(T^{a} T^{b} T^{c} T^{d} T^{e}\right)|(f ; 0)\rangle \\
& \rightarrow-i N^{3}|(f ; 0)\rangle \text {, } \\
& \delta^{a b} d^{c d e}\left(J^{a} J^{b} K^{c} K^{d} K^{e}\right)_{0}|(f ; 0)\rangle=\frac{1}{N} d^{a b c} \delta^{d e} \operatorname{Tr}\left(T^{a} T^{b} T^{c} T^{d} T^{e}\right)|(f ; 0)\rangle \\
& \rightarrow-i N^{3}|(f ; 0)\rangle, \\
& \delta^{a c} d^{b d e}\left(J^{a} J^{b} K^{c} K^{d} K^{e}\right)_{0}|(f ; 0)\rangle=\frac{1}{N} d^{a b c} \delta^{d e} \operatorname{Tr}\left(T^{a} T^{b} T^{c} T^{d} T^{e}\right)|(f ; 0)\rangle \\
& \rightarrow-i N^{3}|(f ; 0)\rangle, \\
& d^{a b c} \delta^{d e}\left(J^{a} K^{b} K^{c} K^{d} K^{e}\right)_{0}|(f ; 0)\rangle=-\frac{1}{N} d^{a b c} \delta^{d e} \operatorname{Tr}\left(T^{a} T^{b} T^{c} T^{d} T^{e}\right)|(f ; 0)\rangle \\
& \rightarrow i N^{3}|(f ; 0)\rangle \text {, } \\
& \delta^{a b} d^{c d e}\left(J^{a} K^{b} K^{c} K^{d} K^{e}\right)_{0}|(f ; 0)\rangle=-\frac{1}{N} d^{a b c} \delta^{d e} \operatorname{Tr}\left(T^{a} T^{b} T^{c} T^{d} T^{e}\right)|(f ; 0)\rangle
\end{aligned}
$$




$$
\begin{aligned}
& \rightarrow i N^{3}|(f ; 0)\rangle, \\
& d^{a b c} \delta^{d e}\left(K^{a} K^{b} K^{c} K^{d} K^{e}\right)_{0}|(f ; 0)\rangle=\frac{1}{N} d^{a b c} \delta^{d e} \operatorname{Tr}\left(T^{a} T^{b} T^{c} T^{d} T^{e}\right)|(f ; 0)\rangle \\
& \rightarrow-i N^{3}|(f ; 0)\rangle \text {, } \\
& f^{a d e} d^{b c e}\left(J^{a} J^{b} J^{c} \partial J^{d}\right)_{0}|(f ; 0)\rangle=\frac{1}{N} d^{a b e} f^{c d e} \operatorname{Tr}\left(T^{a} T^{b} T^{c} T^{d}\right)|(f ; 0)\rangle \\
& \rightarrow i N^{3}|(f ; 0)\rangle \text {, } \\
& d^{a b e} f^{c d e}\left(J^{a} J^{b} J^{c} \partial K^{d}\right)_{0}|(f ; 0)\rangle=\frac{1}{N} d^{a b e} f^{c d e} \operatorname{Tr}\left(T^{a} T^{b} T^{c} T^{d}\right)|(f ; 0)\rangle \\
& \rightarrow i N^{3}|(f ; 0)\rangle, \\
& d^{a b e} f^{c d e}\left(J^{a} J^{b} \partial J^{c} K^{d}\right)_{0}|(f ; 0)\rangle=\frac{1}{N} d^{a b e} f^{c d e} \operatorname{Tr}\left(T^{a} T^{b} T^{c} T^{d}\right)|(f ; 0)\rangle \\
& \rightarrow i N^{3}|(f ; 0)\rangle \text {, } \\
& d^{a c e} f^{b d e}\left(J^{a} J^{b} \partial J^{c} K^{d}\right)_{0}|(f ; 0)\rangle=\frac{1}{N} d^{a c e} f^{b d e} \operatorname{Tr}\left(T^{a} T^{b} T^{c} T^{d}\right)|(f ; 0)\rangle \\
& =0 \text {, } \\
& d^{a d e} f^{b c e}\left(J^{a} J^{b} \partial J^{c} K^{d}\right)_{0}|(f ; 0)\rangle=\frac{1}{N} d^{a b e} f^{c d e} \operatorname{Tr}\left(T^{a} T^{b} T^{c} T^{d}\right)|(f ; 0)\rangle \\
& \rightarrow i N^{3}|(f ; 0)\rangle \text {, } \\
& d^{a b e} f^{c d e}\left(J^{a} J^{b} K^{c} \partial K^{d}\right)_{0}|(f ; 0)\rangle=\frac{1}{N} d^{a b e} f^{c d e} \operatorname{Tr}\left(T^{a} T^{b} T^{c} T^{d}\right)|(f ; 0)\rangle \\
& \rightarrow i N^{3}|(f ; 0)\rangle \text {, } \\
& d^{a c e} f^{b d e}\left(J^{a} J^{b} K^{c} \partial K^{d}\right)_{0}|(f ; 0)\rangle=-\frac{1}{N} d^{a b e} f^{c d e} \operatorname{Tr}\left(T^{a} T^{b} T^{c} T^{d}\right)|(f ; 0)\rangle \\
& \rightarrow-i N^{3}|(f ; 0)\rangle, \\
& d^{a d e} f^{b c e}\left(J^{a} J^{b} K^{c} \partial K^{d}\right)_{0}|(f ; 0)\rangle=\frac{1}{N} d^{a c e} f^{b d e} \operatorname{Tr}\left(T^{a} T^{b} T^{c} T^{d}\right)|(f ; 0)\rangle \\
& =0 \text {, } \\
& d^{a c e} f^{b d e}\left(J^{a} \partial J^{b} K^{c} K^{d}\right)_{0}|(f ; 0)\rangle=-\frac{1}{N} d^{a b e} f^{c d e} \operatorname{Tr}\left(T^{a} T^{b} T^{c} T^{d}\right)|(f ; 0)\rangle \\
& \rightarrow-i N^{3}|(f ; 0)\rangle \text {, } \\
& f^{a d e} d^{b c e}\left(J^{a} \partial J^{b} K^{c} K^{d}\right)_{0}|(f ; 0)\rangle=-\frac{1}{N} d^{a c e} f^{b d e} \operatorname{Tr}\left(T^{a} T^{b} T^{c} T^{d}\right)|(f ; 0)\rangle \\
& =0 \text {, } \\
& f^{a b e} d^{c d e}\left(J^{a} \partial J^{b} K^{c} K^{d}\right)_{0}|(f ; 0)\rangle=-\frac{1}{N} d^{a b e} f^{c d e} \operatorname{Tr}\left(T^{a} T^{b} T^{c} T^{d}\right)|(f ; 0)\rangle \\
& \rightarrow-i N^{3}|(f ; 0)\rangle, \\
& d^{a b e} f^{c d e}\left(J^{a} K^{b} K^{c} \partial K^{d}\right)_{0}|(f ; 0)\rangle=-\frac{1}{N} d^{a b e} f^{c d e} \operatorname{Tr}\left(T^{a} T^{b} T^{c} T^{d}\right)|(f ; 0)\rangle \\
& \rightarrow-i N^{3}|(f ; 0)\rangle \text {, } \\
& f^{a d e} d^{b c e}\left(J^{a} K^{b} K^{c} \partial K^{d}\right)_{0}|(f ; 0)\rangle=\frac{1}{N} d^{a b e} f^{c d e} \operatorname{Tr}\left(T^{a} T^{b} T^{c} T^{d}\right)|(f ; 0)\rangle \\
& \rightarrow i N^{3}|(f ; 0)\rangle \text {, } \\
& f^{a c e} d^{b d e}\left(J^{a} K^{b} K^{c} \partial K^{d}\right)_{0}|(f ; 0)\rangle=-\frac{1}{N} d^{a c e} f^{b d e} \operatorname{Tr}\left(T^{a} T^{b} T^{c} T^{d}\right)|(f ; 0)\rangle
\end{aligned}
$$




$$
\begin{aligned}
& =0 \text {, } \\
& f^{a d e} d^{b c e}\left(\partial J^{a} K^{b} K^{c} K^{d}\right)_{0}|(f ; 0)\rangle=\frac{1}{N} d^{a b e} f^{c d e} \operatorname{Tr}\left(T^{a} T^{b} T^{c} T^{d}\right)|(f ; 0)\rangle \\
& \rightarrow i N^{3}|(f ; 0)\rangle \text {, } \\
& f^{a d e} d^{b c e}\left(K^{a} K^{b} K^{c} \partial K^{d}\right)_{0}|(f ; 0)\rangle=-\frac{1}{N} d^{a b e} f^{c d e} \operatorname{Tr}\left(T^{a} T^{b} T^{c} T^{d}\right)|(f ; 0)\rangle \\
& \rightarrow-i N^{3}|(f ; 0)\rangle, \\
& d^{a b c}\left(J^{a} J^{b} \partial^{2} J^{c}\right)_{0}|(f ; 0)\rangle=-\frac{2}{N} d^{a b c} \operatorname{Tr}\left(T^{a} T^{b} T^{c}\right)|(f ; 0)\rangle \\
& \rightarrow-2 i N^{2}|(f ; 0)\rangle, \\
& d^{a b c}\left(J^{a} \partial J^{b} \partial J^{c}\right)_{0}|(f ; 0)\rangle=-\frac{1}{N} d^{a b c} \operatorname{Tr}\left(T^{a} T^{b} T^{c}\right)|(f ; 0)\rangle \\
& \rightarrow-i N^{2}|(f ; 0)\rangle \\
& d^{a b c}\left(J^{a} \partial^{2} J^{b} K^{c}\right)_{0}|(f ; 0)\rangle=\frac{2}{N} d^{a b c} \operatorname{Tr}\left(T^{a} T^{b} T^{c}\right)|(f ; 0)\rangle \\
& \rightarrow 2 i N^{2}|(f ; 0)\rangle \text {, } \\
& d^{a b c}\left(J^{a} J^{b} \partial^{2} K^{c}\right)_{0}|(f ; 0)\rangle=\frac{2}{N} d^{a b c} \operatorname{Tr}\left(T^{a} T^{b} T^{c}\right)|(f ; 0)\rangle \\
& \rightarrow 2 i N^{2}|(f ; 0)\rangle \text {, } \\
& d^{a b c}\left(\partial J^{a} \partial J^{b} K^{c}\right)_{0}|(f ; 0)\rangle=\frac{1}{N} d^{a b c} \operatorname{Tr}\left(T^{a} T^{b} T^{c}\right)|(f ; 0)\rangle \\
& \rightarrow i N^{2}|(f ; 0)\rangle, \\
& d^{a b c}\left(J^{a} \partial J^{b} \partial K^{c}\right)_{0}|(f ; 0)\rangle=\frac{1}{N} d^{a b c} \operatorname{Tr}\left(T^{a} T^{b} T^{c}\right)|(f ; 0)\rangle \\
& \rightarrow i N^{2}|(f ; 0)\rangle \text {, } \\
& d^{a b c}\left(\partial^{2} J^{a} K^{b} K^{c}\right)_{0}|(f ; 0)\rangle=-\frac{2}{N} d^{a b c} \operatorname{Tr}\left(T^{a} T^{b} T^{c}\right)|(f ; 0)\rangle \\
& \rightarrow-2 i N^{2}|(f ; 0)\rangle, \\
& d^{a b c}\left(J^{a} K^{b} \partial^{2} K^{c}\right)_{0}|(f ; 0)\rangle=-\frac{2}{N} d^{a b c} \operatorname{Tr}\left(T^{a} T^{b} T^{c}\right)|(f ; 0)\rangle \\
& \rightarrow-2 i N^{2}|(f ; 0)\rangle, \\
& d^{a b c}\left(\partial J^{a} K^{b} \partial K^{c}\right)_{0}|(f ; 0)\rangle=-\frac{1}{N} d^{a b c} \operatorname{Tr}\left(T^{a} T^{b} T^{c}\right)|(f ; 0)\rangle \\
& \rightarrow-i N^{2}|(f ; 0)\rangle \text {, } \\
& d^{a b c}\left(J^{a} \partial K^{b} \partial K^{c}\right)_{0}|(f ; 0)\rangle=-\frac{1}{N} d^{a b c} \operatorname{Tr}\left(T^{a} T^{b} T^{c}\right)|(f ; 0)\rangle \\
& \rightarrow-i N^{2}|(f ; 0)\rangle, \\
& d^{a b c}\left(K^{a} K^{b} \partial^{2} K^{c}\right)_{0}|(f ; 0)\rangle=\frac{2}{N} d^{a b c} \operatorname{Tr}\left(T^{a} T^{b} T^{c}\right)|(f ; 0)\rangle \\
& \rightarrow 2 i N^{2}|(f ; 0)\rangle \text {, } \\
& d^{a b c}\left(K^{a} \partial K^{b} \partial K^{c}\right)_{0}|(f ; 0)\rangle=\frac{1}{N} d^{a b c} \operatorname{Tr}\left(T^{a} T^{b} T^{c}\right)|(f ; 0)\rangle \\
& \rightarrow i N^{2}|(f ; 0)\rangle \text {. }
\end{aligned}
$$


From these results, the large $N$ behavior for these 52 eigenvalue equations can be classified as follows:

(5th, 8th, 41st, 42nd, 43rd, 44th, 45th, 46th, 47th, 48th, 49th, 50th, 51st, 52nd) - terms $\rightarrow N^{2}$, (13th, 14th, 15th, 16th, 17th, 18th, 19th, 20th, 21st, 22nd, 23rd, 24th, 25th, 26th, 27th, 29th,

$$
\begin{array}{r}
\text { 30th, 31st, 33rd, 35th, 36th, 37th, 39th, 40th) - terms } \rightarrow N^{3}, \\
\text { (1st, 2nd, 3rd, 4th, 6th, 7th, 9th, 10th, 11th, 12th) - terms } \rightarrow N^{4} .
\end{array}
$$

In section 4, the leading $N^{5}$ behavior in the spin-5 current is analyzed using (D.4) and (E.53).

\section{F Eigenvalue equations for other higher spin currents of spins $s=2,3,4$}

The eigenvalue equations for the zero mode of the higher spin- 4 current can be analyzed as follows:

$$
\begin{aligned}
& d^{a b c d}\left(J^{a} J^{b} J^{c} J^{d}\right)_{0}|(f ; 0)\rangle=\frac{1}{N} d^{a b c d} \operatorname{Tr}\left(T^{a} T^{b} T^{c} T^{d}\right)|(f ; 0)\rangle \\
& =\frac{2\left(N^{2}-1\right)\left(N^{2}-4\right)\left(N^{2}-9\right)}{N\left(N^{2}+1\right)}|(f ; 0)\rangle \rightarrow 2 N^{3}|(f ; 0)\rangle, \\
& d^{a b c d}\left(J^{a} J^{b} J^{c} K^{d}\right)_{0}|(f ; 0)\rangle=-\frac{1}{N} d^{a b c d} \operatorname{Tr}\left(T^{a} T^{b} T^{c} T^{d}\right)|(f ; 0)\rangle \\
& =-\frac{2\left(N^{2}-1\right)\left(N^{2}-4\right)\left(N^{2}-9\right)}{N\left(N^{2}+1\right)}|(f ; 0)\rangle \rightarrow-2 N^{3}|(f ; 0)\rangle, \\
& d^{a b c d}\left(J^{a} J^{b} K^{c} K^{d}\right)_{0}|(f ; 0)\rangle=\frac{1}{N} d^{a b c d} \operatorname{Tr}\left(T^{a} T^{b} T^{c} T^{d}\right)|(f ; 0)\rangle \\
& =\frac{2\left(N^{2}-1\right)\left(N^{2}-4\right)\left(N^{2}-9\right)}{N\left(N^{2}+1\right)}|(f ; 0)\rangle \rightarrow 2 N^{3}|(f ; 0)\rangle, \\
& d^{a b c d}\left(J^{a} K^{b} K^{c} K^{d}\right)_{0}|(f ; 0)\rangle=-\frac{1}{N} d^{a b c d} \operatorname{Tr}\left(T^{a} T^{b} T^{c} T^{d}\right)|(f ; 0)\rangle \\
& =-\frac{2\left(N^{2}-1\right)\left(N^{2}-4\right)\left(N^{2}-9\right)}{N\left(N^{2}+1\right)}|(f ; 0)\rangle \rightarrow-2 N^{3}|(f ; 0)\rangle, \\
& d^{a b c d}\left(K^{a} K^{b} K^{c} K^{d}\right)_{0}|(f ; 0)\rangle=\frac{1}{N} d^{a b c d} \operatorname{Tr}\left(T^{a} T^{b} T^{c} T^{d}\right)|(f ; 0)\rangle \\
& =\frac{2\left(N^{2}-1\right)\left(N^{2}-4\right)\left(N^{2}-9\right)}{N\left(N^{2}+1\right)}|(f ; 0)\rangle \rightarrow 2 N^{3}|(f ; 0)\rangle, \\
& d^{a b e} d^{c d e}\left(J^{a} J^{b} J^{c} J^{d}\right)_{0}|(f ; 0)\rangle=\frac{1}{N} d^{a b e} d^{c d e} \operatorname{Tr}\left(T^{a} T^{b} T^{c} T^{d}\right)|(f ; 0)\rangle \\
& =\frac{1}{N^{3}}\left(N^{2}-4\right)^{2}\left(N^{2}-1\right)|(f ; 0)\rangle \rightarrow N^{3}|(f ; 0)\rangle, \\
& d^{a b e} d^{c d e}\left(J^{a} J^{b} J^{c} K^{d}\right)_{0}|(f ; 0)\rangle=-\frac{1}{N} d^{a b e} d^{c d e} \operatorname{Tr}\left(T^{a} T^{b} T^{c} T^{d}\right)|(f ; 0)\rangle \\
& =-\frac{1}{N^{3}}\left(N^{2}-4\right)^{2}\left(N^{2}-1\right)|(f ; 0)\rangle \rightarrow-N^{3}|(f ; 0)\rangle, \\
& d^{a b e} d^{c d e}\left(J^{a} J^{b} K^{c} K^{d}\right)_{0}|(f ; 0)\rangle=\frac{1}{N} d^{a b e} d^{c d e} \operatorname{Tr}\left(T^{a} T^{b} T^{c} T^{d}\right)|(f ; 0)\rangle \\
& =\frac{1}{N^{3}}\left(N^{2}-4\right)^{2}\left(N^{2}-1\right)|(f ; 0)\rangle \rightarrow N^{3}|(f ; 0)\rangle \text {, } \\
& d^{a b e} d^{c d e}\left(J^{a} K^{b} K^{c} K^{d}\right)_{0}|(f ; 0)\rangle \quad-\frac{1}{N} d^{a b e} d^{c d e} \operatorname{Tr}\left(T^{a} T^{b} T^{c} T^{d}\right)|(f ; 0)\rangle \\
& =-\frac{1}{N^{3}}\left(N^{2}-4\right)^{2}\left(N^{2}-1\right)|(f ; 0)\rangle \rightarrow-N^{3}|(f ; 0)\rangle, \\
& d^{a b e} d^{c d e}\left(K^{a} K^{b} K^{c} K^{d}\right)_{0}|(f ; 0)\rangle=\frac{1}{N} d^{a b e} d^{c d e} \operatorname{Tr}\left(T^{a} T^{b} T^{c} T^{d}\right)|(f ; 0)\rangle \\
& =\frac{1}{N^{3}}\left(N^{2}-4\right)^{2}\left(N^{2}-1\right)|(f ; 0)\rangle \rightarrow N^{3}|(f ; 0)\rangle,
\end{aligned}
$$




$$
\begin{aligned}
\delta^{a b} \delta^{c d}\left(J^{a} J^{b} J^{c} J^{d}\right)_{0}|(f ; 0)\rangle & =\frac{1}{N} \delta^{a b} \delta^{c d} \operatorname{Tr}\left(T^{a} T^{b} T^{c} T^{d}\right)|(f ; 0)\rangle \\
=\frac{1}{N^{2}}\left(N^{2}-1\right)^{2}|(f ; 0)\rangle & \rightarrow N^{2}|(f ; 0)\rangle, \\
\delta^{a b} \delta^{c d}\left(J^{a} J^{b} J^{c} K^{d}\right)_{0}|(f ; 0)\rangle & =-\frac{1}{N} \delta^{a b} \delta^{c d} \operatorname{Tr}\left(T^{a} T^{b} T^{c} T^{d}\right)|(f ; 0)\rangle \\
=-\frac{1}{N^{2}}\left(N^{2}-1\right)^{2}|(f ; 0)\rangle & \rightarrow-N^{2}|(f ; 0)\rangle, \\
\delta^{a b} \delta^{c d}\left(J^{a} J^{b} K^{c} K^{d}\right)_{0}|(f ; 0)\rangle & =\frac{1}{N} \delta^{a b} \delta^{c d} \operatorname{Tr}\left(T^{a} T^{b} T^{c} T^{d}\right)|(f ; 0)\rangle \\
=\frac{1}{N^{2}}\left(N^{2}-1\right)^{2}|(f ; 0)\rangle & \rightarrow N^{2}|(f ; 0)\rangle, \\
\delta^{a b} \delta^{c d}\left(J^{a} K^{b} K^{c} K^{d}\right)_{0}|(f ; 0)\rangle & =-\frac{1}{N} \delta^{a b} \delta^{c d} \operatorname{Tr}\left(T^{a} T^{b} T^{c} T^{d}\right)|(f ; 0)\rangle \\
=-\frac{1}{N^{2}}\left(N^{2}-1\right)^{2}|(f ; 0)\rangle & \rightarrow-N^{2}|(f ; 0)\rangle, \\
\delta^{a b} \delta^{c d}\left(K^{a} K^{b} K^{c} K^{d}\right)_{0}|(f ; 0)\rangle & =\frac{1}{N} \delta^{a b} \delta^{c d} \operatorname{Tr}\left(T^{a} T^{b} T^{c} T^{d}\right)|(f ; 0)\rangle \\
=\frac{1}{N^{2}}\left(N^{2}-1\right)^{2}|(f ; 0)\rangle & \rightarrow N^{2}|(f ; 0)\rangle, \\
\left(\partial^{2} J^{a} K^{a}\right)_{0}|(f ; 0)\rangle & =-\frac{2}{N} \delta^{a b} \operatorname{Tr}\left(T^{a} T^{b}\right)|(f ; 0)\rangle=\frac{2}{N}\left(N^{2}-1\right)|(f ; 0)\rangle \\
& \rightarrow 2 N|(f ; 0)\rangle, \\
\left(\partial J^{a} \partial K^{a}\right)_{0}|(f ; 0)\rangle & =-\frac{1}{N} \delta^{a b} \operatorname{Tr}\left(T^{a} T^{b}\right)|(f ; 0)\rangle=\frac{1}{N}\left(N^{2}-1\right)|(f ; 0)\rangle \\
& \rightarrow N|(f ; 0)\rangle, \\
\left(J^{a} \partial^{2} K^{a}\right)_{0}|(f ; 0)\rangle & =-\frac{2}{N} \delta^{a b} \operatorname{Tr}\left(T^{a} T^{b}\right)|(f ; 0)\rangle=\frac{2}{N}\left(N^{2}-1\right)|(f ; 0)\rangle \\
& \rightarrow 2 N|(f ; 0)\rangle, \\
\delta^{a c} \delta^{b d}\left(J^{a} J^{b} K^{c} K^{d}\right)_{0}|(f ; 0)\rangle & =\frac{1}{N} \delta^{a b} \delta^{c d} \operatorname{Tr}\left(T^{a} T^{b} T^{c} T^{d}\right)|(f ; 0)\rangle \\
\frac{1}{N^{2}}\left(N^{2}-1\right)^{2}|(f ; 0)\rangle & \rightarrow N^{2}|(f ; 0)\rangle . \\
&
\end{aligned}
$$

In particular, the following identity with (A.3) can be used to simplify (F.1)-(F.5):

$$
\begin{aligned}
d^{a b c d} \operatorname{Tr}\left(T^{a} T^{b} T^{c} T^{d}\right)= & 3 d^{a b e} d^{c d e} \operatorname{Tr}\left(T^{a} T^{b} T^{c} T^{d}\right)-\frac{12\left(N^{2}-4\right)}{N\left(N^{2}+1\right)} \delta^{a b} \delta^{c d} \operatorname{Tr}\left(T^{a} T^{b} T^{c} T^{d}\right) \\
& +\left(-\frac{3\left(N^{2}-4\right)\left(N^{2}-3\right)}{N^{2}+1}+\frac{4\left(N^{2}-4\right)\left(N^{2}-3\right)}{N^{2}+1}\right) \delta^{a b} \operatorname{Tr}\left(T^{a} T^{b}\right) \\
= & \frac{2\left(N^{2}-1\right)\left(N^{2}-4\right)\left(N^{2}-9\right)}{N^{2}+1}
\end{aligned}
$$

where the quartic product of the generators with (4.2) has the following identities

$$
\begin{aligned}
d^{a b e} d^{c d e} \operatorname{Tr}\left(T^{a} T^{b} T^{c} T^{d}\right) & =\frac{1}{N^{2}}\left(N^{2}-4\right)^{2}\left(N^{2}-1\right), \\
\delta^{a b} \delta^{c d} \operatorname{Tr}\left(T^{a} T^{b} T^{c} T^{d}\right) & =\delta^{a b} \delta^{c d}\left(\frac{1}{N} \delta^{a b} \delta^{c d}\right)=\frac{1}{N}\left(N^{2}-1\right)^{2} .
\end{aligned}
$$

The following eigenvalue equations for other state can be obtained:

$$
\begin{aligned}
d^{a b c d}\left(J^{a} J^{b} J^{c} J^{d}\right)_{0}|(0 ; f)\rangle & =\frac{1}{N} d^{a b c d} \operatorname{Tr}\left(T^{a} T^{b} T^{c} T^{d}\right)|(0 ; f)\rangle \\
=\frac{2\left(N^{2}-1\right)\left(N^{2}-4\right)\left(N^{2}-9\right)}{N\left(N^{2}+1\right)}|(0 ; f)\rangle & \rightarrow 2 N^{3}|(0 ; f)\rangle,
\end{aligned}
$$




$$
\begin{aligned}
d^{a b e} d^{c d e}\left(J^{a} J^{b} J^{c} J^{d}\right)_{0}|(0 ; f)\rangle & =\frac{1}{N} d^{a b e} d^{c d e} \operatorname{Tr}\left(T^{a} T^{b} T^{c} T^{d}\right)|(0 ; f)\rangle \\
=\frac{1}{N^{3}}\left(N^{2}-4\right)^{2}\left(N^{2}-1\right)|(0 ; f)\rangle & \rightarrow N^{3}|(0 ; f)\rangle, \\
\delta^{a b} \delta^{c d}\left(J^{a} J^{b} J^{c} J^{d}\right)_{0}|(0 ; f)\rangle & =\frac{1}{N} \delta^{a b} \delta^{c d} \operatorname{Tr}\left(T^{a} T^{b} T^{c} T^{d}\right)|(0 ; f)\rangle \\
=\frac{1}{N^{2}}\left(N^{2}-1\right)^{2}|(0 ; f)\rangle & \rightarrow N^{2}|(0 ; f)\rangle .
\end{aligned}
$$

In addition, the identities in (F.20) and (F.21) can be used.

Combine the equations (F.1)-(F.19) with the coefficients in appendix $A$ for the first eigenvalue equation and (F.22), (F.23) and (F.24) with the coefficients for the second eigenvalue equation. The resulting eigenvalue equations have very simple factorized forms as follows:

$$
\begin{aligned}
W_{0}^{(4)}|(f ; 0)\rangle= & {\left[\frac{2\left(N^{2}-1\right)\left(N^{2}-4\right)\left(N^{2}-9\right)}{N\left(N^{2}+1\right)}\left(c_{1}-c_{2}+c_{3}-c_{4}+c_{5}\right)\right.} \\
& +\frac{1}{N^{3}}\left(N^{2}-4\right)^{2}\left(N^{2}-1\right)\left(c_{6}-c_{7}+c_{8}-c_{9}+c_{10}\right) \\
& +\frac{1}{N^{2}}\left(N^{2}-1\right)^{2}\left(c_{11}-c_{12}+c_{13}-c_{14}+c_{15}\right) \\
& \left.+\frac{1}{N}\left(N^{2}-1\right)\left(2 c_{17}+c_{20}+2 c_{22}\right)+\frac{1}{N^{2}}\left(N^{2}-1\right)^{2} c_{23}\right]|(f ; 0)\rangle \\
= & {\left[\frac{2(k+1)(N-3)\left(N^{2}-1\right)(k+2 N)(k+2 N+1)(3 k+4 N+3)}{N^{2}(k+N)(2 k+N) d(N, k)}\right]|(f ; 0)\rangle, } \\
W_{0}^{(4)}|(0 ; f)\rangle= & {\left[\frac{2\left(N^{2}-1\right)\left(N^{2}-4\right)\left(N^{2}-9\right)}{N\left(N^{2}+1\right)} c_{1}+\frac{1}{N^{3}}\left(N^{2}-4\right)^{2}\left(N^{2}-1\right) c_{6}\right.} \\
& \left.+\frac{1}{N^{2}}\left(N^{2}-1\right)^{2} c_{11}\right]|(0 ; f)\rangle \\
= & {\left[\frac{2 k(k+1)(N-3)\left(N^{2}-1\right)(k+2 N)(3 k+2 N)}{N^{2}(k+N+1)(2 k+3 N+2) d(N, k)}\right]|(0 ; f)\rangle, }
\end{aligned}
$$

where $d(N, k) \equiv 17 k+17 k^{2}+22 N+39 k N+5 k^{2} N+22 N^{2}+10 k N^{2}$.

The spin-3 current case can be analyzed further as follows:

$$
\begin{aligned}
W_{0}^{(3)}|(f ; 0)\rangle & =\left(-A_{1}+A_{2}-A_{3}+A_{4}\right) d^{a b c} K_{0}^{a} K_{0}^{b} K_{0}^{c}|(f ; 0)\rangle \\
& =\left(-A_{1}+A_{2}-A_{3}+A_{4}\right)\left[\frac{i}{N^{2}}\left(N^{2}-4\right)\left(N^{2}-1\right)\right]|(f ; 0)\rangle \\
& =-\left[\frac{i}{N^{2}}\left(N^{2}-4\right)\left(N^{2}-1\right)(k+N+1)(k+2 N+1)(2 k+3 N+2)\right]|(f ; 0)\rangle, \\
W_{0}^{(3)}|(0 ; f)\rangle & =A_{1} d^{a b c} J_{0}^{a} J_{0}^{b} J_{0}^{c}|(0 ; f)\rangle=A_{1}\left[\frac{i}{N^{2}}\left(N^{2}-4\right)\left(N^{2}-1\right)\right]|(0 ; f)\rangle \\
& =\left[\frac{i}{N^{2}} k\left(N^{2}-4\right)\left(N^{2}-1\right)(k+N)(2 k+N)\right]|(0 ; f)\rangle,
\end{aligned}
$$

where the property (4.15) is used and the coefficients in (3.8) are substituted. 
Finally, the eigenvalue equations for the spin-2 current can be summarized as

$$
\begin{aligned}
T_{0}|(f ; 0)\rangle= & {\left[-\frac{k}{2(N+1)(k+N+1)} J_{0}^{a} J_{0}^{a}-\frac{1}{2(k+N)(k+N+1)} K_{0}^{a} K_{0}^{a}\right.} \\
& \left.+\frac{1}{k+N+1} J_{0}^{a} K_{0}^{a}\right]|(f ; 0)\rangle \\
= & \left(-\frac{k+2 N+1}{2(N+1)(k+N)}\right)\left[-\frac{1}{N}\left(N^{2}-1\right)\right]|(f ; 0)\rangle \\
= & {\left[\frac{(N-1)(k+2 N+1)}{2 N(k+N)}\right]|(f ; 0)\rangle, } \\
T_{0}|(0 ; f)\rangle= & -\frac{k}{2(N+1)(k+N+1)} J_{0}^{a} J_{0}^{a}|(0 ; f)\rangle \\
= & -\frac{k}{2(N+1)(k+N+1)}\left[-\frac{1}{N}\left(N^{2}-1\right)\right]|(0 ; f)\rangle \\
= & {\left[\frac{k(N-1)}{2 N(k+N+1)}\right]|(0 ; f)\rangle, }
\end{aligned}
$$

where the property $\delta^{a b} \operatorname{Tr}\left(T^{a} T^{b}\right)=-\left(N^{2}-1\right)$ is used.

Open Access. This article is distributed under the terms of the Creative Commons Attribution License (CC-BY 4.0), which permits any use, distribution and reproduction in any medium, provided the original author(s) and source are credited.

\section{References}

[1] M.R. Gaberdiel and R. Gopakumar, An AdS $S_{3}$ dual for minimal model CFTs, Phys. Rev. D 83 (2011) 066007 [arXiv:1011.2986] [InSPIRE].

[2] M.R. Gaberdiel and R. Gopakumar, Triality in minimal model holography, JHEP 07 (2012) 127 [arXiv: 1205.2472] [INSPIRE].

[3] P. Bouwknegt and K. Schoutens, $W$ symmetry in conformal field theory, Phys. Rept. 223 (1993) 183 [hep-th/9210010] [INSPIRE].

[4] F. Bais, P. Bouwknegt, M. Surridge and K. Schoutens, Coset construction for extended Virasoro algebras, Nucl. Phys. B 304 (1988) 371 [INSPIRE].

[5] M.R. Gaberdiel and R. Gopakumar, Minimal model holography, J. Phys. A 46 (2013) 214002 [arXiv:1207.6697] [INSPIRE].

[6] M. Ammon, M. Gutperle, P. Kraus and E. Perlmutter, Black holes in three dimensional higher spin gravity: a review, J. Phys. A 46 (2013) 214001 [arXiv:1208.5182] [INSPIRE].

[7] C. Ahn, The coset Spin-4 Casimir operator and its three-point functions with scalars, JHEP 02 (2012) 027 [arXiv: 1111.0091] [INSPIRE].

[8] P. Bowcock, Quasi-primary fields and associativity of chiral algebras, Nucl. Phys. B 356 (1991) 367 [INSPIRE].

[9] R. Blumenhagen et al., $W$ algebras with two and three generators, Nucl. Phys. B 361 (1991) 255 [inSPIRE]. 
[10] W. Nahm, Algebras of two-dimensional chiral fields and their classification, in Proceedings, mathematical physics, Islamabad Pakistan (1989), pg. 283 [INSPIRE].

[11] W. Nahm, Chiral algebras of two-dimensional chiral field theories and their normal ordered products, in Proceedings, recent developments in conformal field theories, Trieste Italy (1989), pg. 81 [INSPIRE].

[12] C. Ahn, The higher spin currents in the $N=1$ stringy coset minimal model, JHEP 04 (2013) 033 [arXiv:1211.2589] [INSPIRE].

[13] M.R. Gaberdiel and T. Hartman, Symmetries of holographic minimal models, JHEP 05 (2011) 031 [arXiv:1101.2910] [INSPIRE].

[14] H. Kausch and G. Watts, A study of $W$ algebras using Jacobi identities, Nucl. Phys. B 354 (1991) 740 [inSPIRE].

[15] H. Ozer, On the construction of $W_{N}$ algebras in the form of $A_{N-1}$ Casimir algebras, Mod. Phys. Lett. A 11 (1996) 1139 [hep-th/9609120] [INSPIRE].

[16] K. Hornfeck, The minimal supersymmetric extension of $W A_{N-1}$, Phys. Lett. B 275 (1992) 355 [inSPIRE].

[17] K. Thielemans, A Mathematica package for computing operator product expansions, Int. J. Mod. Phys. C 2 (1991) 787 [inSPIRE].

[18] C.-M. Chang and X. Yin, Higher spin gravity with matter in $A d S_{3}$ and its CFT dual, JHEP 10 (2012) 024 [arXiv:1106.2580] [INSPIRE].

[19] M. Ammon, P. Kraus and E. Perlmutter, Scalar fields and three-point functions in $D=3$ higher spin gravity, JHEP 07 (2012) 113 [arXiv:1111.3926] [INSPIRE].

[20] R. Blumenhagen, W. Eholzer, A. Honecker, K. Hornfeck and R. Hubel, Coset realization of unifying $W$ algebras, Int. J. Mod. Phys. A 10 (1995) 2367 [hep-th/9406203] [INSPIRE].

[21] J. de Azcarraga, A. Macfarlane, A. Mountain and J. Perez Bueno, Invariant tensors for simple groups, Nucl. Phys. B 510 (1998) 657 [physics/9706006] [INSPIRE].

[22] M.R. Gaberdiel, T. Hartman and K. Jin, Higher spin black holes from CFT, JHEP 04 (2012) 103 [arXiv: 1203.0015] [INSPIRE].

[23] K. Hornfeck, $W$ algebras with set of primary fields of dimensions $(3,4,5)$ and $(3,4,5,6)$, Nucl. Phys. B 407 (1993) 237 [hep-th/9212104] [INSPIRE].

[24] C. Ahn, Higher spin currents with arbitrary $N$ in the $\mathcal{N}=\infty$ stringy coset minimal model, JHEP 07 (2013) 141 [arXiv:1305.5892] [INSPIRE].

[25] C.-H. Ahn, K. Schoutens and A. Sevrin, The full structure of the super $W_{3}$ algebra, Int. J. Mod. Phys. A 6 (1991) 3467 [inSPIRE].

[26] C. Ahn, The large- $N$ 't Hooft limit of coset minimal models, JHEP 10 (2011) 125 [arXiv:1106.0351] [INSPIRE].

[27] M.R. Gaberdiel and C. Vollenweider, Minimal model holography for $\mathrm{SO}(2 \mathrm{~N})$, JHEP 08 (2011) 104 [arXiv:1106.2634] [INSPIRE].

[28] C. Ahn, The primary Spin-4 Casimir operators in the holographic $\mathrm{SO}(N)$ coset minimal models, JHEP 05 (2012) 040 [arXiv:1202.0074] [INSPIRE].

[29] C. Ahn and J. Paeng, The OPEs of Spin-4 Casimir currents in the holographic $\mathrm{SO}(N)$ coset minimal models, Class. Quant. Grav. 30 (2013) 175004 [arXiv:1301.0208] [INSPIRE]. 
[30] M.R. Gaberdiel and R. Gopakumar, Large $\mathcal{N}=4$ holography, JHEP 09 (2013) 036 [arXiv: 1305.4181] [INSPIRE].

[31] C. Ahn, The large-N 't Hooft limit of Kazama-Suzuki model, JHEP 08 (2012) 047 [arXiv: 1206.0054] [INSPIRE].

[32] C. Ahn, The operator product expansion of the lowest higher spin current at finite $N$, JHEP 01 (2013) 041 [arXiv: 1208.0058] [INSPIRE].

[33] K. Schoutens, Yangian symmetry in conformal field theory, Phys. Lett. B 331 (1994) 335 [hep-th/9401154] [INSPIRE].

[34] F. Bais, P. Bouwknegt, M. Surridge and K. Schoutens, Extensions of the Virasoro algebra constructed from Kac-Moody algebras using higher order Casimir invariants, Nucl. Phys. B 304 (1988) 348 [InSPIRE]. 